University of Nebraska - Lincoln

DigitalCommons@University of Nebraska - Lincoln

October 2007

\title{
1,3-Alternate calix[4]arene nitronyl nitroxide tetraradical and diradical: synthesis, X-ray crystallography, paramagnetic NMR spectroscopy, EPR spectroscopy, and magnetic studies
}

\author{
Andrzej Rajca \\ University of Nebraska - Lincoln, arajca1@unl.edu \\ Maren Pink \\ Indiana University \\ Sumit Mukherjee \\ University of Nebraska - Lincoln \\ Suchada Rajca \\ University of Nebraska-Lincoln, srajca1@unl.edu \\ Kausik Das \\ University of Nebraska - Lincoln
}

Follow this and additional works at: https://digitalcommons.unl.edu/chemistryrajca

Part of the Chemistry Commons

Rajca, Andrzej; Pink, Maren; Mukherjee, Sumit; Rajca, Suchada; and Das, Kausik, "1,3-Alternate calix[4]arene nitronyl nitroxide tetraradical and diradical: synthesis, X-ray crystallography, paramagnetic NMR spectroscopy, EPR spectroscopy, and magnetic studies" (2007). Andrzej Rajca Publications. 2. https://digitalcommons.unl.edu/chemistryrajca/2

This Article is brought to you for free and open access by the Published Research - Department of Chemistry at DigitalCommons@University of Nebraska - Lincoln. It has been accepted for inclusion in Andrzej Rajca Publications by an authorized administrator of DigitalCommons@University of Nebraska - Lincoln. 


\title{
1,3-Alternate calix[4]arene nitronyl nitroxide tetraradical and diradical: synthesis, X-ray crystallography, paramagnetic NMR spectroscopy, EPR spectroscopy, and magnetic studies
}

\author{
Andrzej Rajca*, Maren Pink ${ }^{\dagger}$, Sumit Mukherjee*, Suchada Rajca*, and Kausik Das* \\ * Department of Chemistry, University of Nebraska-Lincoln, Lincoln, NE 68588-0304, USA \\ ${ }^{\dagger}$ Department of Chemistry, Indiana University, Bloomington, IN 47405-7102, USA \\ $¥$ Corresponding author. Tel.: +1 402472 9196; fax: +1 4024729402
}

\begin{abstract}
Calix[4]arenes constrained to 1,3-alternate conformation and functionalized at the upper rim with four and two nitronyl nitroxides have been synthesized, and characterized by X-ray crystallography, magnetic resonance (EPR and ${ }^{1} \mathrm{H}$ NMR) spectroscopy, and magnetic studies. Such calix[4]arene tetraradicals and diradicals provide scaffolds for throughbond and through-space intramolecular exchange couplings.
\end{abstract}

Keywords: Calixarene, Nitroxide, Diradical, Tetraradical, Magnetic properties, Paramagnetic NMR spectroscopy

\section{Introduction}

Macrocycles based upon calix[4]arenes have been utilized as effective frameworks for high-spin polyradicals. ${ }^{[1]},[2],[3], \&$ [4] In such macrocycles, four triphenylmethyl radicals are connected in-chain, forming cyclic cross-conjugated $\pi$-systems, in which the radicals are coupled through $m$-phenylene, a wellknown, robust through-bond ferromagnetic exchange coupler (Figure 1). ["], [6], [7], [8], [9], [10], \& [11]

The tetraradical possesses a high-spin quintet $(S=2)$ ground state, with a large energy gap separating the $S=2$ ground state from the $S<2$ excited states. ${ }^{12}$ The polyarylmethyl calix[4]arene $S=2$ tetraradical (Figure 1) is the key building block for the bottom-up, modular design of high-spin organic polyradicals. $^{[1], ~[2], ~[3], ~[4], ~[13], ~[14], ~[15], ~[16], ~[17], ~[18], ~ \& ~[19] ~ E x a m-~}$ ples of such polyradicals include organic molecules with the highest values of the total spin quantum number up to $S=13$ and the first organic polymer with magnetic ordering (magnetic ordering temperature of about $10 \mathrm{~K}){ }^{[16] \&[17]}$

Recently, calix[4]arene and resorcinarene macrocycles have been adopted as scaffolds for attaching two or four organic radicals as pendants at the upper or lower rim. [20], [21], [21a], [21b], [22], [23], \& [24] For example, direct attachment of radicals at the upper rim of calix[4]arenes that are constrained to an 1,3-alternate or cone conformation has provided unique models for the dissection of the through-bond and through-space intramolecular exchange coupling, as well as for the study of conformational dependence of through-bond coupling (Figure 2). ${ }^{[23] ~ \& ~}$ ${ }^{[24]}$ The through-bond coupling between the adjacent radicals is mediated by the relatively extended radical $-m$-phenylene$\mathrm{CH}_{2}-m$-phenylene-radical coupling pathway, and throughspace coupling is found between the diagonal radicals.
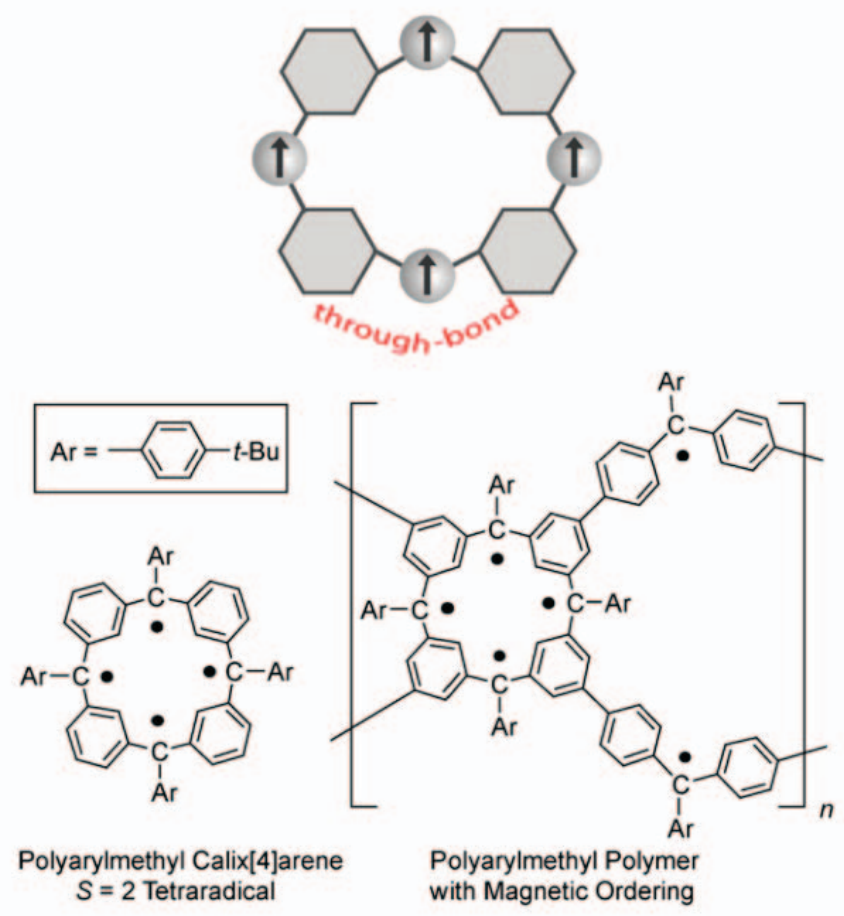

Figure 1. Calix[4]arenes functionalized with in-chain radicals forming a cross-conjugated $\pi$-system: polyarylmethyl $S=2$ tetraradical and polymer with magnetic ordering. 
Magnetic studies of ambient stable nitroxide tetraradicals (Figure 2) indicate that the through-bond exchange coupling, mediated by the nitroxide- $m$-phenylene- $\mathrm{CH}_{2}-m$-phenylenenitroxide exchange coupling pathway, is antiferromagnetic in the 1,3-alternate conformation, while the exchange coupling is ferromagnetic in the cone conformation. ${ }^{[24]}$ Through-space nitroxide-nitroxide exchange couplings are antiferromagnetic in both cone and 1,3-alternate calix[4]arene tetraradical, as well as in the 1,3-alternate calix[4]arene diradical. X-ray structures of the diradical and tetraradical reveal $\mathrm{N} \cdots \mathrm{N}$ distances of 5$6 \AA$ between the diagonal nitroxides. Both through-bond and through-space exchange couplings are on the order of $1 \mathrm{~K}$, i.e., $|J / k| \approx 1 \mathrm{~K} .{ }^{[24]}$

The radical functionalized 1,3-alternate calix[4]arene is an attractive model system with well-defined radical-radical interactions, such as exchange coupling and magnetic dipoledipole coupling, for probing the dependence of electron spin relaxation on the strength of radical-radical interactions. Recently, the 1,3-alternate calix[4]arene nitroxide tetraradical and diradical were employed as model systems for the study of electron spin relaxation, ${ }^{[25]}$ aimed at the development of functional paramagnetic contrast agents for electron paramagnetic resonance imaging (EPRI) and magnetic resonance imaging (MRI). [26], [27], [28], [29], \& [30] These nitroxide polyradicals provide the probes for well-defined and moderately strong radical-radical interactions.

We have sought model systems, in which we anticipate significantly weaker radical-radical interactions, such as nitronyl nitroxide tetraradical 1 and diradical 2 (Figure 3). ${ }^{[31] ~ \& ~[32] ~}$

The analysis of the EPR data in the literature indicates that the ${ }^{1} \mathrm{H}$-hyperfine splittings $\left(a_{\mathrm{H}}\right)$ at the para- and ortho-positions in 2-phenyl-4,4,5,5-tetramethylimidazoline-1-oxyl-3-oxide (phenyl nitronyl nitroxide) monoradical are about $1 / 5$, compared

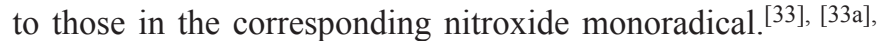
[33b], [34], [34a], \& [34b] Based upon the empirical relationship (2J/ $k \sim\left|a_{\mathrm{H}}\right|^{2},{ }^{[2]}$ which reflects the correlation between the strength of through-bond exchange coupling mediated by $m$-phenylene and the delocalization of spin density into the $m$-phenylene, the singlet-triplet gap, $2 J / k$, for $m$-phenylene-based nitronyl nitroxide diradicals should be smaller by a factor of $1 / 25$ than that for the corresponding nitroxide diradicals. This estimate is supported by the experimental values of the singlettriplet gaps in $m$-phenylene-based diradicals that are $2 J / k \approx$ $+600 \mathrm{~K}$ and $+20 \mathrm{~K}$ for bis(nitroxide) and bis(nitronyl nitroxide), respectively. ${ }^{[30], ~[35], ~ \& ~[36] ~ T h e r e f o r e, ~ t h e ~ t h r o u g h-b o n d ~}$ exchange coupling mediated by the $m$-phenylene- $\mathrm{CH}_{2}-m$ phenylene unit in nitronyl nitroxide tetraradical $\mathbf{1}$ is expected to be weaker by a factor of $25-30$, compared to $|J / k| \approx 1 \mathrm{~K}$ in the corresponding nitroxide tetraradical.

Through-space radical-radical interactions depend on the radical-radical distance $(r)$, with exponential and $1 / r^{3}$ dependencies for through-space exchange coupling and magnetic dipole-dipole coupling, respectively. Because the 4,4,5,5-tet- ramethylimidazoline-1-oxyl-3-oxide (nitronyl nitroxide) moieties are more bulky compared to the tert-butylnitroxide moieties, the distances between co-facial nitronyl nitroxides in tetraradical $\mathbf{1}$ and in diradical $\mathbf{2}$ are expected to be greater than those in the corresponding nitroxide tetraradical and diradical. In addition, the spin density is primarily delocalized over four atoms $\left(\mathrm{N}_{2} \mathrm{O}_{2}\right)$ in the nitronyl nitroxide moieties, compared to the delocalization over two atoms (NO) in the nitroxide moieties. These increased radical-radical distances and increased delocalization of spin density should lead to weaker throughspace radical-radical interactions between co-facial nitronyl nitroxides in $\mathbf{1}$ and $\mathbf{2}$.

This article describes the synthesis and characterization of ambient stable nitronyl nitroxide tetraradical $\mathbf{1}$ and diradical $\mathbf{2}$ in the fixed 1,3-alternate calix[4]arene conformations (Figure 3).
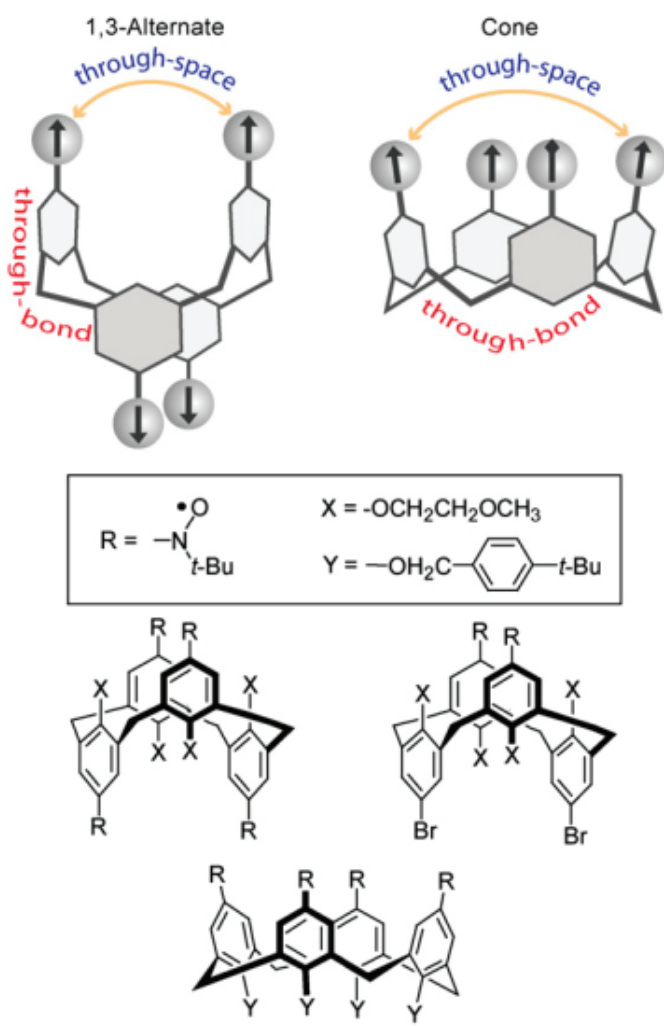

Figure 2. Calix[4]arenes functionalized with pendant radicals: 1,3-alternate and cone calix[4]arene scaffolds with two or four nitroxide radicals.

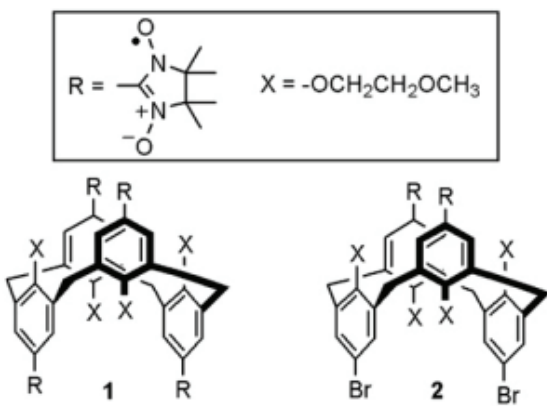

Figure 3. Nitronyl nitroxide tetraradical 1 and diradical $\mathbf{2}$ with constrained conformations of 1,3-alternate calix[4]arene. 


\section{Results and discussion}

\subsection{Synthesis}

The synthesis starts from tetrabromocalix[4]arene $\mathbf{3}$, constrained in the 1,3-alternate conformation (Scheme 1). ${ }^{[37]}$ \& [24] $\mathrm{Li} / \mathrm{Br}$ exchange on 3 with an excess amount of $t$-BuLi gives intermediate tetrakis(aryllithium), which is reacted with $\mathrm{N}$ methylformanilide, to provide tetraaldehyde $4 .{ }^{[38]}$

An analogous procedure, in which the $t$-BuLi is replaced with $n$-BuLi (2 equiv), ${ }^{[24]}$ gives dialdehyde 5. In the next step, well-stirred suspensions of $\mathbf{4}$ and $\mathbf{5}$ in methanol are condensed with 2,3-bis(hydroxyamino)-2,3-dimethylbutane in the presence of $p$-TsOH, ${ }^{[39]}$ to provide tetrakis(1,3-dihydroimidazolidine) 6 and bis(1,3-dihydroimidazolidine) 7, respectively. Oxidations of 6 and 7 in water/dichloromethane at $0{ }^{\circ} \mathrm{C}$, using 1 equiv of $\mathrm{NaIO}_{4}$ per 1,3-dihydroimidazolidine, ${ }^{[39]}$ give tetraradical 1 and diradical $\mathbf{2}$ in good yields.

The presence of hydroxyl groups in $\mathbf{6}$ and $\mathbf{7}$ is confirmed by IR (e.g., $v_{\mathrm{O}-\mathrm{H}} \approx 3300 \mathrm{~cm}^{-1}$ ) and ${ }^{1} \mathrm{H}$ NMR (600 MHz, DMSO$d_{6}$ ) spectra; in particular, $\mathrm{D}_{2} \mathrm{O}$-exchangeable 8-proton or 4proton singlets at 7.6-7.7 ppm are found in the ${ }^{1} \mathrm{H}$ NMR spectra. The additional evidence for 1,3-dihydroimidazolidines is provided by the assignment of the methine protons (4-proton and 2-proton singlets at 4.4-4.5 ppm), which are slightly upfield shifted from the typical 4.6-4.8 ppm range; the methyl groups on the imidazolidine rings appear as two singlets at $\sim 1.0 \mathrm{ppm}$ for each compound. ${ }^{13} \mathrm{C}$ NMR $(150 \mathrm{MHz}$, DMSO$d_{6}$ ) spectra for $\mathbf{6}$ and $\mathbf{7}$ show all expected resonances in the aromatic and aliphatic regions, including the resonances for methine carbons of the imidazolidine rings at $89-90 \mathrm{ppm}$.

\subsection{Molecular structure of 1 and 2}

Nitronyl nitroxide tetraradical $\mathbf{1}^{[40]}$ and diradical 2 (Ref. S5, Supplementary data) crystallize with two unique molecules per asymmetric unit and include solvent in the structures. Ineffi- cient packing of the calix[4]arenes leaves voids in the structure. This, together with the inherent flexibility of the methoxyethylene chains and the rotational freedom of the imidazole rings, gives rise to extensive disorder. In fact, the diffraction pattern indicated highly disordered structures by streaks, diffuse (non-Bragg) scattering, and rapidly weakening diffraction at higher $(\sin \theta) / \lambda$ values (at medium and high resolution). Consequently, the resulting structures have relatively high $R$ values. However, the standard uncertainties of geometrical parameters are reasonable for $\mathbf{1}$ and the disorder is affecting solvent molecules, side chains and the imidazoline rings (rotationally disordered about the bond to the calixarene frame) only but not the calixarene core. The displacement parameters (Figure 4) are acceptable as well. We therefore assert that the X-ray structure of 1 (Figure 4 and Figure S1, Supplementary data) provides correct connectivity and reliable geometry, and distances discussed (vide infra) are not adversely affected by the low quality of the entire structure with disorder in side chains and solvent molecules. Moreover with two independent molecules, we have several observations for distances and angles discussed, which fit quite well and are within the expected ranges. However, 2 should be viewed as a preliminary result that confirms the connectivity and overall geometry of the diradical only (Figures S2 and S3, Supplementary data).

The structure of tetraradical $\mathbf{1}$ has four pairs of the co-facial nitronyl nitroxide groups (ONCNO), due to the presence of two crystallographically unique molecules. The disorder of two ONCNO groups, with the 50:50 site occupancy in each case, is associated with the rotation of the 4,4,5,5-tetramethylimidazoline-1-oxyl-3-oxide moiety about the CC bond connecting it to the benzene ring. For each aryl nitronyl nitroxide moiety, the ONCNO groups are twisted about this CC bond, forming torsional angles of $10-40^{\circ}$ with the benzene rings. In most pairs of co-facial radicals, the ONCNO groups are nearly parallel; the radical-radical distances are rather long, as measured by the $\mathrm{C} \cdots \mathrm{C}$ distances that are between 6.68 and $7.13 \AA$ for all four pairs (Table S1, Supplementary data). ${ }^{[11 a]}$

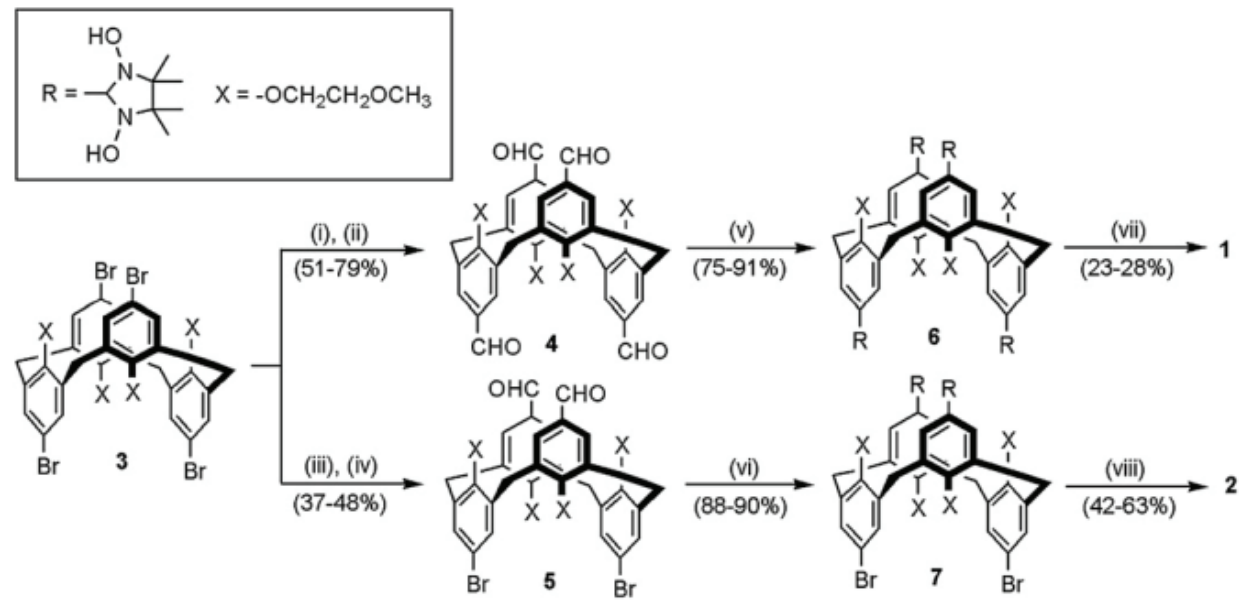

Scheme 1. Synthesis of nitronyl nitroxide tetraradical 1 and diradical 2. Reagents and conditions: (i) $t$-BuLi ( 8 equiv), THF, $-78^{\circ} \mathrm{C}$ for $2 \mathrm{~h}$, then $-20{ }^{\circ} \mathrm{C}$ for $15 \mathrm{~min}$; (ii) $\mathrm{PhN}(\mathrm{Me}) \mathrm{CHO}$ (4 equiv), $-78^{\circ} \mathrm{C}$ then slowly warm up to room temperature overnight; (iii) $n$ - $\mathrm{BuLi}\left(2\right.$ equiv), $\mathrm{THF},-78^{\circ} \mathrm{C}$ for $2 \mathrm{~h}$, then $-20^{\circ} \mathrm{C}$ for $15 \mathrm{~min}$; (iv) $\mathrm{PhN}(\mathrm{Me}) \mathrm{CHO}$ (2.4 equiv), $-78^{\circ} \mathrm{C}$ then slowly warm up to room temperature overnight; (v) 2,3-bis(hydroxyamino)-2,3-dimethylbutane (10 equiv), $p$ - TsOH ( 0.2 equiv), $\mathrm{MeOH}, 60-65^{\circ} \mathrm{C}$ for $2-2.5$ days; (vi) 2,3-bis(hydroxyamino)-2,3-dimethylbutane ( 5 equiv), $p$ - $\mathrm{TsOH}\left(0.2\right.$ equiv), $\mathrm{MeOH}, 55-60{ }^{\circ} \mathrm{C}$ for $2-3$ days; (vii) $\mathrm{NaIO}_{4}$ (4 equiv), $\mathrm{CH}_{2} \mathrm{Cl}_{2}, \mathrm{H}_{2} \mathrm{O}, 0^{\circ} \mathrm{C}$; (viii) $\mathrm{NaIO}_{4}$ (2 equiv), $\mathrm{CH}_{2} \mathrm{Cl}_{2}, \mathrm{H}_{2} \mathrm{O}, 0{ }^{\circ} \mathrm{C}$. 


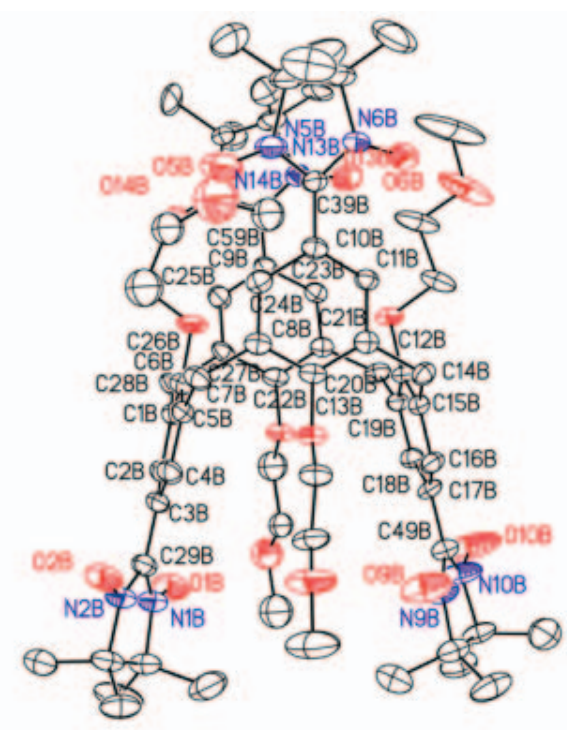

Figure 4. Molecular structure and conformation for nitronyl nitroxide tetraradical 1 with constrained conformation of 1,3-alternate calix[4]arene. Carbon, nitrogen, and oxygen atoms are depicted with thermal ellipsoids and spheres set at the $50 \%$ probability level. Only one of the two unique molecules of 1, omitting disorder, hydrogen atoms, and the solvent of crystallization (benzene and water), is shown.

In diradical 2, the structures for the two crystallographically unique molecules show the two nitronyl nitroxide (ONCNO) groups even further apart, with $\mathrm{C} \cdots \mathrm{C}$ distances of approximately $7.7 \AA,{ }^{[41 b]}$ compared to $\mathbf{1}$ (Table S1, Supplementary data). In each unique molecule, the ONCNO groups are nearly parallel and they form torsional angles of $\sim 30^{\circ}$ with the benzene rings within each aryl nitronyl nitroxide moiety.

In both $\mathbf{1}$ and $\mathbf{2}$, the $\mathrm{C} \cdots \mathrm{C}$ distances of 7-8 $\AA$ between the cofacial ONCNO groups are considerably longer than the corresponding $\mathrm{N} \cdots \mathrm{N}$ distances of 5-6 $\AA$ between the co-facial NO groups in the corresponding nitroxide tetraradical and diradical. These increased distances, especially in diradical $\mathbf{2}$, are associated with the larger dihedral angles between the co-facial benzene rings that are in the $20-25^{\circ}$ range in 1 , in the $36-39^{\circ}$ range in 2 , and in the $2.9-14.0^{\circ}$ range in the corresponding nitroxide tetraradical and diradical (Table S2, Supplementary data). ${ }^{[41 \mathrm{c}]}$ This behavior may be caused by the larger steric repulsion of the 4,4,5,5-tetramethylimidazoline-1-oxyl-3-oxide moieties, compared to the tert-butylnitroxide moieties at the upper rim of the 1,3-alternate calix[4]arene. Most importantly, the increased radical-radical distances should contribute to the relatively weaker through-space radical-radical interactions in $\mathbf{1}$ and $\mathbf{2}$.

\subsection{Structure of 1 and 2 in solution}

${ }^{1} \mathrm{H}$ NMR spectra of tetraradical $\mathbf{1}$ and diradical $\mathbf{2}$ in chloroform- $d$ at room temperature show the expected resonances (Figure 5, Figures S4 and S5, Supplementary data). All ${ }^{1} \mathrm{H}$ resonances should be detectable, especially in concentrated solutions, because of the low-to-moderate spin densities at all of the hydrogen atoms in $\mathbf{1}$ and 2. ${ }^{[2]} \&$ [42] The reported chemical shifts are not corrected for bulk susceptibility shifts. ${ }^{[43], ~[43 a] ~ \& ~[43 b] ~}$

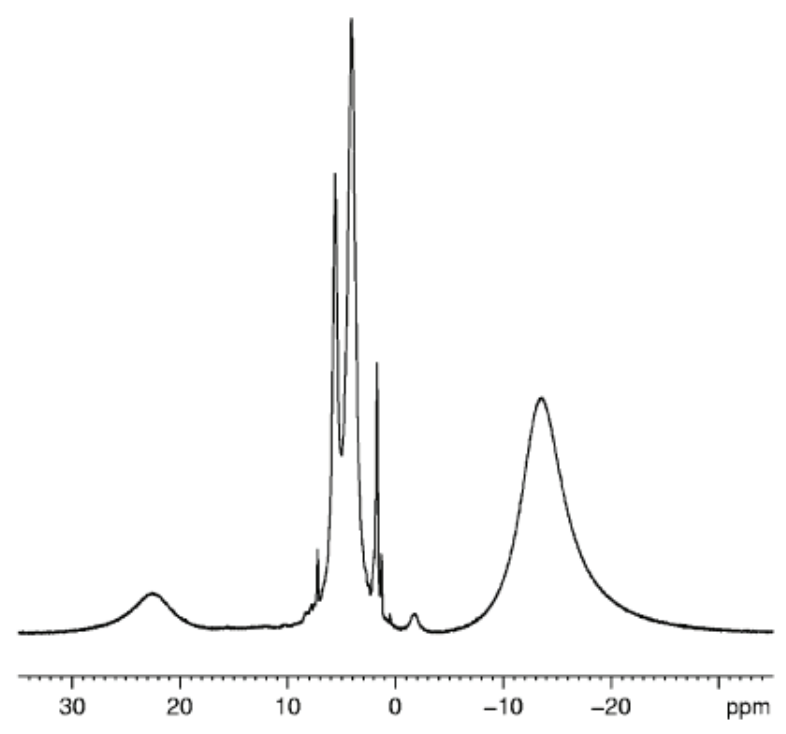

Figure 5. ${ }^{1} \mathrm{H}$ NMR $(600 \mathrm{MHz}, \mathrm{LB}=1 \mathrm{~Hz})$ spectrum for $\sim 70 \mathrm{mM}$ nitronyl nitroxide tetraradical 1 in chloroform- $d$. The weak sharp singlet at $7.26 \mathrm{ppm}$ and broad singlet at $-1.7 \mathrm{ppm}$ correspond to the residual non-deuterated chloroform and water peaks. The chemical shift ( +1 to $-2 \mathrm{ppm})$ and integration of the broad singlet are sample (concentration) dependent. The other sharp, relatively weak peaks are assigned to diamagnetic impurities.

Four broad resonances are observed for $\sim 70 \mathrm{mM}$ tetraradical 1 (Figure 5). The relatively less broadened 12-proton and 24-proton singlets at +5.6 and $+4.1 \mathrm{ppm}$ are assigned to the methyl $\left(\mathrm{CH}_{3}\right)$ and dimethylene $\left(\mathrm{CH}_{2} \mathrm{CH}_{2}\right)$ of the methoxyethyleneoxy $\left(\mathrm{OCH}_{2} \mathrm{CH}_{2} \mathrm{OCH}_{3}\right)$ groups, respectively; in addition, the $+4.1 \mathrm{ppm}$ singlet is assumed to consist of overlapping resonances from the methylene groups of the macrocycle. The two very broad resonances, 8 -proton at $+22.5 \mathrm{ppm}$ and 48 -proton at $-13.6 \mathrm{ppm}$, are assigned to the ortho-hydrogens of the benzene rings and the methyl groups of the oxazoline moieties, respectively. Overall, the spectrum provides evidence for a tetraradical with a $D_{2 \mathrm{~d}}$ point group on the NMR time scale.

Five broad resonances, with similar chemical shifts to those in $\mathbf{1}$, may be identified for diradical $\mathbf{2}$. These resonances may be assigned to the aryl nitronyl nitroxide moieties, as well as to four methylene groups of the macrocycle. ${ }^{[44]}$ In addition, relatively narrow singlets at $7.28,3.41$, and $3.21 \mathrm{ppm}$ are assigned to the aromatic, $\mathrm{OCH}_{3}$, and $\mathrm{OCH}_{2} \mathrm{CH}_{2} \mathrm{O}$ protons of the diamagnetic bromophenyl moieties of $\mathbf{2}$, respectively.

The ${ }^{1} \mathrm{H}$ NMR spectral assignments for aryl nitronyl nitroxide moieties in $\mathbf{1}$ and $\mathbf{2}$ are similar to those for the corresponding nitronyl nitroxide monoradicals, e.g., 2-phenyl-4,4,5,5-tetramethylimidazoline-1-oxyl-3-oxide, thus confirming the structure of the tetraradical and diradical in solution (Figures S17 and S18, Supplementary data). ${ }^{[33 a]}$ However, the chemical shifts for $\mathbf{1}$ and $\mathbf{2}$ are significantly different when compared to the corresponding calix[4]arene nitroxide tetraradical and diradical. ${ }^{[24]}$ Notably, the chemical shifts for the methylene groups of the macrocycle, are about $+4 \mathrm{ppm}$ in 1, compared to $-1 \mathrm{ppm}$ in the corresponding nitroxide tetraradical. ${ }^{[2]}$ This small paramagnetic shift in $\mathbf{1}$ indicates that the spin density at the hydrogens of the methylene groups in $\mathbf{1}$ is very small; in the corresponding nitroxide tetraradical the spin density was 
relatively large and negative, based upon a large upfield paramagnetic shift.

EPR spectra of tetraradical 1 and diradical $\mathbf{2}$ in toluene/chloroform (4:1) at $296 \mathrm{~K}$ show resolved multiplets corresponding to ${ }^{14} \mathrm{~N}$-hyperfine splitting from eight and four nitrogens, respectively (Figure 6). Adequate simulations of the spectra using a single component, e.g., nitronyl nitroxide tetraradical for $\mathbf{1}$, are obtained, confirming high degree of radical purity $(99 \%$ or higher) of 1 and $2 .{ }^{[45]}$ The simulation for the diradical is of somewhat lower quality, presumably due to incomplete motional averaging of dipolar couplings. This is consistent with the significantly greater linewidth in the spectra of $\mathbf{2}$ in solution and greater spectral width for diradical 2 in a rigid matrix, compared to those for tetraradical 1 (Figure 6 and Figure 7). The ${ }^{14} \mathrm{~N}$-hyperfine couplings lead to peak-to-peak splitting $\Delta H_{\mathrm{pp}} \approx 0.188 \mathrm{mT}=a_{\mathrm{N}} / 4$ and $\Delta H_{\mathrm{pp}} \approx 0.376 \mathrm{mT}=a_{\mathrm{N}} / 2$ for $\mathbf{1}$ and 2, respectively; this corresponds to an identical value of $\left|a_{\mathrm{N}}\right|=0.752 \mathrm{mT}$ for $\mathbf{1}$ and $\mathbf{2}$, which is similar to the value of $\left|a_{\mathrm{N}}\right|=0.753 \mathrm{mT}$ reported for the 4-hydroxyphenyl-substituted nitronyl nitroxide monoradical. ${ }^{[46]}$ These results indicate that nitronyl nitroxides are exchange-coupled with a coupling constant significantly greater than the ${ }^{14} \mathrm{~N}$-hyperfine coupling, $\mid \mathrm{J} /$ $g \mu_{\mathrm{B}}|\gg| a_{\mathrm{N}} \mid$, i.e., $|J / k| \gg 2 \mathrm{mK}$.

At $140 \mathrm{~K}$, the EPR spectrum of $\mathbf{1}$ in toluene/chloroform glass shows a single peak in the $\left|\Delta m_{\mathrm{s}}\right|=1$ region, with a peak-to-
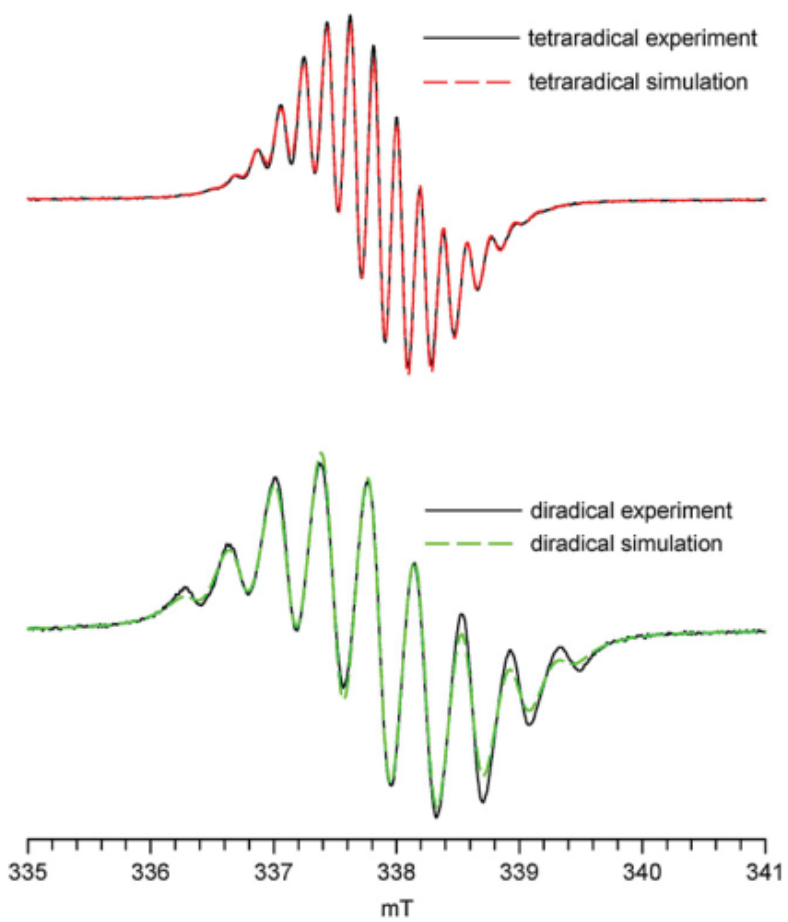

Figure 6. EPR (X-Band) spectra of $0.55 \mathrm{mM}$ tetraradical 1 (top plot) and $0.65 \mathrm{mM}$ diradical 2 (bottom plot) in toluene/chloroform (4:1) at $296 \mathrm{~K}$. Numerical fits (red dashed line) to the experimental spectra correspond to single tetraradical (top plot) and diradical (bottom plot) species; correlation coefficients are 0.999 and 0.995 , respectively. The values for the variable parameters for tetraradical are: Lorentzian linewidth of $0.117 \mathrm{mT}, g$-shift of $-0.145 \mathrm{mT}(g$-value $=2.0068),{ }^{14} \mathrm{~N}$-splitting of $0.188 \mathrm{mT}$ for eight nuclei. The values for the variable parameters for diradical are: Lorentzian linewidth of $0.216 \mathrm{mT}, g$-shift of $-0.147 \mathrm{mT}(g$-value $=2.0065),{ }^{14} \mathrm{~N}$-splitting of $0.376 \mathrm{mT}$ for four nuclei. peak linewidth of about $0.14 \mathrm{mT}$; the spectral envelope of the $\left|\Delta m_{\mathrm{s}}\right|=1$ region is only about doubled in size, compared to the solution phase spectrum for the same sample (Figure 7). Furthermore, the $\left|\Delta m_{\mathrm{s}}\right|=2$ transition could not be detected. These results suggest that tetraradical 1 possesses a rather small zero-field splitting $(|D / h c|)$ that is compatible with a relatively high average symmetry for the disposition of nitronyl nitroxide radicals. Similar EPR spectra, consisting of a single peak in the $\left|\Delta m_{\mathrm{s}}\right|=1$ region with a peak-to-peak linewidth of about $0.20 \mathrm{mT}$, were reported for the corresponding nitroxide tetraradical. ${ }^{[24] \&[25]}$

The EPR spectrum of 2 in toluene/chloroform glass at $140 \mathrm{~K}$ possesses four symmetrically disposed broad peaks in the $\left|\Delta m_{\mathrm{s}}\right|=1$ region and a barely detectable $\left|\Delta m_{\mathrm{s}}\right|=2$ transition. The spectral simulation of the $\left|\Delta m_{\mathrm{s}}\right|=1$ region gives $|D / h c|=$ $4.1 \times 10^{-3} \mathrm{~cm}^{-1}$ and $|E / h c|=0 \mathrm{~cm}^{-1}$ as zero-field splitting parameters (Figure 7). The value of $|D / h c|=4.1 \times 10^{-3} \mathrm{~cm}^{-1}$ is about three times smaller than $|D / h c|=1.39 \times 10^{-2} \mathrm{~cm}^{-1}$ in the corresponding nitroxide diradical. ${ }^{[24]}$ The decrease of $|D / h c|$ may be ascribed to the increased radical-radical distance and greater delocalization of spin density over the ONCNO moiety in 2 . Based upon the point-dipole approximation, ${ }^{[47]}$ the increase of radical-radical distance from 5.5 to $7.5 \AA$, as estimated by X-ray crystallography, should contribute to the decrease of $|D / h c|$ by a factor of 2.5 .
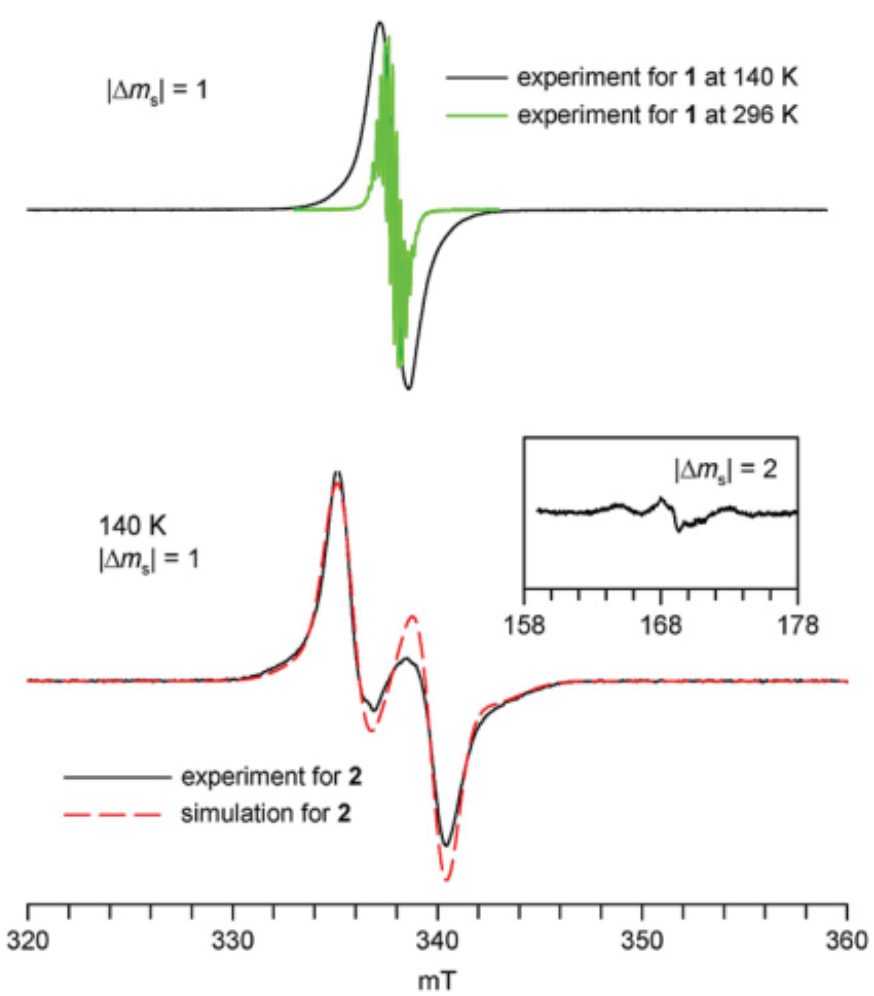

Figure 7. EPR (X-Band) spectra of nitronyl nitroxide tetraradical $1(0.55 \mathrm{mM}$, $9.4893 \mathrm{GHz}$, top plot) and diradical $2(0.65 \mathrm{mM}, 9.4898 \mathrm{GHz}$, bottom plot $)$ in toluene/chloroform $(4: 1)$ at $140 \mathrm{~K}$. For 2 , the spectral simulation of the $\left|\Delta m_{\mathrm{s}}\right|$ $=1$ region is shown as red trace. The fitting parameters for the spectral simulation to the $S=1$ state are: $|D / h c|=4.1 \times 10^{-3} \mathrm{~cm}^{-1},|E / h c|=0 \mathrm{~cm}^{-1}, g_{x}=$ 2.0073, $g_{y}=2.0073, g_{z}=2.0035$, Gaussian line $\left(L_{X}=1.3 \mathrm{mT}, L_{y}=1.7 \mathrm{mT}, L_{z}\right.$ $=3.0 \mathrm{mT}$ ). 
The two outermost peaks are especially broad in $\mathbf{2}$, as estimated by the Gaussian linewidth of $0.30 \mathrm{mT}$ in the spectral simulation (Figure 7). Analogously to the corresponding nitroxide diradical, this broadness may be associated with the unresolved ${ }^{14} \mathrm{~N}$-hyperfine coupling, suggesting that the nitronyl nitroxide moieties in $\mathbf{2}$ may be adopting approximately cofacial conformation in toluene/chloroform glass. ${ }^{[24]}$

The EPR spectra support structural assignments for $\mathbf{1}$ and $\mathbf{2}$, and provide the lower bound estimate for $|J / k| \gg 2 \mathrm{mK}$, similar to that in the corresponding nitroxide tetraradical and diradical. An approximate upper bound for $|J|$ may be determined by magnetic studies of $\mathbf{1}$ and $\mathbf{2}$.

\subsection{Magnetic studies of 1 and 2 in solution and in the solid state}

Magnetization $(M)$ is measured as a function of magnetic field ( $H=0-5 \times 10^{4} \mathrm{Oe}$ and $T=1.8,3$, and $\left.5 \mathrm{~K}\right)$ and temperature $(T$ $=1.8-300 \mathrm{~K}$ at $H=30,000,5000$, or $500 \mathrm{Oe}$ ). The $M$ versus $H$ and $M$ versus $T$ data are plotted as the $M / M_{\text {sat }}$ versus $H / T$ and the $\chi T$ versus $T$, respectively, where $M_{\text {sat }}$ is the magnetization at saturation and $\chi$ is the paramagnetic susceptibility.

For tetraradical 1 and diradical $\mathbf{2}$ in chloroform, the value of $\chi T$ is constant in the $30-150 \mathrm{~K}$ range, with values of $\chi T=1.49$
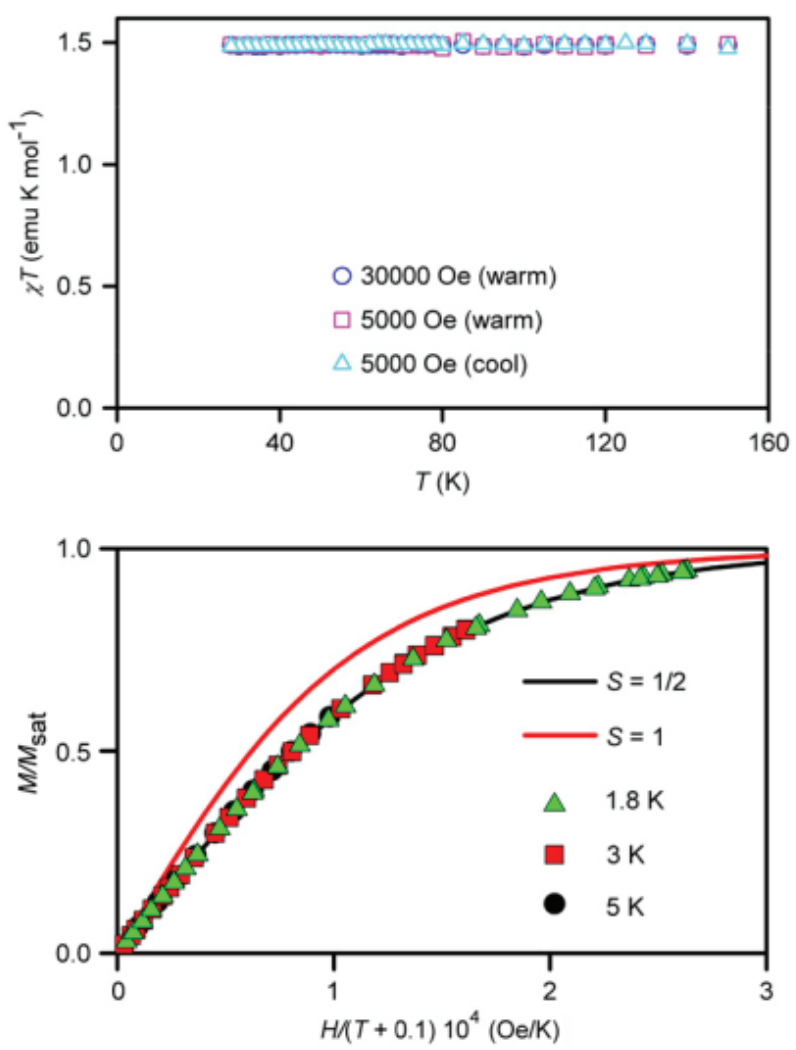

Figure 8. SQUID magnetometry for $5 \mathrm{mM}$ tetraradical 1 in $\mathrm{CHCl}_{3}$. Top plot corresponds to $\chi T$ versus $T$ in both cooling and warming modes, with $\chi T=$ $1.49 \mathrm{emu} \mathrm{K} \mathrm{mol}{ }^{-1}$ in the high temperature range. Bottom plot corresponds to $M / M_{\text {sat }}$ versus $H /(T-\theta)$, with solid lines showing plots of Brillouin functions with $S=1 / 2$ and $S=1$; numerical fits to the Brillouin functions with $\theta=$ $-0.1 \mathrm{~K}$ give $S=0.50$ and $M_{\mathrm{sat}}=0.98 \mu_{\mathrm{B}}$ at $1.8,3$, and $5 \mathrm{~K}$. and $0.72-0.73 \mathrm{emu} \mathrm{K} \mathrm{mol}{ }^{-1}$ for $\mathbf{1}$ and 2, respectively (Figure 8 and Figure S6, Supplementary data). Similarly, the ${ }^{1} \mathrm{H}$ NMR-based Evans method gives quantitative values of $\chi T$ $\approx 1.3-1.5$ and $0.8 \mathrm{emu} \mathrm{K} \mathrm{mol}{ }^{-1}$ for $\mathbf{1}$ and $\mathbf{2}$ in chloroform at room temperature (295-300 K), respectively. Within accuracy of the Evans method, ${ }^{[43], ~[43 a] ~ \& ~[43 b] ~ t h e s e ~ v a l u e s ~ a r e ~ i n ~ g o o d ~}$ agreement with the values of $\chi T$ at $T>30 \mathrm{~K}$ for solids $\mathbf{1}$ and $\mathbf{2}$ (Figure 9 and Figure 10). These values of $\chi T$ at high temperatures, $T>30 \mathrm{~K}$, are in excellent agreement with the theoretical, spin-only $\chi T=1.50$ and $0.75 \mathrm{emu} \mathrm{K} \mathrm{mol}{ }^{-1}$ for tetraradicals and diradicals, with independent $S=1 / 2$ radicals.

At low temperatures, the $\chi T$ versus $T$ plots for $\mathbf{1}$ and $\mathbf{2}$ in the solid state show downward turns, consistent with antiferromagnetic exchange coupling, corresponding to a mean-field parameters, $\theta \approx-0.5$ and $-0.3 \mathrm{~K}$, respectively. In order to distinguish whether these exchange couplings are intramolecular or intermolecular, magnetic data in the solid state and in dilute solutions at low temperature ranges should be compared. ${ }^{[24]}$ However, the studies of $\mathbf{1}$ and $\mathbf{2}$ in solution are constrained by their low solubility in common organic solvents and magnetic data in solution could only be obtained in chloroform. The $\chi T$ versus $T$ plots for $T<30 \mathrm{~K}$ could not be obtained because of the apparent time-dependent magnetic behavior of the radicals in a chloroform matrix. Therefore, low temperature magnetic
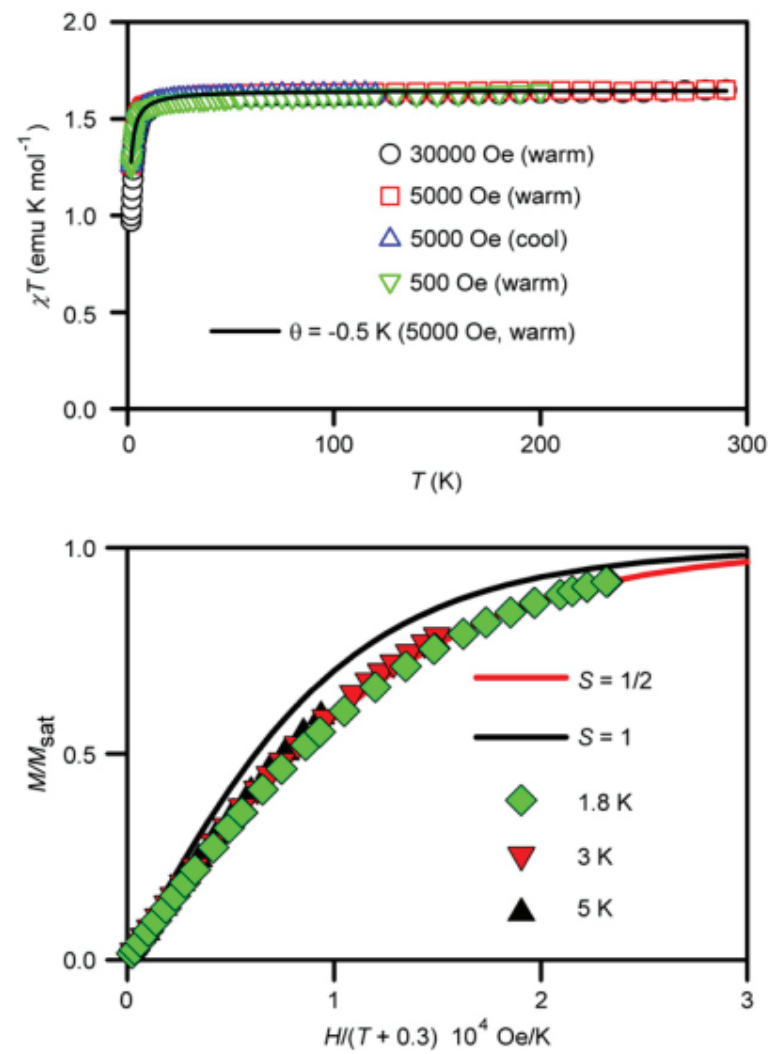

Figure 9. SQUID magnetometry for polycrystalline tetraradical 1. Top plot corresponds to $\chi T$ versus $T$ in both cooling and warming modes; the solid line shows a numerical fit to the $S=1 / 2$ Brillouin function with $\theta=-0.5 \mathrm{~K}$. Bottom plot corresponds to $M / M_{\text {sat }}$ versus $H /(T-\theta)$, with solid lines showing plots of Brillouin functions with $S=1 / 2$ and $S=1$; numerical fits to the Brillouin functions with $\theta=-0.3 \mathrm{~K}$ give $S \approx 0.5$ and $M_{\text {sat }} \approx 1.0 \mu_{\mathrm{B}}$ at 1.8 and $3 \mathrm{~K}$. 
data can only be obtained at constant temperature, after long annealing of the matrix at each temperature. In this way, the $M / M_{\text {sat }}$ versus $H /(T-\theta)$ plots at $T=1.8,3$, and $5 \mathrm{~K}$ were obtained for $5 \mathrm{mM} 1$ in chloroform and $20 \mathrm{mM} 2$ in chloroform (Figure 8 and Figure S6); for both 1 and $2, \theta \approx-0.1 \mathrm{~K}$, with $S$ $\approx 0.5$ and $M_{\text {sat }}=0.9-1.0 \mu_{\mathrm{B}}$, is obtained. ${ }^{[48]}$ These values of $\theta$ may be compared to $\theta \approx-0.3 \mathrm{~K}$, as well as $S \approx 0.5$ and $M_{\text {sat }} \approx$ $1.0 \mu_{\mathrm{B}}$, obtained from analogous plots for $\mathbf{1}$ and $\mathbf{2}$ in the solid state (Figure 9 and Figure 10).

These results indicate that antiferromagnetic exchange coupling between the radicals in $\mathbf{1}$ and $\mathbf{2}$ is very small and most likely intermolecular. In chloroform solution, the magnetic data for both 1 and $\mathbf{2}$ resemble paramagnetic behavior of four and two independent spins $S=1 / 2$, respectively, even at the lowest measurement temperature of $1.8 \mathrm{~K}$. This provides the upper limit of $|J / k| \ll 0.5 \mathrm{~K}$, most likely $|J / k|<0.2 \mathrm{~K}$, for the strength of intramolecular exchange coupling between the radicals.

\subsection{Crystal packing of 1}

The crystal packing of tetraradical $\mathbf{1}$ may be described in terms of alternating layers of calix[4]arene macrocycles and benzene molecules in the approximate $a c$ - or (010)-planes (Figure 11, Figures S1-S3, Supplementary data).
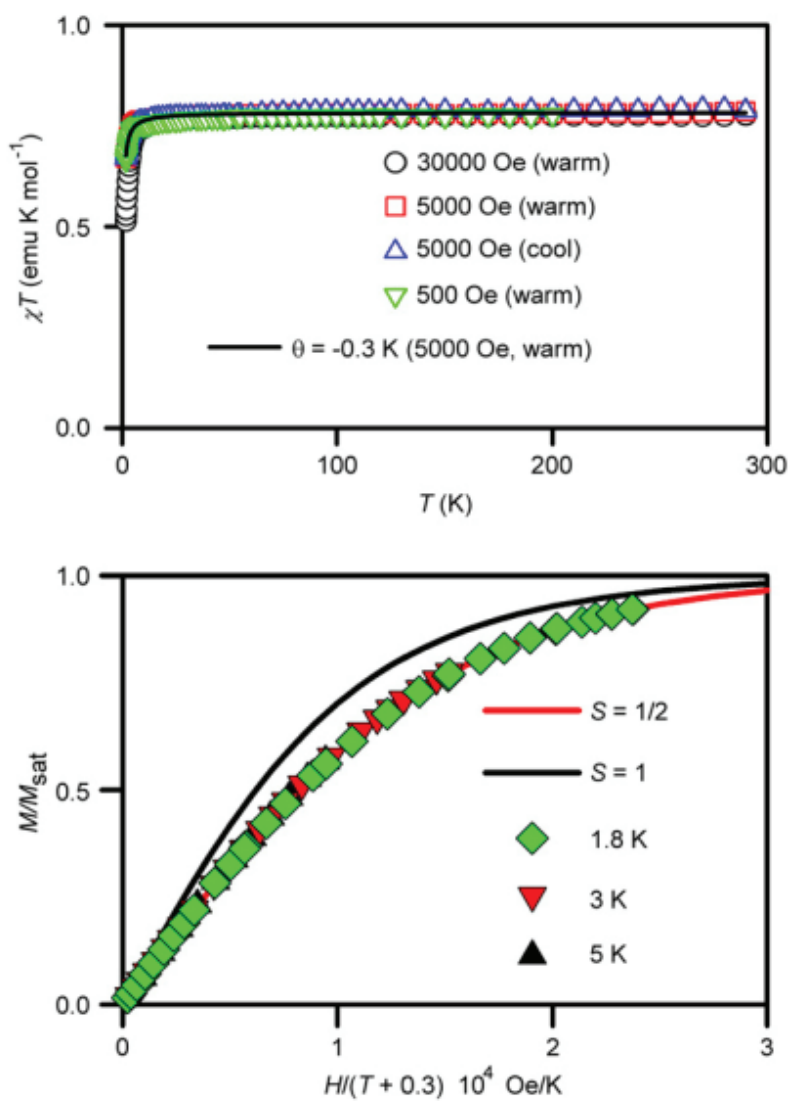

Figure 10. SQUID magnetometry for polycrystalline diradical 2. Top plot corresponds to $\chi T$ versus $T$ in both cooling and warming modes; the solid line shows a numerical fit to the $S=1 / 2$ Brillouin function with $\theta=-0.3 \mathrm{~K}$. Bottom plot corresponds to $M / M_{\text {sat }}$ versus $H /(T-\theta)$, with solid lines showing plots of Brillouin functions with $S=1 / 2$ and $S=1$; numerical fits to the Brillouin functions with $\theta=-0.3 \mathrm{~K}$ give $S \approx 0.50$ and $M_{\text {sat }} \approx 1.0 \mu_{\mathrm{B}}$ at 1.8 and $3 \mathrm{~K}$.
For 1, multiple intermolecular radical-radical $\mathrm{O} \cdots \mathrm{O}$ distances in the 3.6-4.1 $\AA$ range may be identified within each layer of macrocycles. One pair of nitronyl nitroxides has relatively short radical-radical $\mathrm{O} \cdots \mathrm{O}$ distance, O2A-O6B $=3.607 \AA$. Another pair of nitronyl nitroxides possesses radical-radical $\mathrm{O} \cdots \mathrm{O}$ distances of $\mathrm{O} 10 \mathrm{~A}-\mathrm{O} 1 \mathrm{~B}=3.636 \AA$ and $\mathrm{O} 10 \mathrm{~A}-\mathrm{O} 2 \mathrm{~B}$ $=4.088 \AA$. For both pairs, significant antiferromagnetic exchange coupling is expected, and in the second pair, exchange coupling may be amplified by the presence of two parallel exchange coupling pathways. ${ }^{[49]}$ Intermolecular antiferromagnetic exchange coupling may contribute to the more negative values of the mean-field parameter $\theta$ for the magnetic data of $\mathbf{1}$ in the solid state.

\section{Radical- $m$-phenylene-C( $\left(\mathrm{sp}^{3}\right)-m$-phenylene-radical ex- change coupling pathway}

In 2000, Frank and co-workers reported the synthesis and characterization of a nitronyl nitroxide-based spiroconjugated diradical, shown in Figure 12. ${ }^{[50]}$ The magnetic studies of the diradical in the solid state indicated a relatively large antiferromagnetic exchange coupling, which was assigned to the through-bond exchange coupling with the coupling constant $J / k=-3 \mathrm{~K}$ (singlet-triplet gap, $2|J / k|=6 \mathrm{~K}$ ). ${ }^{[50]}$ In addition, plots of the EPR signal intensity versus $1 / T$ for the diradical in dilute organic matrices showed curvatures at low temperatures that were consistent with $J / k \approx-3 \mathrm{~K} .{ }^{[50]}$ These values

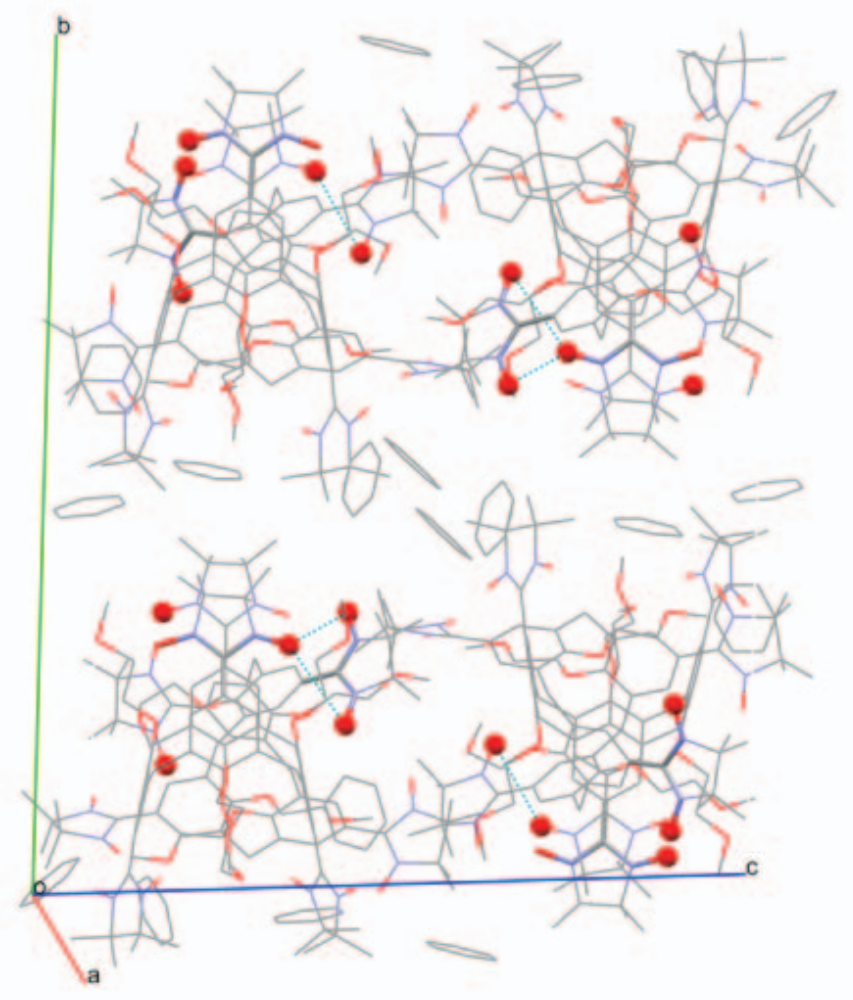

Figure 11. Crystal packing of nitroxide tetraradical 1. Disorder and hydrogen atoms are omitted for clarity. Oxygen atoms with short radical-radical $\mathrm{O} \cdots \mathrm{O}$ distances, 3.607-4.088 $\AA$, are depicted as ball-and-stick model. Pairs of the ONCNO moieties connected with two short $\mathrm{O} \cdots \mathrm{O}$ distances are depicted as stick model. 


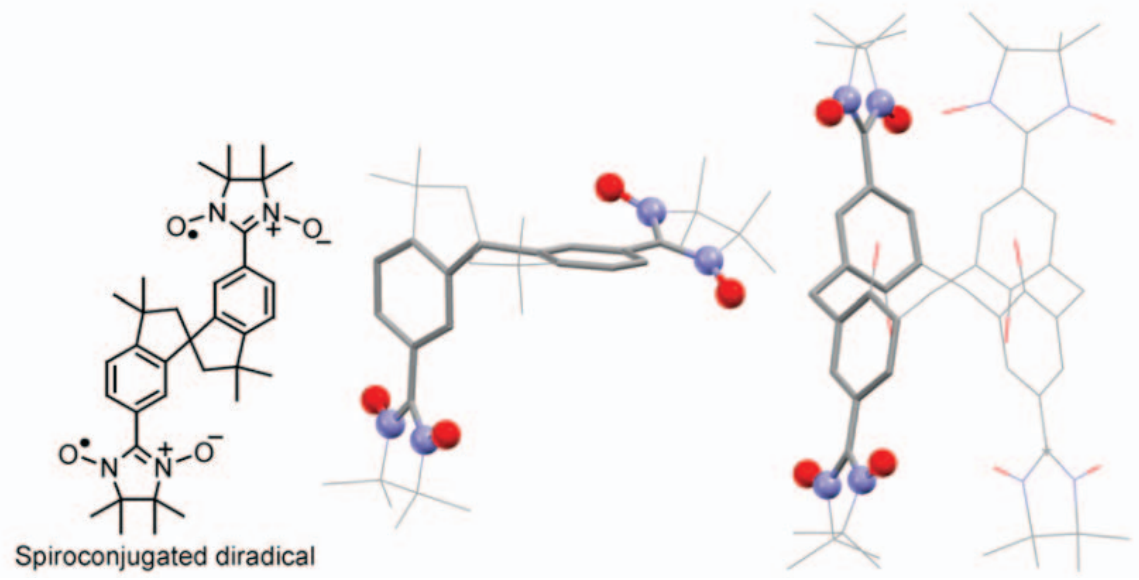

Figure 12. The nitronyl nitroxide- $m$-phenylene- $\mathrm{C}\left(\mathrm{sp}^{3}\right)-m$-phenylene-nitronyl nitroxide exchange coupling pathways (displayed as stick model) in spiroconjugated diradical and in 1,3-alternate calix[4]arene tetraradical 1. The NO moieties are shown as stick-and-ball model. Hydrogen atoms and methoxyethylene chains in tetraradical $\mathbf{1}$ are omitted for clarity.

of $|J / k|$ are larger by at least one order of magnitude than $|J|$ $k \mid \ll 0.5 \mathrm{~K}$, determined for nitronyl nitroxide tetraradical 1 . It should be noted that the through-bond exchange coupling pathway, radical- $m$-phenylene- $\mathrm{C}\left(\mathrm{sp}^{3}\right)-m$-phenylene-radical, in the spiroconjugated diradical and tetraradical $\mathbf{1}$ is identical but the conformations of the exchange coupling pathways are significantly different (Figure 12) ${ }^{24}$ However, it might be possible that the intermolecular exchange coupling in the solid state (in the magnetic studies) and the microwave saturation at low temperatures in the EPR studies significantly affected the

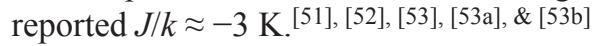

\section{Conclusion}

Exchange coupling in 1,3-alternate calix[4]arene nitronyl nitroxide tetraradical and diradical was studied. In the solid state, weak antiferromagnetic coupling, that is stronger than the one in solution, is found between neighboring molecules. In solution, the exchange coupling constants $(|J / k|)$ are much larger than ${ }^{14} \mathrm{~N}$-hyperfine splitting for nitronyl nitroxides but they are much smaller than those determined for the corresponding nitroxide radicals, i.e., $0.5 \mathrm{~K} \gg|J / k| \gg 2 \mathrm{mK}$. Much weaker intramolecular exchange coupling in the nitronyl nitroxide tetraradical and diradical, compared to that in the corresponding nitroxide tetraradical and diradical, correlates to their distinct structures and spin density distributions. 1,3-Alternate calix[4]arene nitronyl nitroxide tetraradical and diradical provide a model system for the study of electron spin relaxation in the presence of weak radical-radical interactions.

\section{Experimental section}

\subsection{Materials and special procedures}

Ether, tetrahydrofuran (THF), and 2-methyltetrahydrofuran (2MeTHF) were freshly distilled from sodium/benzophenone prior to use. $n$-BuLi in hexane and $t$-BuLi in pentane were obtained from either Aldrich or Acros; concentration of the organolithiums was determined by titration with $N$-pivaloyl-o-toluidine. ${ }^{[54]}$ Perdeuterated solvents for NMR spectroscopy were obtained from
Cambridge Isotope Laboratories. All other commercially available chemicals were obtained from Aldrich or Acros. Prior to use, benzaldehyde and $p$-anisaldehyde were subjected to the general method for purification of aldehydes. ${ }^{[55]} 5,11,17,23$-Tetrabromo-25,26,27,28-tetrakis(methoxyethoxy)calix[4]arene (3) in the locked 1,3-alternate conformation ${ }^{[37]}$ was prepared using previously reported procedures. ${ }^{[24]}$ 2,3-Bis(hydroxyamino)-2,3-dimethylbutane was obtained by reduction of the corresponding dinitro compound using $\mathrm{Al} / \mathrm{Hg} .{ }^{[39]}$ Column chromatography was carried out with flash silica gel, particle size $40-63 \mu \mathrm{m}$, obtained from EMD chemicals or with TLC grade silica gel (Aldrich) using 0-20 psig pressure. Preparative TLC (PTLC) was carried out using Analtech silica plates (tapered with a preadsorbent zone). Standard Schlenk techniques for synthesis under inert atmosphere were employed.

\subsection{NMR spectroscopy and other analyses}

NMR spectra were obtained in $\mathrm{CDCl}_{3}$ or DMSO- $d_{6}$ using Bruker spectrometers $\left({ }^{1} \mathrm{H}, 400\right.$ and $\left.600 \mathrm{MHz}\right)$. The chemical shift references were as follows: $\left({ }^{1} \mathrm{H}\right) \mathrm{CHCl}_{3}, 7.26 \mathrm{ppm}$; $\left.{ }^{13} \mathrm{C}\right) \mathrm{CDCl}_{3}$, $77.0 \mathrm{ppm}\left(\mathrm{CDCl}_{3}\right)$; $\left({ }^{1} \mathrm{H}\right)$ DMSO- $d_{5}, 2.50 \mathrm{ppm}$; $\left({ }^{13} \mathrm{C}\right)$ DMSO- $d_{5}$, $38.50 \mathrm{ppm}$ (DMSO- $d_{6}$ ). Typical 1D FID was subjected to exponential multiplication with an exponent of $0.1 \mathrm{~Hz}\left(\right.$ for $\left.{ }^{1} \mathrm{H}\right)$ and 1.0 $2.0 \mathrm{~Hz}$ (for ${ }^{13} \mathrm{C}$ ). Values of the magnetic moment and $\chi T$ were obtained in chloroform using the ${ }^{1} \mathrm{H}$ NMR-based Evans method. ${ }^{[43]}$, [43a], \& [43b] Concentric NMR tubes were used (Wilmad, cat. No. WGS-5BL). The outer NMR tube contained a solution of the paramagnetic sample in an approximately $1: 1(\mathrm{v} / \mathrm{v})$ mixture of $\mathrm{CDCl}_{3}$ and $\mathrm{CHCl}_{3}$ with accurately determined concentration and the inner concentric tube contained pure $\mathrm{CHCl}_{3}$. Diamagnetic susceptibility of $\mathrm{CHCl}_{3}\left(\chi_{\text {dia }}=-0.497 \times 10^{-6} \mathrm{emu} \mathrm{g}^{-1}\right)$ was used. IR spectra were obtained using a Nicolet Avatar 360 FTIR instrument, equipped with an ATR sampling accessory (Spectra Tech, Inc.). A few drops of the compound in $\mathrm{CH}_{2} \mathrm{Cl}_{2}$ were applied to the surface of a ZnSe ATR plate horizontal parallelogram ( $45^{\circ}$, Wilmad). After the solvent evaporated, the spectrum was acquired (128 scans, $4 \mathrm{~cm}^{-1}$ resolution). FABMS analyses were carried out at the Nebraska Center for Mass Spectrometry, using 3-nitrobenzyl alcohol (3-NBA) as a matrix. 


\subsection{X-ray crystallography}

Crystals of 1 and $\mathbf{2}$ were obtained by slow evaporation of benzene/heptane.

The data were collected on a Bruker SMART6000 system at the Indiana University and at beamline 15ID, ChemMatCARS, at the Advanced Photon Source, Argonne National Laboratory in Chicago. For the data collection, single crystals were placed onto the tip of a $0.1 \mathrm{~mm}$ diameter glass capillary.

Additional description of the X-ray crystallographic data may be found in Refs. [40], [41. (a), (b) and (c)], as well as in the Supplementary data.

\subsection{EPR spectroscopy}

CW X-band EPR spectra for $\mathbf{1}$ and $\mathbf{2}$ in solution were acquired on Bruker EMX instrument, equipped with a frequency counter and nitrogen flow temperature control (130-300 K). The samples were contained in 4-mm O.D. EPR quartz tubes, equipped with high-vacuum PTFE stopcocks. Solvent was vacuum transferred into the tube, to form a homogeneous solution after attaining ambient temperature. Spectra were obtained using dual mode cavity, with the oscillating magnetic field perpendicular $\left(\mathrm{TE}_{102}\right)$ to the swept magnetic field. The $g$-values were referenced using DPPH ( $g=2.0037$, powder, Aldrich).

\subsection{SQUID magnetometry}

Quantum Design (San Diego, CA) MPMS5S (with continuous temperature control) was used.

For solution samples, tetraradical 1 or diradical $\mathbf{2}$ was loaded into homemade 5-mm O.D. EPR quality quartz tubes, equipped with high-vacuum PTFE stopcocks. The tubes were modified to possess a thin bottom, which is $6 \mathrm{~cm}$ from the end of the tube. ${ }^{[18]}$ \& [24] The tube was placed under vacuum, and then solvent was vacuum transferred. After the tube was flame sealed under vacuum, the frozen samples were rapidly inserted from liquid nitrogen to the magnetometer, with the sample chamber at $10 \mathrm{~K}$, using the well-established procedure for polyarylmethyl polyradicals. ${ }^{[18]}$ Subsequently, a sequence of measurements below the softening point of the matrix $(1.8-150 \mathrm{~K})$ were carried out. Correction for diamagnetism was implemented by extrapolation of the $\chi$ versus $1 / T$ plots, typically, from the $70-140 \mathrm{~K}$ temperature range $\left(R^{2}=0.9999\right)$. In most cases, the warming mode data, with 60-s delays, after a 'stable temperature' was indicated by the MPMS at each temperature, were used for such extrapolations.

For solid state samples, polycrystalline tetraradical 1 or diradical 2 (18-21 mg) was loaded to the 3-piece gelatin capsule. Following the measurements, the capsule was partially emptied (7-8 mg of radical left), and then identical sequences of measurements were carried out for the point-by-point correction for diamagnetism. Additional correction was based upon Pascal constants: $\chi_{\text {dia }}$ $=7.84 \times 10^{-4} \mathrm{emu} \mathrm{mol}^{-1}$ (for 1) and $\chi_{\text {dia }}=6.59 \times 10^{-4} \mathrm{emu} \mathrm{mol}^{-1}$ (for 2). ${ }^{[56]}$

\subsection{EPR spectral simulations and numerical curve fitting for SQUID magnetic data}

The WINEPR SimFonia program (Version 1.25, Bruker) was used for spectral simulations of nitronyl nitroxide diradical 2 in rigid matrices. WinSIM program (Public EPR Software Tools, D. A. O’Brien, D. R. Duling, Y. C. Fann) was used for numerical fitting of solution phase EPR spectra. The SigmaPlot for Windows software package was used for numerical curve fitting of the magnetic data. The reliability of a fit is measured by the parameter dependence, which is defined as follows: dependence $=1-($ variance of the parameter, other parameters constant)/(variance of the parameter, other parameters changing)). Values close to 1 indicate an overparametrized fit.

\subsection{Tetraaldehyde $4: 5,11,17,23$-tetraformyl-25,26,27,28-tetra kis(methoxyethoxy)calix[4] $\operatorname{arene}^{[38]}$}

$t$-BuLi in pentane (titrated, $1.58 \mathrm{M}, 1.57 \mathrm{~mL}, 2.5 \mathrm{mmol}$ ) was added to a solution of tetrabromocalix[4]arene $3(0.301 \mathrm{~g}$, $0.310 \mathrm{mmol})$ in THF $(20 \mathrm{~mL})$ at $-78{ }^{\circ} \mathrm{C}$. The resultant bright orange solution was stirred at $-78^{\circ} \mathrm{C}$ for $2 \mathrm{~h}$, and then warmed up to $-20{ }^{\circ} \mathrm{C}$ for $15 \mathrm{~min}$. The reaction was re-cooled to $-78^{\circ} \mathrm{C}$, and neat $N$-methylformanilide $(0.168 \mathrm{~g}, 1.24 \mathrm{mmol})$ was added. The reaction mixture was left to warm up to room temperature overnight. It was then taken up in excess chloroform $(\sim 100 \mathrm{~mL})$, and the organic layer was washed with water $(3 \times 50 \mathrm{~mL})$ and dried with $\mathrm{MgSO}_{4}$. Evaporation of the solvent and flash chromatography (silica, 20-25\% ethyl acetate in benzene) gave tetraaldehyde 4 (189.5 mg, 79\%). From two other reactions carried out using 100.0 and $401.7 \mathrm{mg}$ of the tetrabromocalix[4]arene 3, tetraaldehyde 4 was obtained in $51 \%(40.3 \mathrm{mg})$ and $70 \%$ $(0.222 \mathrm{~g})$ yields, respectively; for these two reactions, purification was based upon triturating the crude with ether. Mp 172$174{ }^{\circ} \mathrm{C}$ (under $\mathrm{N}_{2}$ ). ${ }^{1} \mathrm{H}$ NMR $\left(400 \mathrm{MHz}, \mathrm{CDCl}_{3}\right.$ ): $\delta 9.673$ (s, $4 \mathrm{H}, \mathrm{CHO}), 7.657$ (s, 8H, $\mathrm{ArH}), 3.997\left(\mathrm{~m}, 8 \mathrm{H}, \mathrm{OCH}_{2}\right), 3.871(\mathrm{~m}$, $\left.8 \mathrm{H}, \mathrm{OCH}_{2}\right), 3.633\left(\mathrm{~s}, 8 \mathrm{H}, \mathrm{ArCH}_{2}\right), 3.621\left(\mathrm{~s}, 12 \mathrm{H}, \mathrm{OCH}_{3}\right) .{ }^{13} \mathrm{C}$ NMR $\left(100 \mathrm{MHz}, \mathrm{CDCl}_{3}\right): \delta 191.8,160.7,133.9,132.0,131.0$, 72.7, 71.9, 59.0, 33.3. IR (ZnSe, $\left.\mathrm{cm}^{-1}\right)$ : 2980, 2933, 2878, 1684, $1587,1452,1432,1308,1274,1222,1120,1052,1027$. The ${ }^{1} \mathrm{H}$ and ${ }^{13} \mathrm{C}$ NMR spectra were coinciding with those previously reported for tetraaldehyde 4. $^{[38]}$

\subsection{Dialdehyde 5: 5,17-diformyl-11,23-dibromo-25,26,27,28-t etrakis(methoxyethoxy)calix[4]arene}

$n$-BuLi in hexane (titrated, $2.45 \mathrm{M}, 0.52 \mathrm{~mL}, 1.30 \mathrm{mmol}$ ) was added to a solution of tetrabromocalix[4]arene $3(0.614 \mathrm{~g}$, $0.630 \mathrm{mmol})$ in THF $(37 \mathrm{~mL})$ at $-78^{\circ} \mathrm{C}$. The resultant orange solution was stirred at $-78^{\circ} \mathrm{C}$ for $2 \mathrm{~h}$, and then warmed to $-20^{\circ} \mathrm{C}$ for $15 \mathrm{~min}$. The color of the solution changed to a dark red. The reaction mixture was then cooled to $-78^{\circ} \mathrm{C}$, and then neat $N$-methylformanilide $(0.204 \mathrm{~g}, 1.51 \mathrm{mmol})$ was added, to give a colorless solution. The reaction mixture was left to warm up to room temperature overnight. After removal of solvents under reduced pressure, a yellow residue was obtained, and then dissolved in dichloromethane; the organic layer was then washed with distilled water three times. The or- 
ganic layer was dried over $\mathrm{MgSO}_{4}$ and concentrated in vacuo to give yellowish-white crude $(0.795 \mathrm{~g})$. This crude was combined with the crude $(1.108 \mathrm{~g})$ from another reaction carried out on $1.0 \mathrm{~g}$ of the tetrabromocalix[4]arene 3 , and then purified by flash chromatography (silica, $5-10 \%$ ether in chloroform) to yield dialdehyde 5 (0.699 g, 48\%). Column chromatography of the combined crudes from two other reactions, done on 50.0 and $0.500 \mathrm{~g}$ of the tetrabromocalix[4]arene 3 , followed by treatment with ether, gave dialdehyde $5(0.181 \mathrm{~g}, 37 \%)$. Mp 217-220 ${ }^{\circ} \mathrm{C}$ (under $\mathrm{N}_{2}$ ). ${ }^{1} \mathrm{H}$ NMR $\left(400 \mathrm{MHz}, \mathrm{CDCl}_{3}\right.$ ): $\delta$ 9.683 (s, 2H, CHO), 7.662 (s, 4H, ArH), 7.323 (s, 4H, ArH), $3.927\left(\mathrm{~m}, 8 \mathrm{H}, \mathrm{OCH}_{2}\right), 3.83-3.79\left(\mathrm{~m}, 8 \mathrm{H}, \mathrm{OCH}_{2}\right), 3.622(\mathrm{~s}, 3 \mathrm{H}$, $\left.\mathrm{OCH}_{3}\right), 3.594\left(\mathrm{~s}, 3 \mathrm{H}, \mathrm{OCH}_{3}\right), 3.595,3.502(\mathrm{AB}, J=13 \mathrm{~Hz}, 8 \mathrm{H}$, $\left.\mathrm{ArCH}_{2}\right) \cdot{ }^{13} \mathrm{C}$ NMR $\left(100 \mathrm{MHz}, \mathrm{CDCl}_{3}\right): \delta 191.8,160.7,154.4$, 134.6, 133.7, 133.0, 132.5, 130.7, 115.2, 72.4, 72.3, 71.9, 71.9, 59.3, 59.0, 33.7. HR-FABMS (3-NBA matrix) $\mathrm{m} / z$ (ion type, $\%$ RA for $m / z=868-875$, deviation for the formula): 874.1553 $\left([\mathrm{M}+2 \mathrm{H}+4]^{+}, 22 \%, 2.4\right.$ ppm for $\left.{ }^{12} \mathrm{C}_{42}{ }^{1} \mathrm{H}_{48}{ }^{16} \mathrm{O}_{10}{ }^{81} \mathrm{Br}_{2}\right), 874.1553$ $\left([\mathrm{M}+\mathrm{H}+5]^{+}, \quad 22 \%, \quad-2.8 \mathrm{ppm}\right.$ for $\left.{ }^{12} \mathrm{C}_{41}{ }^{13} \mathrm{C}_{1}{ }^{1} \mathrm{H}_{47}{ }^{16} \mathrm{O}_{10}{ }^{81} \mathrm{Br}_{2}\right)$, $873.1507\left([\mathrm{M}+\mathrm{H}+4]^{+}, 58 \%,-1.4 \mathrm{ppm}\right.$ for $\left.{ }^{12} \mathrm{C}_{42}{ }^{1} \mathrm{H}_{47}{ }^{16} \mathrm{O}_{10}{ }^{81} \mathrm{Br}_{2}\right)$, $872.1516\left([\mathrm{M}+\mathrm{H}+3]^{+}, 57 \%, 3.7 \mathrm{ppm}\right.$ for ${ }^{12} \mathrm{C}_{41}{ }^{12} \mathrm{C}_{1}{ }^{17} \mathrm{H}_{47}{ }^{16} \mathrm{O}_{10}{ }^{79} \mathrm{Br}$ $\left.{ }_{1}^{81} \mathrm{Br}_{1}\right), 871.1508([\mathrm{M}+\mathrm{H}+2]]^{+}, 100 \%, 0.9 \mathrm{ppm}$ for ${ }^{12} \mathrm{C}_{42}{ }^{1} \mathrm{H}_{47}{ }^{16} \mathrm{O}$ $\left.{ }_{10}^{79} \mathrm{Br}_{1}{ }^{81} \mathrm{Br}_{1}\right), 871.1508\left([\mathrm{M}+3]^{+}, 100 \%,-4.3 \mathrm{ppm}\right.$ for ${ }^{12} \mathrm{C}_{41}{ }^{13} \mathrm{C}$ $\left.{ }_{1}^{1} \mathrm{H}_{46}{ }^{16} \mathrm{O}_{10}{ }^{79} \mathrm{Br}_{1}{ }^{81} \mathrm{Br}_{1}\right), 870.1483\left([\mathrm{M}+2]^{+}, 57 \%,-5.2 \mathrm{ppm}\right.$ for ${ }^{12}$ $\left.\mathrm{C}_{42}{ }^{1} \mathrm{H}_{46}{ }^{16} \mathrm{O}_{10}{ }^{79} \mathrm{Br}_{1}{ }^{81} \mathrm{Br}_{1}\right), 869.1514\left([\mathrm{M}+\mathrm{H}]^{+}, 54 \%, 2.5 \mathrm{ppm}\right.$ for $\left.{ }^{12} \mathrm{C}_{42}{ }^{16} \mathrm{H}_{47}{ }^{16} \mathrm{O}_{10}{ }^{79} \mathrm{Br}_{2}\right), 869.1514\left([\mathrm{M}+1]^{+}, 54 \%,-2.6\right.$ ppm for ${ }^{1}$ $\left.{ }^{2} \mathrm{C}_{41}{ }^{13} \mathrm{C}_{1}{ }^{1} \mathrm{H}_{46}{ }^{16} \mathrm{O}_{10}{ }^{79} \mathrm{Br}_{2}\right), 868.1445\left([\mathrm{M}]^{+}, 17 \%, 1.4 \mathrm{ppm}\right.$ for $\left.{ }^{12} \mathrm{C}_{42}{ }^{1} \mathrm{H}_{46}{ }^{16} \mathrm{O}_{10}{ }^{79} \mathrm{Br}_{2}\right)$. IR $\left(\mathrm{ZnSe}, \mathrm{cm}^{-1}\right): 2979,2923,2886,1689$, 1597, 1588, 1450, 1306, 1200, 1124, 1054, 1029.

\subsection{Tetrakis(1,3-dihydroimidazolidine) $6: 5,11,17,23$-tet- rakis(1,3-dihydroxy-4,4,5,5-tetramethylimidazolin-2-yl)- 25,26,27,28-tetrakis(methoxyethoxy)calix[4]arene}

A mixture of tetraaldehyde $4(0.173 \mathrm{~g}, 0.224 \mathrm{mmol}), 2,3-$ bis(hydroxyamino)-2,3-dimethylbutane $(0.327 \mathrm{~g}, 2.21 \mathrm{mmol})$, $p$ - $\mathrm{TsOH} \cdot \mathrm{H}_{2} \mathrm{O}(8.4 \mathrm{mg}, 0.044 \mathrm{mmol})$ was evacuated in a Schlenk flask for $30 \mathrm{~min}$. Subsequently, methanol $(1.0 \mathrm{~mL})$ was added under $\mathrm{N}_{2}$, and then the Schlenk flask was transferred to an oil bath at $60-65^{\circ} \mathrm{C}$. The progress of the reaction, it took between 2 and 2.5 days to complete, was followed by TLC (silica, ether/ hexanes); the tetra-condensation product had $R_{f} \approx 0$, even in acetone and in acetonitrile. The crude was then washed with water $(2 \times 3 \mathrm{~mL})$, ethanol containing a drop of $\mathrm{Et}_{3} \mathrm{~N}(1 \times 2 \mathrm{~mL})$, and ether $(3 \times 1 \mathrm{~mL})$ to give tetrakis(1,3-dihydroimidazolidine) $\mathbf{6}$ as a white solid $(0.227 \mathrm{~g}, 79 \%)$. From two other reactions done on $51.0 \mathrm{mg}$ of the tetraaldehyde 4 with methanol $(2 \mathrm{~mL})$ and heating at $65-$ $75^{\circ} \mathrm{C}$, and $0.175 \mathrm{~g}$ of the tetraaldehyde 4 with methanol $(2 \mathrm{~mL})$ and heating at $60-65^{\circ} \mathrm{C}, 6$ was obtained in $91 \%(0.078 \mathrm{~g})$ and $85 \%(0.250 \mathrm{~g})$ yields, respectively. $\mathrm{Mp} \sim 270{ }^{\circ} \mathrm{C}$ (under $\mathrm{N}_{2}, \mathrm{dec}$ ). ${ }^{1} \mathrm{H}$ NMR (400 MHz, DMSO- $\left.d_{6}\right): \delta 7.605$ (s, 8H, OH, exch $\left.\mathrm{D}_{2} \mathrm{O}\right)$, 7.196 (s, 8H, $\mathrm{ArH}), 4.497$ (s, 4H, CH), 3.847 (s, 8H, $\mathrm{ArCH}_{2}$ ), $3.355\left(\mathrm{~s}, 8 \mathrm{H}, \mathrm{OCH}_{2}\right.$, under the $\mathrm{H}_{2} \mathrm{O}$ peak from solvent), 3.104 (s, $\left.12 \mathrm{H}, \mathrm{OCH}_{3}\right), 2.940$ (t, $\left.5.2 \mathrm{~Hz}, 8 \mathrm{H}, \mathrm{OCH}_{2}\right), 1.071\left(\mathrm{~s}, 12 \mathrm{H}, \mathrm{CH}_{3}\right)$, $0.974\left(\mathrm{~s}, 12 \mathrm{H}, \mathrm{CH}_{3}\right) .{ }^{13} \mathrm{C}$ NMR $\left(100 \mathrm{MHz}, \mathrm{DMSO}-d_{6}\right): \delta 156.3$, 135.5, 133.1, 129.0, 90.4, 70.9, 69.4, 66.4, 58.7, 38.4, 25.0, 17.6. LR-FABMS (3-NBA matrix) $m / z$ : calculated for $\mathrm{C}_{68} \mathrm{H}_{104} \mathrm{~N}_{8} \mathrm{O}_{16}$ $[\mathrm{M}]^{+}$1289.6; found 1289.6. IR $\left(\mathrm{ZnSe}, \mathrm{cm}^{-1}\right): 3269,2977,2926$, 1453, 1364, 1203, 1118.
5.10. Bis(1,3-dihydroimidazolidine) 7: 5,17-bis(1,3-dihydroxy-4,4,5,5-tetramethylimidazolin-2-yl)-11,23-dibromo25,26,27,28-tetrakis(methoxyethoxy)calix[4]arene

A mixture of dialdehyde 5 (0.100 g, $0.115 \mathrm{mmol}), 2,3-$ bis(hydroxyamino)-2,3-dimethylbutane ( $85.2 \mathrm{mg}, 0.575 \mathrm{mmol}$ ), $p$ - $\mathrm{TsOH} \cdot \mathrm{H}_{2} \mathrm{O}(8.2 \mathrm{mg}, 0.043 \mathrm{mmol})$ was evacuated in a Schlenk flask for $30 \mathrm{~min}$. Subsequently, methanol $(0.5 \mathrm{~mL})$ was added under $\mathrm{N}_{2}$, and then the Schlenk vessel was transferred to an oil bath at $55-60{ }^{\circ} \mathrm{C}$. The progress of the reaction, for which it took between 2-3 days to complete, was determined by TLC (silica, ether/hexanes); the di-condensation product had $R_{f} \approx 0$ in acetone and in acetonitrile. The crude was then washed with water $(2 \times 2 \mathrm{~mL})$, ethanol containing a drop of $\mathrm{Et}_{3} \mathrm{~N}(1 \times 1 \mathrm{~mL})$, and ether $(3 \times 1 \mathrm{~mL})$, to give bis(1,3-dihydroimidazolidine) 7 as a white solid $(0.115 \mathrm{~g}, 88 \%)$. From another reaction carried out on $0.153 \mathrm{~g}$ of the dialdehyde $\mathbf{5}$, bis(1,3-dihydroimidazolidine) 7 $(0.165 \mathrm{~g}, 90 \%)$ was obtained. ${ }^{1} \mathrm{H}$ NMR $\left(600 \mathrm{MHz}, \mathrm{DMSO}-d_{6}\right): \delta$ 7.706 (s, 4H, OH, exch D O), 7.292 (s, 4H, ArH), 7.160 (s, 4H, $\mathrm{ArH}), 4.444$ (s, 4H, CH), 3.818, $3.796(\mathrm{AB}, J=16.2 \mathrm{~Hz}, 8 \mathrm{H}$, $\left.\mathrm{ArCH}_{2}\right), 3.597\left(\mathrm{t}, J=6.6 \mathrm{~Hz}, 8 \mathrm{H}, \mathrm{OCH}_{2}\right), 3.329\left(\mathrm{~s}, 6 \mathrm{H}, \mathrm{OCH}_{3}\right)$, 3.16-3.14 (br m, 4H, OCH $\left.H_{2}\right), 3.085\left(\mathrm{~s}, 6 \mathrm{H}, \mathrm{OCH}_{3}\right), 3.024(\mathrm{t}, J$ $\left.=7.2 \mathrm{~Hz}, 4 \mathrm{H}, \mathrm{OCH}_{2}\right), 1.053\left(\mathrm{~s}, 6 \mathrm{H}, \mathrm{CH}_{3}\right), 1.007\left(\mathrm{~s}, 6 \mathrm{H}, \mathrm{CH}_{3}\right)$. ${ }^{13} \mathrm{C}$ NMR (150 MHz, DMSO- $\left.d_{6}\right): 155.8,155.0,136.3,135.9$, $132.7,131.5,128.9,113.7,89.8,71.3,70.6,69.7,67.3,66.0$, 58.5, 58.3, 36.6, 24.6, 17.2. LR-FABMS (3-NBA matrix) $m / z$ : calculated for $\mathrm{C}_{54} \mathrm{H}_{75} \mathrm{Br}_{2} \mathrm{~N}_{4} \mathrm{O}_{12}[\mathrm{M}+\mathrm{H}]^{+}$1132.0; found 1131.5. IR $\left(\mathrm{ZnSe}, \mathrm{cm}^{-1}\right): 3433,3335,2980,2924,1454,1358,1207$, $1123,1058,1028,845$.

5.11. Nitronyl nitroxide tetraradical 1: 5,11,17,23-tetrakis(1oxyl-3-0x0-4,4,5,5-tetramethylimidazolin-2-yl)-25,26,27,28-tet rakis(methoxyethoxy)calix [4]arene

Tetrakis(1,3-dihydroimidazolidine) 6 (0.225 g, $0.174 \mathrm{mmol})$ was suspended in $\mathrm{CH}_{2} \mathrm{Cl}_{2}(10 \mathrm{~mL})$, and the suspension was cooled to a temperature between -5 and $0{ }^{\circ} \mathrm{C}$. Subsequently, a solution of $\mathrm{NaIO}_{4}(0.150 \mathrm{~g}, 0.696 \mathrm{mmol})$ in distilled water $(15 \mathrm{~mL})$ was added. The very light blue reaction mixture was stirred at -5 to $0{ }^{\circ} \mathrm{C}$ for $35 \mathrm{~min}$, and then at room temperature for an additional $30 \mathrm{~min}$, resulting in a dark blue reaction mixture. The completion of reaction was determined by TLC (silica, ethyl acetate/acetone), as indicated by the disappearance of starting material and the appearance of a blue spot for tetraradical $\mathbf{1}$. The work up was carried out by the addition of $\mathrm{CH}_{2} \mathrm{Cl}_{2}(40 \mathrm{~mL})$ and then washing of the organic layer with water $(3 \times 30 \mathrm{~mL})$. The organic layer was then dried over $\mathrm{MgSO}_{4}$, filtered, evaporated, and then flash chromatographed (silica, 10-20\% acetone in ethyl acetate) to give $1(80.1 \mathrm{mg})$. Further purification by crystallization from benzene/heptane gave $63.3 \mathrm{mg}$ ( $28 \%$ from two crops) of $\mathbf{1}$. Another reaction, which was carried out at room temperature using $65.4 \mathrm{mg}$ of $\mathbf{6}$, gave tetraradical 1 (15.0 mg, 23\%), after two successive recrystallizations. $\mathrm{Mp} \sim 200{ }^{\circ} \mathrm{C}$ (under $\mathrm{N}_{2}$, dec). ${ }^{1} \mathrm{H}$ NMR (600 MHz, $\left.\mathrm{CDCl}_{3}, 68 \mathrm{mM}\right): \delta 22.5$ (v. br s, 8H, $\mathrm{ArH}$ ), 5.63 (br s, 12H), 4.09 (br s, 24H), -13.6 (v. br s, $\left.48 \mathrm{H}, \mathrm{C}\left(\mathrm{CH}_{3}\right)_{2}\right)$. LR-FABMS (3-NBA matrix) cluster: $\mathrm{m} / z$ (\% RA for $\mathrm{m} / \mathrm{z}=500$ $2000)$ at $(\mathrm{M})^{+} 1277.6(13), 1278.6(15), 1279.6(17), 1280.6(15)$, $1281.6(8)$, at $(\mathrm{M}-\mathrm{O})^{+} 1261.5(25), 1262.5$ (36), 1263.5 (31), 1264.5 (22), $1265.5(13)$, at $(\mathrm{M}-2 \mathrm{O})^{+} 1245.6(25), 1246.5$ (68), 
$1247.5(65), 1248.5(42), 1249.5(26)$, at $(\mathrm{M}-3 \mathrm{O})^{+} 1230.5(88)$, $1231.5(100), 1232.5(70), 1233.5(38)$, at $(\mathrm{M}-4 \mathrm{O})^{+} 1212.5(14)$, 1213.5 (27), 1214.5 (68), 1215.5 (85), 1216.5 (72), 1217.5 (41), $1218.5(17)$, at $(\mathrm{M}-5 \mathrm{O})^{+} 1197.5$ (12), 1198.5 (22), 1199.5 (28), 1200.5 (26), 1201.5 (17). HR-FABMS (3-NBA matrix): $\mathrm{m} / z$ (ion type, \% RA in the $m / z 1270-2100$, deviation for the formula) at $1282.7082\left([\mathrm{M}+6]^{+}, 11.6,1.4 \mathrm{ppm}\right.$ for $\left.{ }^{12} \mathrm{C}_{68}{ }^{1} \mathrm{H}_{98}{ }^{14} \mathrm{~N}_{8}{ }^{16} \mathrm{O}_{16}\right)$, $1281.7126\left([\mathrm{M}+5]^{+}, 23.2,8.1 \mathrm{ppm}\right.$ for $\left.{ }^{12} \mathrm{C}_{67}{ }^{13} \mathrm{C}^{1} \mathrm{H}_{97}{ }^{14} \mathrm{~N}_{8}{ }^{16} \mathrm{O}_{16}\right)$, $1280.7063\left([\mathrm{M}+4]^{+}, 42.8,9.3 \mathrm{ppm}\right.$ for $\left.{ }^{12} \mathrm{C}_{68}{ }^{1} \mathrm{H}_{97}{ }^{14} \mathrm{~N}_{8}{ }^{16} \mathrm{O}_{16}\right)$, $1279.6958\left([\mathrm{M}+3]^{+}, \quad 45.9,7.2 \mathrm{ppm}\right.$ for $\left.{ }^{12} \mathrm{C}_{68}{ }^{1} \mathrm{H}_{95}{ }^{14} \mathrm{~N}_{8}{ }^{16} \mathrm{O}_{16}\right)$, $1278.6907\left([\mathrm{M}+2]^{+}, 45.3,9.3 \mathrm{ppm}\right.$ for $\left.{ }^{12} \mathrm{C}_{68}{ }^{1} \mathrm{H}_{94}{ }^{14} \mathrm{~N}_{8}{ }^{16} \mathrm{O}_{16}\right)$, $1277.6826\left([\mathrm{M}+1]^{+}, 41.4,9.1 \mathrm{ppm}\right.$ for $\left.{ }^{12} \mathrm{C}_{68}{ }^{1} \mathrm{H}_{93}{ }^{14} \mathrm{~N}_{8}{ }^{16} \mathrm{O}_{16}\right)$, $1276.6679\left([\mathrm{M}]^{+}, 16.6\right.$, 3.8 ppm for $\left.{ }^{12} \mathrm{C}_{68}{ }^{1} \mathrm{H}_{92}{ }^{14} \mathrm{~N}_{8}{ }^{16} \mathrm{O}_{16}\right)$. IR (ZnSe, $\left.\mathrm{cm}^{-1}\right): 2981,2925,1452,1386,1356,1218,1125$. Evans method (two measurements, $\mathrm{CDCl}_{3} / \mathrm{CHCl}_{3}, 295$ or $296 \mathrm{~K}$ ), $\chi T=$ 1.3-1.5 emu K mol${ }^{-1}$ (3.5, 4.1 unpaired electrons).

\subsection{Nitronyl nitroxide diradical 2 : 5,17 -bis(1-oxyl-3- oxo-4,4,5,5-tetramethylimidazolin-2-yl)-11,23-dibromo- 25,26,27,28-tetrakis(methoxyethoxy)calix[4]arene}

Bis(1,3-dihydroimidazolidine) 7 (0.160 g, $0.141 \mathrm{mmol})$ was suspended in $\mathrm{CH}_{2} \mathrm{Cl}_{2}(15 \mathrm{~mL})$, and then the suspension was cooled to a temperature between -5 and $0{ }^{\circ} \mathrm{C}$. Subsequently, a solution of $\mathrm{NaIO}_{4}(61.7 \mathrm{mg}, 0.288 \mathrm{mmol})$ in distilled water $(8 \mathrm{~mL})$ was added. The very light blue reaction mixture was stirred at -5 to $0{ }^{\circ} \mathrm{C}$ for $35 \mathrm{~min}$. After the reaction mixture was warmed to $0{ }^{\circ} \mathrm{C}$ and stirred for $15 \mathrm{~min}$ at $0{ }^{\circ} \mathrm{C}$, the color changed to dark blue. The completion of reaction was determined by TLC (silica, ethyl acetate/ether), as indicated by the disappearance of starting material and appearance of a blue spot for the product 2 . For the work up, the reaction mixture was diluted with additional $\mathrm{CH}_{2} \mathrm{Cl}_{2}(40 \mathrm{~mL})$ and then the organic layer was washed with water $(3 \times 30 \mathrm{~mL})$. After drying over $\mathrm{MgSO}_{4}$, filtration, and concentration under vacuo, the crude was purified by flash chromatography (silica, 50\% ethyl acetate in ether), to give diradical $2(89.2 \mathrm{mg})$. The subsequent recrystallization from ethyl acetate $/ \mathrm{CH}_{2} \mathrm{Cl}_{2}$ provided the final diradical product 2 (66.6 mg, 42\% from two crops). From other reactions carried out on 44.8 and $31.2 \mathrm{mg}$ of 7, $21.8 \mathrm{mg}$ (49\% from three crops after recrystallization) and $19.4 \mathrm{mg}$ (63\% from two crops after recrystallization) of $\mathbf{2}$ were obtained, respectively. $\mathrm{Mp} \sim 200{ }^{\circ} \mathrm{C}$ (under $\mathrm{N}_{2}$, dec). ${ }^{1} \mathrm{H}$ NMR (400/600 MHz, $\mathrm{CDCl}_{3}$ ): $\delta 19.5$ (br s, 4H, ArH), 7.280 (s, 4H, ArH), 6.32, 4.874, 4.18 (br s, s, br s, 22H), 3.412 (s, 8H), 3.207 (s, 6H), -14.3 (br s, 24H, $\left.\mathrm{C}\left(\mathrm{CH}_{3}\right)_{2}\right)$. LR-FABMS (3-NBA matrix) cluster: $\mathrm{m} / \mathrm{z}$ (\% RA for $m / z=1000-2000)$ at $(\mathrm{M})^{+} 1123.2(34), 1124.2(45), 1125.2(65)$, 1126.2 (54), 1127.2 (49), $1128.2(36)$, at $(\mathrm{M}-\mathrm{O})^{+} 1107.2$ (34), 1108.2 (45), 1109.2 (64), 1110.2 (73), 1111.2 (54), 1112.2 (42), and at $(\mathrm{M}-2 \mathrm{O})^{+} 1092.1(51), 1093.1$ (67), 1094.1 (100), 1095.1 (93), 1096.1 (73), 1096.1 (49). HR-FABMS (3-NBA matrix): $\mathrm{m} / z$ (ion type, \% RA in the $m / z 1120-2000$, deviation for the formula) at $1128.3372\left([\mathrm{M}+6]^{+}, 29.3,5.0 \mathrm{ppm}\right.$ for ${ }^{12} \mathrm{C}_{54}{ }^{1} \mathrm{H}_{70}{ }^{14} \mathrm{~N}_{4}{ }^{16} \mathrm{O}_{12}{ }^{81} \mathrm{Br}_{2}$ ), $1127.3312\left([\mathrm{M}+5]^{+}, 49.0,5.1 \mathrm{ppm}\right.$ for ${ }^{12} \mathrm{C}_{53}{ }^{13} \mathrm{C}^{1} \mathrm{H}_{70}{ }^{14} \mathrm{~N}_{4}{ }^{16} \mathrm{O}_{12}{ }^{79}$ $\left.\mathrm{Br}^{81} \mathrm{Br}\right), 1126.3340\left([\mathrm{M}+4]^{+}, 61.1,0.3 \mathrm{ppm}\right.$ for ${ }^{12} \mathrm{C}_{54}{ }^{1} \mathrm{H}_{70}{ }^{14} \mathrm{~N}_{4}^{16} \mathrm{O}$ $\left.{ }^{79} \mathrm{Br}^{81} \mathrm{Br}\right), 1125.3293\left([\mathrm{M}+3]^{+}, 70.2,3.1 \mathrm{ppm}\right.$ for ${ }^{12} \mathrm{C}_{54}{ }_{1}^{1} \mathrm{H}_{69}{ }^{4} \mathrm{~N}_{4}$ $\left.{ }^{16} \mathrm{O}_{12}{ }^{81} \mathrm{Br}^{79} \mathrm{Br}\right), 1124.3314\left([\mathrm{M}+2]^{+}, 39.1,0.2 \mathrm{ppm}\right.$ for ${ }^{12} \mathrm{C}_{53}{ }^{13} \mathrm{C}^{1}$ $\left.\mathrm{H}_{69}{ }^{14} \mathrm{~N}_{4}{ }^{16} \mathrm{O}_{12}{ }^{79} \mathrm{Br}_{2}\right), 1123.3290\left([\mathrm{M}+1]^{+}, 32.6,1.0 \mathrm{ppm}\right.$ for ${ }^{12} \mathrm{C}_{54}{ }^{1}$ $\left.\mathrm{H}_{69}{ }^{14} \mathrm{~N}_{4}{ }^{16} \mathrm{O}_{12}{ }^{79} \mathrm{Br}_{2}\right), 1122.3238\left([\mathrm{M}]^{+}, 9.9,3.4 \mathrm{ppm}\right.$ for ${ }^{12} \mathrm{C}_{54}{ }^{1} \mathrm{H}_{68}$ $\left.{ }^{14} \mathrm{~N}_{4}{ }^{16} \mathrm{O}_{12}{ }^{79} \mathrm{Br}_{2}\right)$. IR $\left(\mathrm{ZnSe}, \mathrm{cm}^{-1}\right): 2983,2922,2875,1452,1387$,
1357, 1127. Evans method (one measurement, $\mathrm{CDCl}_{3} / \mathrm{CHCl}_{3}$, $296 \mathrm{~K}), \chi T=0.79 \mathrm{emu} \mathrm{K} \mathrm{mol}{ }^{-1}$ (2.1 unpaired electrons).

\section{Acknowledgements}

This research was supported by the National Science Foundation (CHE-0414936), including the purchase of the Electron Paramagnetic Resonance (EPR) spectrometer (DMR-0216788). Part of this research was performed in facilities renovated with support from NIH (RR16544-01). ChemMatCARS Sector 15 is principally supported by the National Science Foundation/Department of Energy under grant number CHE-0087817. The Advanced Photon Source is supported by the U.S. Department of Energy, Basic Energy Sciences, Office of Science, under Contract No. W-31-109-Eng-38. We thank Dr. Kouichi Shiraishi for help with the EPR spectroscopy and preparation of samples for SQUID magnetometry.

\section{References and notes}

1 A. Rajca, S. Rajca and R. Padmakumar, Angew. Chem., Int. Ed. Engl. 33 (1994), pp. 2091-2093.

2 A. Rajca, Chem. Rev. 94 (1994), pp. 871-893.

3 A. Rajca, Chem.-Eur. J. 8 (2002), pp. 4834-4841.

4 A. Rajca, Adv. Phys. Org. Chem. 40 (2005), pp. 153-199.

5 D.A. Dougherty, Acc. Chem. Res. 24 (1991), pp. 88-94.

6 H. Iwamura and N. Koga, Acc. Chem. Res. 26 (1993), pp. 346-351.

7 W.T. Borden, H. Iwamura and J.A. Berson, Acc. Chem. Res. 27 (1994), pp. 109-116.

8 S. Fang, M.-S. Lee, D.A. Hrovat and W.A. Borden, J. Am. Chem. Soc. 117 (1995), pp. 6727-6731.

9 A. Rajca and S. Rajca, J. Chem. Soc., Perkin Trans. 2 (1998), pp. 1077-1082.

10 In: P.M. Lahti, Editor, Magnetic Properties of Organic Materials, Marcel Dekker, New York, NY (1999), pp. 1-713.

11 In: K. Itoh and M. Kinoshita, Editors, Molecular Magnetism, Gordon and Breach, Amsterdam (2000), pp. 1-337.

12 A. Rajca, S. Rajca and S.R. Desai, J. Am. Chem. Soc. 117 (1995), pp. 806-816.

13 A. Rajca, K. Lu and S. Rajca, J. Am. Chem. Soc. 119 (1997), pp. 10335-10345.

14 A. Rajca, J. Wongsriratanakul and S. Rajca, J. Am. Chem. Soc. 119 (1997), pp. 11674-11686.

15 A. Rajca, S. Rajca and J. Wongsriratanakul, J. Am. Chem. Soc. 121 (1999), pp. 6308-6309.

16 A. Rajca, J. Wongsriratanakul and S. Rajca, Science 294 (2001), pp. $1503-1505$.

17 A. Rajca, J. Wongsriratanakul and S. Rajca, J. Am. Chem. Soc. 126 (2004), pp. 6608-6626.

18 S. Rajca, A. Rajca, J. Wongsriratanakul, P. Butler and S. Choi, J. Am. Chem. Soc. 126 (2004), pp. 6972-6986.

19 A. Rajca, J. Wongsriratanakul, S. Rajca and R.L. Cerny, Chem.-Eur. J. 10 (2004), pp. 3144-3157.

20 G. Ulrich, P. Turek and R. Ziessel, Tetrahedron Lett. 37 (1996), pp. $8755-8758$

21 (a)Q. Wang, Y. Li and G. Wu, Chem. Commun. (2002), pp. 1268 1269. (b)K. Sato, T. Sawai, D. Shiomi, T. Takui, Q. Wang, J.S. Wang, Y. Li and G.S. Wu, Synth. Met. (2003), pp. 1197-1198.

22 L. Kröck, A. Shivanyuk, D.B. Goodin and J. Rebek Jr., Chem. Commun. (2004), pp. 272-273. 
23 A. Rajca, M. Pink, T. Rojsajjakul, K. Lu, H. Wang and S. Rajca, $J$. Am. Chem. Soc. 125 (2003), pp. 8534-8538.

24 A. Rajca, S. Mukherjee, M. Pink and S. Rajca, J. Am. Chem. Soc. 118 (2006), pp. 13497-13507.

25 Sato, H.; Kathirvelu, V.; Spagnol, G.; Rajca, S.; Rajca, A.; Eaton, S. S.; Eaton, G. R.; J. Phys. Chem., submitted for publication.

26 C.S. Winalski, S. Shortkroff, R.V. Mulkern, E. Schneider and G.M. Rosen, Magn. Reson. Med. 48 (2002), pp. 965-972.

27 D.J. Lurie and K. Maeder, Adv. Drug Delivery Rev. 57 (2005), pp. $1171-1190$

28 H. Li, G. He, Y. Deng, P. Kuppusamy and J.L. Zweier, Magn. Reson. Med. 55 (2006), pp. 669-675.

29 K. Matsumoto, F. Hyodo, A. Matsumoto, A.P. Koretsky, A.L. Sowers, J.B. Mitchell and M.C. Krishna, Clin. Cancer Res. 12 (2006), pp. 2455-2462.

30 G. Spagnol, K. Shiraishi, S. Rajca and A. Rajca, Chem. Commun. (2005), pp. 5047-5049.

31 E.F. Ullman, J.H. Osiecki, D.G.B. Boocock and R. Darcy, J. Am. Chem. Soc. 94 (1972), pp. 7049-7059.

32 P.W. Kopf, R.W. Kreilick, D.G.B. Boocock and E.F. Ullman, J. Am. Chem. Soc. 92 (1970), pp. 4531-4535.

33 (a)M.S. Davis, K. Morokuma and R.W. Kreilick, J. Am. Chem. Soc. 94 (1972), pp. 5588-5592. (b) Selected ${ }^{1} \mathrm{H}$-hyperfine splittings from ${ }^{1} \mathrm{H}$ NMR spectra of phenyl nitronyl nitroxide: $a \mathrm{H}($ para $)=+0.0382 \mathrm{mT}$ and $a \mathrm{H}($ ortho $)=+0.0446 \mathrm{mT}$.

34 (a)A.R. Forrester, S.P. Hepburn and G. McConnachie, J. Chem. Soc., Perkin Trans. 1 (1974), pp. 2213-2219. (b) Selected ${ }^{1} \mathrm{H}$-hyperfine splittings in nitroxides: $a \mathrm{H}($ para $)=0.20 \mathrm{mT}$ in phenyl-tert-butylnitroxide and $a \mathrm{H}($ ortho $)=0.24 \mathrm{mT}$ in 3,5-dimethyl-4-methoxyphenyltert-butylnitroxide.

35 A. Izuoka, M. Fukada and T. Sugawara, Mol. Cryst. Liq. Cryst. 232 (1993), pp. 103-108.

36 L. Catala, J.L. Moigne, N. Kyritsakas, P. Rey, J.J. Novoa and P. Turek, Chem.-Eur. J. 7 (2001), pp. 2466-2480.

37 W. Verboom, S. Datta and D.N. Reinhoudt, J. Org. Chem. 57 (1992), pp. 5394-5398.

38 Tetraaldehyde 4 was previously prepared in $66 \%$ yield by the $\mathrm{TiCl}_{4}$ catalyzed formylation with 1,1-dichlorodimethyl ether:N. Maulucci, F. De Riccardis, C.B. Botta, A. Casapullo, E. Cressina, M. Fregonese, P. Tecilla and I. Izzo, Chem. Commun. (2005), pp. 1354-1356.

39 S. Shimono, R. Tamura, N. Ikuma, T. Takimoto, N. Kawame, O. Tamada, N. Sakai, H. Matsuura and J. Yamauchi, J. Org. Chem. 69 (2004), pp. 475-481.

40 Crystal data for nitronyl nitroxide tetraradical 1 $2.25 \mathrm{C}_{6} \mathrm{H}_{6} \cdot 0.25 \mathrm{H}_{2} \mathrm{O}$ : blue block, $0.20 \times 0.15 \times 0.12 \mathrm{~mm}, \mathrm{C}_{81.50} \mathrm{H}_{106} \mathrm{~N}_{8} \mathrm{O}_{16.25}, M=1457.74$, monoclinic, $a=19.576(3) \AA, b=31.283(5) \AA, c=27.078(4) \AA, \beta$ $=103.362(16)^{\circ}, V=16,133(4) \AA^{3}, T=130(2) \mathrm{K}$, space group $P 2_{1} /$ $n, Z=8, \rho_{\text {calcd }}=1.200 \mathrm{Mg} \mathrm{m}^{-3}, \mu=0.084 \mathrm{~mm}^{-1}, 2 \theta_{\max }=45.06$, Mo $\mathrm{K} \alpha(\lambda=0.71073)$. A total of 143,076 reflections were measured, of which 28,452 were independent $\left(R_{\text {int }}=0.0777\right)$. Final residuals were $R=0.1219$ and $w R 2=0.3298$ (for 16,449 observed reflections with $I$ $>2 \sigma(I), 1776$ parameters, 210 restraints) with $\mathrm{GOF}=2.155$ and largest residual peak $1.207 \mathrm{e}^{-3}$ and hole $-0.970 \mathrm{e}^{-3}$. Crystallographic data (excluding structure factors) for the structure reported in this paper have been deposited with the Cambridge Crystallographic Data Centre as supplementary publication no. CCDC-646077.

41. (a) The $\mathrm{C} \cdots \mathrm{C}$ distances in $\AA$ between co-facial ONCNO groups in tetraradical 1: $\mathrm{C} 29 \mathrm{~A} \cdots \mathrm{C} 49 \mathrm{~A}=6.680(7), \mathrm{C} 39 \mathrm{~A} \cdots \mathrm{C} 59 \mathrm{~A}=6.893(7)$, C29B...C49B $=7.132(7)$, and C39B $\cdots C$ C59B $=6.961(8)$. These $C \cdots C$ distances are relatively accurate, as they are not affected by any of the disorders, including disorders in side chains and in solvent molecules, as well as the rotational disorder of imidazoline rings; (b) The $\mathrm{C} \cdots \mathrm{C}$ distances in $\AA$ between co-facial ONCNO groups in diradical 2: $\mathrm{C} 32 \mathrm{a} \cdots \mathrm{C} 45 \mathrm{a}=7.73(3)$ and $\mathrm{C} 32 \mathrm{~b} \cdots \mathrm{C} 45 \mathrm{~b}=7.70(3)$; (c) The $(\mathrm{C} 1-\mathrm{C} 6)-(\mathrm{C} 15-\mathrm{C} 20)$ and $(\mathrm{C} 8-\mathrm{C} 13)-(\mathrm{C} 22-\mathrm{C} 27)$ dihedral angles between calculated least-squares planes of the co-facial benzene rings in $\mathbf{1}, \mathbf{2}$, and the corresponding nitroxide tetraradical and diradical are as follows: in $1,19.74(9)^{\circ}, 25.45(22)^{\circ}, 22.87(12)^{\circ}, 24.05(22)^{\circ}$; in $2,36.2(5)^{\circ}, 38.9(9)^{\circ}, 36.7(9)^{\circ}, 36.3(9)^{\circ}$; in nitroxide tetraradical, 3.62(7) ${ }^{\circ}, 2.85(7)^{\circ}$; in nitroxide diradical, 3.38(19) ${ }^{\circ}, 13.96(17)^{\circ}$, $8.42(9)^{\circ}, 13.80(18)^{\circ}$

42 R.R. Sharp, Nucl. Magn. Reson. 34 (2005), pp. 553-596.

43 (a)D.F. Evans, J. Chem. Soc. (1959), pp. 2003-2005. (b)D.H. Live and S.I. Chan, Anal. Chem. 42 (1970), pp. 791-792.

44 Two types of protons (AB or AX spin system) are expected for the methylene groups of the macrocycle in diradical 2. An AB spin system is observed for the corresponding protons in bis(1,3-dihydroimidazolidine) 7 in DMSO- $d_{6}$ at room temperature.

45 The numerical fits with two components, tetraradical and triradical for $\mathbf{1}$, and diradical and monoradical for $\mathbf{2}$, suggest $99+\%$ content of tetraradical and diradical, respectively.

46 2-(4'-Hydroxyphenyl)-4,4,5,5-tetramethylimidazoline-1-oxyl-3-oxide and related nitronyl nitroxide radicals, see: Ref. 31.

47 In the point-dipole approximation, $|D / h c|$ is related to $1 / r^{3}$, where $r$ is the radical-radical distance.

48 Very small value of mean-field parameter, $\theta \approx-0.1 \mathrm{~K}$, is also obtained for $5 \mathrm{mM} 2$ in chloroform.

49 A. Rajca, S. Rajca and J. Wongsriratanakul, Chem. Commun. (2000), pp. 1021-1022.

50 N.L. Frank, R. Clérac, J.-P. Sutter, N. Daro, O. Kahn, C. Coulon, M.T. Green, S. Golhen and L. Ouahab, J. Am. Chem. Soc. 122 (2000), pp. 2053-2061.

51 In addition to intermolecular radical-radical $\mathrm{O} \cdots \mathrm{O}$ distances of 4.5 $6.1 \AA$, network of short intermolecular radical-radical $\mathrm{O} \cdots \mathrm{C}$ distances may be identified. For example, the O1-C6(meta) contacts at $3.442 \AA$ are between aryl nitronyl nitroxide moieties in the adjacent spiroconjugated diradical molecules, thus effectively forming dimers, i.e., antiferromagnetically coupled pairs of $S=1 / 2$ spins. The presence of two O1-C6(meta) contacts per pair, which may be viewed as two parallel exchange coupling pathways, will further amplify the strength of the antiferromagnetic coupling (Ref. 49).

52 Microwave saturation may have a significant effect when $\left|\Delta m_{\mathrm{s}}\right|=1$ transitions are used for the intensity versus $1 / T$ plots near the liquid helium temperature range. Similar artefacts, due to saturation, are likely for very weak $\left|\Delta m_{\mathrm{s}}\right|=2$ transitions in diradicals with small values of $|D / h c|$.

53 (a)A. Rajca and S. Rajca, J. Am. Chem. Soc. 118 (1996), pp. 81218126. (b) A. Rajca, K. Lu, S. Rajca and C.R. Ross II, Chem. Commun. (1999), pp. 1249-1250.

54 J. Suffert, J. Org. Chem. 54 (1989), pp. 509-510.

55 D.D. Perrin and W.L.F. Armarego, Purification of Laboratory Chemicals (3rd ed.), Pergamon, Oxford (1988) p 365.

56 R.L. Carlin, Magnetochemistry, Springer, Berlin (1986) pp 2-4.

\section{Supplementary data}

General procedures and materials, additional crystallographic data for $\mathbf{1}$ and 2, SQUID magnetometry data for diradical $\mathbf{2}$ in chloroform, preparation of 2,3-bis(hydroxyamino)-2,3-dimethylbutane and nitronyl nitroxide monoradicals, IR, ${ }^{1} \mathrm{H}$ NMR and ${ }^{13} \mathrm{C}$ NMR spectra for selected compounds $1-7$. 


\title{
Supplementary Data
}

1,3-Alternate Calix[4]arene Nitronyl Nitroxide Tetraradical and Diradical:

Synthesis, X-ray Crystallography, Paramagnetic NMR Spectroscopy, EPR Spectroscopy, and Magnetic Studies.

\author{
Andrzej Rajca*, ${ }^{a}$ Maren Pink, ${ }^{b}$ Sumit Mukherjee, ${ }^{a}$ Suchada Rajca, ${ }^{a}$ Kausik Das ${ }^{a}$ \\ Department of Chemistry, University of Nebraska, Lincoln, Nebraska 68588-0304. IUMSC, \\ Department of Chemistry, Indiana University, Bloomington, Indiana 47405-7102. \\ E-mail address: arajca1@unl.edu
}

${ }^{\mathrm{a}}$ University of Nebraska.

${ }^{\mathrm{b}}$ Indiana University. 


\section{Table of Contents}

1. X-ray Crystallography (Tables S1 and S2).

2. Preparation of 2,3-Bis(hydroxyamino)-2,3-dimethylbutane and Nitronyl Nitroxide Monoradicals.

3. Acknowledgment.

4. References for Supporting Information.

5. Figures S1 - S18.

(a) Plots for tetraradical 1 and diradical 2, and crystal packing of 2 (Figures S1, S2, and S3).

(b) ${ }^{1}$ H NMR spectra of 1 and 2 (Figures S4 and S5)

(c) SQUID magnetometry data for 2 in chloroform (Figure S6).

(d) IR spectra for nitronyl nitroxide radicals and corresponding 1,3dihydroimidazolidines: 1, 2, 6, and 7 (Figures S7 - S10).

(e) ${ }^{1} \mathrm{H}$ and ${ }^{13} \mathrm{C}$ NMR spectra for synthetic intermediates 4, 5, 6, and 7 (Figures S11 S16).

(f) ${ }^{1}$ H NMR spectra for selected nitronyl nitroxide monoradicals (Figures S17 and S18). 


\section{X-ray Crystallography.}

Data collection, structure solution and refinement. Data collections were carried out using Mo Ka radiation $(\lambda=0.71073 \AA$, graphite monochromator $)$ at $130 \mathrm{~K}$ and synchrotron radiation $(\lambda=0.49594 \AA)$ at $100 \mathrm{~K}$ for $\mathbf{1}$ and $\mathbf{2}$, respectively. The intensity data were corrected for absorption. ${ }^{\mathrm{S} 1}$ Final cell constants were calculated from the xyz centroids of strong reflections from the actual data collection after integration. $^{\text {S2 }}$

Space groups were determined based on intensity statistics and systematic absences. Structures were solved with direct methods using SIR-2004 ${ }^{\mathrm{S} 3}$ and refined with full-matrix least squares / difference Fourier cycles using SHELXL-97. ${ }^{\text {S4 }}$

For tetraradical 1, all non-hydrogen atoms were refined with anisotropic displacement parameters except for some carbon and oxygen atoms in multiply disordered methoxyethylene chains and nonhydrogen atoms in solvent molecules (benzene and water). The hydrogen atoms were placed in ideal positions and refined as riding atoms with relative isotropic displacement parameters; the hydrogen atoms of a partial water ware placed based on possible hydrogen bonding. The diffraction pattern of $\mathbf{1}$ was of low quality, with streaks and diffuse scattering indicating a highly disordered structure. Diffraction rapidly weakens at medium and high resolution; in fact diffraction terminates at $2 \theta$ angles of about $45^{\circ}$. Accordingly, the obtained structure is of low quality. Numerous restraints and constraints were applied to the disordered parts of the molecule. These include rigid-bond and similar-bond restraints, restrained anisotropic refinements using displacement parameter restraints, rigid body constraints, anti-bumping restraints, fixed partial occupancies, and others. The calixarene molecule is very flexible in that the methoxyethylene chains can adopt a variety of conformations and the imidazoline ring can rotate about the bond to the calixarene frame. Inefficient packing of the molecules (layer structure with two independent calixarene molecules and benzene solvent molecules in channels along $[1 / 2,0,1 / 2]$ and between the layers) allow for extensive disorder of both the five-membered rings and the methoxyethylene chains. 
For diradical 2, several attempts to obtain sufficient data at a conventional diffractometer failed. Data were collected from a tiny, dry, aged crystal using synchrotron radiation. The diffraction pattern shows diffuse scattering and terminated at low resolution (presumably because of solvent and crystallinity loss over time and because of a highly disordered structure), thus giving rise to a weak, marginal dataset. A preliminary structure however, could be obtained, which established the connectivity and overall geometry of the compound. All atoms remained isotropic and hydrogen atoms were included at calculated positions. No disorder was modeled because of the lack of data. Solvent was not modeled in solvent accessible voids and channels in the structure. Soft restraints were used for the methoxyethylene chains, restraining 1-2 and 1-3 distances to be similar.

Nitronyl nitroxide tetraradical 1. The compound crystallizes in space group $\mathrm{P} 2{ }_{1} / \mathrm{n}$ with two molecules of tetraradical, including several (partial) solvent molecules (benzene and water) per asymmetric unit. The final full matrix least squares refinement converged to $\mathrm{R} 1=0.1219$ and $\mathrm{wR} 2=$ 0.3628 (F2, all data). The remaining electron density was located around disordered methoxyethylene chains.

Nitronyl nitroxide diradical 2. ${ }^{\text {S5 }}$ The compound crystallizes in space group P-1 with an unknown amount of solvent. This preliminary structure was not converged; standard deviations of the geometrical parameters, remaining electron density, and R-values are high. 
Table S1. Selected intramolecular distances $(\AA)$ between the co-facial radical moieties: ONCNO in $\mathbf{1}$ and 2, and NO in the corresponding nitroxide tetraradical and diradical.

\begin{tabular}{|c|c|c|c|}
\hline & $\mathrm{C} \cdots \mathrm{C}$ & $\mathrm{N} \cdots \mathrm{N}$ & $\mathrm{O} \cdots \mathrm{O}$ \\
\hline \multirow{4}{*}{$\begin{array}{l}\text { Nitronyl nitroxide } \\
\text { tetraradical } 1\end{array}$} & $6.680(7)$ & $6.64,6.95,7.10,7.44$ & $6.35,7.27,7.74,8.68$ \\
\hline & $6.893(7)$ & $6.03,7.28,7.31,8.05$ & $4.76,8.07,8.11,9.26$ \\
\hline & $6.961(8)$ & $6.63,7.58,7.82,8.24$ & $5.49,8.18,8.71,9.14$ \\
\hline & $7.132(7)$ & $7.46,7.48,6.76,8.66$ & $6.64,7.31,7.46,10.30$ \\
\hline Nitroxide tetraradical $^{\mathrm{S} 6}$ & - & $5.78,5.70$ & $6.92,6.05$ \\
\hline Nitronyl nitroxide & $7.70(3)$ & $7.19,8.37,8.47,9.21$ & $6.03,8.98,9.27,10.30$ \\
\hline diradical 2 & $7.73(3)$ & $7.08,8.45,8.49,9.47$ & $5.49,8.90,8.92,10.57$ \\
\hline Nitroxide diradical $^{\mathrm{S} 6}$ & - & $5.07,5.37$ & $5.70,5.45$ \\
\hline
\end{tabular}

Table S2. Dihedral angles between the co-facial benzene ring planes for nitronyl nitroxide tetraradical 1 and diradical 2, and the corresponding nitroxide tetraradical and diradical.

\begin{tabular}{|c|c|c|}
\hline & $\begin{array}{c}(\mathrm{C} 1-\mathrm{C} 6)-(\mathrm{C} 15-\mathrm{C} 20) \text { angle } \\
\left.{ }^{\circ}\right]\end{array}$ & $\begin{array}{l}(\mathrm{C} 8-\mathrm{C} 13)-(\mathrm{C} 22-\mathrm{C} 27) \text { angle } \\
{\left[{ }^{\circ}\right]}\end{array}$ \\
\hline Nitronyl nitroxide & Mol A, 19.74(9) & Mol A, 22.9(1) \\
\hline tetraradical 1 & Mol B, 25.4(2) & Mol B, 24.0(2) \\
\hline Nitroxide tetraradical ${ }^{\mathrm{S} 6}$ & $3.62(7)$ & $2.85(7)$ \\
\hline Nitronyl nitroxide & Mol A, 36.2(5) & Mol A, 36.7(9) \\
\hline diradical 2 & Mol B, 38.9(9) & Mol B, 36.3(9) \\
\hline \multirow[t]{2}{*}{ Nitroxide diradical $^{\mathrm{S} 6}$} & Mol A, 3.4(2) & Mol A, 8.42(9) \\
\hline & Mol B, 14.0(2) & Mol B, 13.8(2) \\
\hline
\end{tabular}




\section{Preparation of 2,3-Bis(hydroxyamino)-2,3-dimethylbutane and Nitronyl Nitroxide}

\section{Monoradicals.}

\section{2,3-Bis(hydroxyamino)-2,3-dimethylbutane. ${ }^{\mathrm{S}}$ Aluminum foil $(0.809 \mathrm{~g}, 3.0 \mathrm{mmol})$ was weighed}

and was torn into tiny shreds, before adding a $3 \%$ aqueous solution of $\mathrm{HgCl}_{2}(18.0 \mathrm{~mL}, 0.543 \mathrm{~g}, 2.0$ mmol). Upon stirring the resultant mixture for $2-3 \mathrm{~min}$ at room temperature, it turned black and the aluminum foil pieces were tarnished. The liquid portion was removed with a pipette and the residual solid was successively washed with water, methanol, and THF two times each. After the amalgamated aluminum was covered with THF $(23 \mathrm{~mL})$ and cooled to -8 to $-10{ }^{\circ} \mathrm{C}$, water $(3.5 \mathrm{~mL})$ and a solution of 2,3-dimethyl-2,3-nitrobutane $(1.0 \mathrm{~g}, 5.7 \mathrm{mmol})$ in THF $(20 \mathrm{~mL})$ were successively added. The reaction mixture was stirred between -2 and $-7{ }^{\circ} \mathrm{C}$ for $45 \mathrm{~min}$. The aluminum dissolved to leave a black solution. The reaction mixture was filtered through a narrow pad of Celite and the filtrate was concentrated in vacuo. The residual solid was washed with ether $(2 \times 3 \mathrm{~mL})$ to give $0.587 \mathrm{~g}(70 \%$, sm7-89) of 2,3-bis(hydroxyamino)-2,3-dimethylbutane as a white solid. From other two reactions done on $1.0 \mathrm{~g}$ (sm-8-64) and $100.0 \mathrm{mg}$ ( $\mathrm{sm}-7-18)$ of the dinitro compound, the product was obtained in $75 \%$ and $79 \%\left(\right.$ (lit $^{\mathrm{S} 7} 76 \%$ ) yield respectively. ${ }^{1} \mathrm{H}$ NMR (500 MHz, DMSO- $d_{6}$, sm-7-18DMSO): $\delta=6.923(\mathrm{~s}, 2 \mathrm{H}$, $\mathrm{OH}$, exch $\left.\mathrm{D}_{2} \mathrm{O}\right), 5.379(\mathrm{~s}, 2 \mathrm{H}, \mathrm{NH}), 0.992\left(\mathrm{~s}, 12 \mathrm{H}, \mathrm{CH}_{3}\right){ }^{\mathrm{S} 8}$

\section{1,3-Dihydroxy-2-(4-methoxyphenyl)-4,4,5,5-tetramethylimidazolidine (S1) A mixture of 4-} methoxybenzaldehyde (0.100 g, $0.73 \mathrm{mmol}), 2,3$-bis(hydroxyamino)-2,3-dimethylbutane (0.119 g, $0.803 \mathrm{mmol})$, p-TsOH. $\mathrm{H}_{2} \mathrm{O}(7 \mathrm{mg}, 0.037 \mathrm{mmol})$, and methanol $(\sim 0.5 \mathrm{~mL})$ was stirred under $\mathrm{N}_{2}$ for 2 days at room temperature. The completion of reaction was determined by TLC (silica, ether/chloroform), as indicated by the disappearance of the spot for the starting material and appearance of a more polar spot for the condensation product. The reaction mixture was successively washed with water $(2 \times 1 \mathrm{~mL})$, ethanol containing a drop of $\mathrm{Et}_{3} \mathrm{~N}(1 \times 1 \mathrm{~mL})$, and ether $(2 \times 2 \mathrm{~mL})$ to give imidazolidine S1 as a white solid (148.1 mg, 76\%, sm-7-53). From two other reactions carried out on $15.0 \mathrm{mg}$ (sm-7-34) and $20.0 \mathrm{mg}$ (sm-7-41) of 4-methoxybenzaldehyde, imidazolidine S1 was obtained 
in $87 \%$ yield $\left(\mathrm{lit}^{\mathrm{S} 7} 72 \%\right) .{ }^{1} \mathrm{H}$ NMR (400 MHz, DMSO-d 6 , sm-7-53aniscond-ethwash): $\delta=7.675$ (s, 2H, $\mathrm{OH}$, exch $\left.\mathrm{D}_{2} \mathrm{O}\right), 7.362(\mathrm{~d}, 8.8 \mathrm{~Hz}, 2 \mathrm{H}, \mathrm{ArH}), 6.872$ (d, $\left.8.8 \mathrm{~Hz}, 2 \mathrm{H}, \mathrm{ArH}\right), 4.441(\mathrm{~s}, 1 \mathrm{H}, \mathrm{CH}), 3.732$ (s, $\left.3 \mathrm{H}, \mathrm{CH}_{3}\right), 1.055\left(\mathrm{~s}, 3 \mathrm{H}, \mathrm{CH}_{3}\right), 1.030\left(\mathrm{~s}, 3 \mathrm{H}, \mathrm{CH}_{3}\right)$.

1,3-Dihydroxy-2-phenyl-4,4,5,5-tetramethylimidazolidine (S2). A mixture of benzaldehyde (0.100 g, $0.94 \mathrm{mmol}), 2$,3-bis(hydroxyamino)-2,3-dimethylbutane (0.154 g, $1.04 \mathrm{mmol})$, p-TsOH. $\mathrm{H}_{2} \mathrm{O}$ (9 mg, $0.047 \mathrm{mmol}$ ), and methanol $(\sim 0.5 \mathrm{~mL})$ was stirred under $\mathrm{N}_{2}$ for 2 days at room temperature. The completion of reaction was determined by TLC (silica, ether/chloroform), as indicated by the disappearance of the spot for the starting material and appearance of a more polar spot for the condensation product. The reaction mixture was successively washed with water $(2 \times 1 \mathrm{~mL})$, ethanol containing a drop of $\mathrm{Et}_{3} \mathrm{~N}(1 \times 1 \mathrm{~mL})$, and ether $(2 \times 2 \mathrm{~mL})$ to give the condensation product as a white solid (199.6 mg, 90\%, sm-7-53). In two other reactions carried out on $221.0 \mathrm{mg}(\mathrm{sm}-8-41)$ and $28.6 \mathrm{mg}$ (sm-7-41) of the benzaldehyde, the condensation product was obtained in $96 \%$ and $77 \%$ yield $\left(\right.$ lit $^{\mathrm{S}}$ $82 \%$ ) respectively. ${ }^{1} \mathrm{H}$ NMR (400 MHz, DMSO- $d_{6}$, sm-7-53benzcond-ethwash): $\delta=7.740$ (s, $2 \mathrm{H}, \mathrm{OH}$, exch $\left.\mathrm{D}_{2} \mathrm{O}\right), 7.467$ (d, 7.6 Hz, 2H, ArH), 7.33 - 7.23 (m, 3H, ArH), 4.494 (s, 1H, CH), 1.070 (s, 3H, $\left.\mathrm{CH}_{3}\right), 1.039\left(\mathrm{~s}, 3 \mathrm{H}, \mathrm{CH}_{3}\right)$.

2-(4-Methoxyphenyl)-4,4,5,5-tetramethylimidazoline-1-oxyl-3-oxide (S3). 1,3-Dihydroxy-2-(4methoxyphenyl)-4,4,5,5-tetramethylimidazolidine (0.100 g, $0.37 \mathrm{mmol})$, was suspended in chloroform $(10 \mathrm{~mL})$, and then a solution of $\mathrm{NaIO}_{4}(0.396 \mathrm{~g}, 1.85 \mathrm{mmol})$ in distilled water $(8 \mathrm{~mL})$ was added. The color almost instantly changed from light blue to dark blue. The completion of reaction was determined by TLC (silica, ethyl acetate/hexanes), as indicated by the disappearance of starting material and appearance of a blue spot for the nitronyl nitroxide radical. The reaction mixture was taken up in $20 \mathrm{~mL}$ of additional chloroform and the organic layer was washed with water $(3 \times 10 \mathrm{~mL})$. The organic layer was then dried over $\mathrm{MgSO}_{4}$, filtered, and concentrated in vacuo. The resultant crude was purified by flash chromatography (silica, 10-20\% ethyl acetate in hexane) to give $83.7 \mathrm{mg}$ of nitronyl nitroxide radical. The product was then recrystallized from ethyl acetate/heptane to give $48.2 \mathrm{mg}$ ( $48 \%$ from one 
crop, sm-7-58) of the radical. ${ }^{1} \mathrm{H}$ NMR $\left(400 \mathrm{MHz}, \mathrm{CDCl}_{3}\right.$, sm-7-58anisrecry1): $\delta=+0.744(\mathrm{~s}, 3 \mathrm{H}$, $\left.\mathrm{ArOCH}_{3}\right),-4.825$ (bs, 2H, $\left.\mathrm{ArH}\right),-14.13\left(\mathrm{bs}, 12 \mathrm{H}, \mathrm{CH}_{3}\right)$.

2-Phenyl-4,4,5,5-tetramethylimidazoline-1-oxyl-3-oxide (S4). 1,3-Dihydroxy-2-phenyl-4,4,5,5tetramethylimidazolidine S2 $(0.200 \mathrm{~g}, 0.85 \mathrm{mmol})$ was suspended chloroform $(20 \mathrm{~mL})$. The suspension was cooled to $0{ }^{\circ} \mathrm{C}$, and then a solution of $\mathrm{NaIO}_{4}(0.182 \mathrm{mg}, 0.85 \mathrm{mmol})$ in distilled water $(5 \mathrm{~mL})$ was added. The very light blue reaction mixture was stirred at $0{ }^{\circ} \mathrm{C}$ for $15 \mathrm{~min}$, after which the ice bath was removed and the mixture was allowed to warm to room temperature and stirred for an additional 10 min, during which the color changed to purple. The completion of reaction was determined by TLC (silica, ethyl acetate/hexanes), as indicated by the disappearance of starting material and appearance of a purple spot for the nitronyl nitroxide radical S4. The reaction mixture was taken up in $20 \mathrm{~mL}$ of additional chloroform and the organic layer was washed with water $(3 \times 10 \mathrm{~mL})$. After drying over $\mathrm{MgSO}_{4}$, filtration, and concentration in vacuo, the crude product was obtained as a purple solid. This crude was combined with the crude obtained from another reaction (sm-8-43), carried out on $104.5 \mathrm{mg}$ of S2, and then purified by flash chromatography (silica, 10-20\% ethyl acetate in hexane) to give 264.2 mg of nitronyl nitroxide radical S4. The product was recrystallized from ethyl acetate/heptane, to give $254.2 \mathrm{mg}$ (85\% from three crops, sm-8-46) of pure radical S4. From other reactions carried out on $262.1 \mathrm{mg}$ (sm-8-47) and $100 \mathrm{mg}\left(\mathrm{sm}-7-58,5\right.$ equivalents of $\mathrm{NaIO}_{4}$ was used for oxidation) of S2, 143.5 $\mathrm{mg}$ (56\% from one crop,) and $42.5 \mathrm{mg}$ (43\% from two successive recrystallizations) respectively of nitronyl nitroxide radical S4 was obtained. ${ }^{1} \mathrm{H}$ NMR (400 MHz, $\mathrm{CDCl}_{3}$, sm-7-58benzal-N,Ncol1): $\delta=$ $\sim 38.3$ (br m, 3H, ArH), -5.52 (bs, 2H, ArH), -13.46 (bs, 12H, $\mathrm{CH}_{3}$ ).

\section{Acknowledgment}

The plots in Figure 11 and Figure 12 were prepared using Mercury1.4.1 program from CCDC. 


\section{References for Supporting Information}

S1. SADABS: Blessing, R. Acta Cryst. A 1995, 51, 33-38.

S2. SAINT 6.1, Bruker Analytical X-Ray Systems, Madison, WI, 1999.

S3. SIR-2004, A Program for Atomatic Solution and Refinement of Crystal Structure, Burla, M. C.;

Caliandro, R.; Carnalli, M.; Carrozzini, B.; Cascarano, G. L.; De Caro, L.; Giacovazzo, C.; Polidori, G.; Sagna, R., version 1.0, 2004.

S4. SHELXTL-Plus (current version), Bruker Analytical X-Ray Systems, Madison, WI.

S5. Crystal data for nitronyl nitroxide diradical 2: Blue block, $0.05 \times 0.03 \times 0.02 \mathrm{~mm}$, $\mathrm{C}_{54} \mathrm{H}_{68} \mathrm{Br}_{2} \mathrm{~N}_{4} \mathrm{O}_{12} \cdot n$ solvent, triclinic, $P-1, a=16.22(3) \AA, b=16.23(2) \AA, c=22.64(4) \AA, \alpha=$ $86.50(7)^{\circ}, \beta=76.12(8)^{\circ}, \gamma=71.97(7)^{\circ}, V=5501(17) \AA^{3}, T=100(2) \mathrm{K}, Z=4$.

S6. Rajca, A.; Mukherjee, S.; Pink, M.; Rajca, S. J. Am. Chem. Soc. 2006, 118, 13497 - 13507.

S7. Preparation of 2,3-bis(hydroxyamino)-2,3-dimethylbutane by reduction of dinitrobutane with A1/Hg in aqueous THF: Shimono, S.; Tamura, R.; Ikuma, N.; Takimoto, T.; Kawame, N.; Tamada, O.;

Sakai, N.; Matsuura, H.; Yamauchi, J. J. Org. Chem. 2004, 69, 475 - 481.

S8. ${ }^{1} \mathrm{H}$ NMR (DMSO- $d_{6}$ ) spectrum of 2,3-bis(hydroxyamino)-2,3-dimethylbutane: Wautelet, P.; Moigne, J. L.; Videva, V.; Turek, P. J. Org. Chem. 2003, 68, 8025 - 8036. 


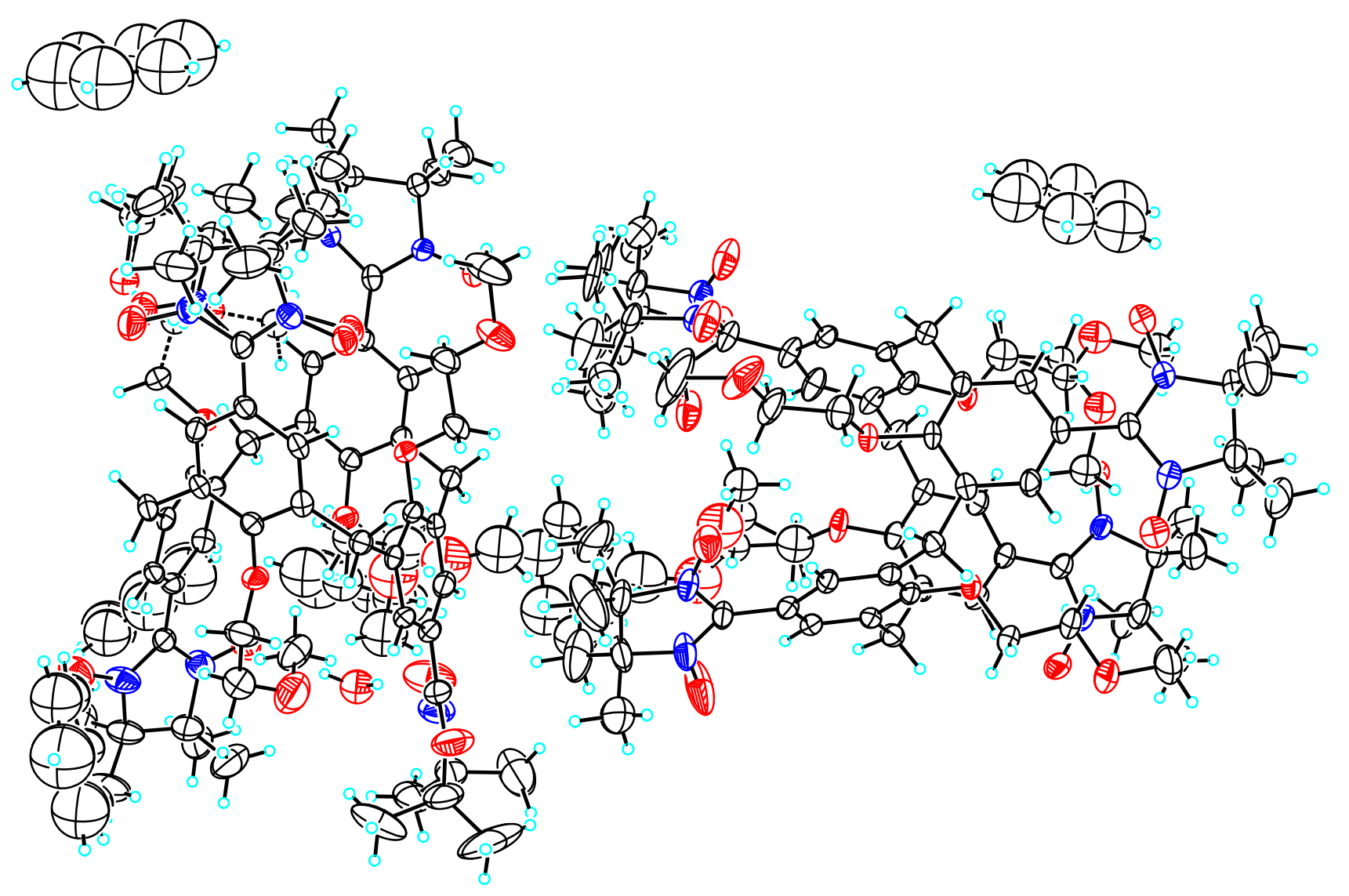

Figure S1. Ortep plot of tetraradical 1: asymmetric unit, all disorder shown. Carbon, nitrogen, and oxygens atoms are depicted with thermal ellipsoids or spheres set at the $50 \%$ probability level. 

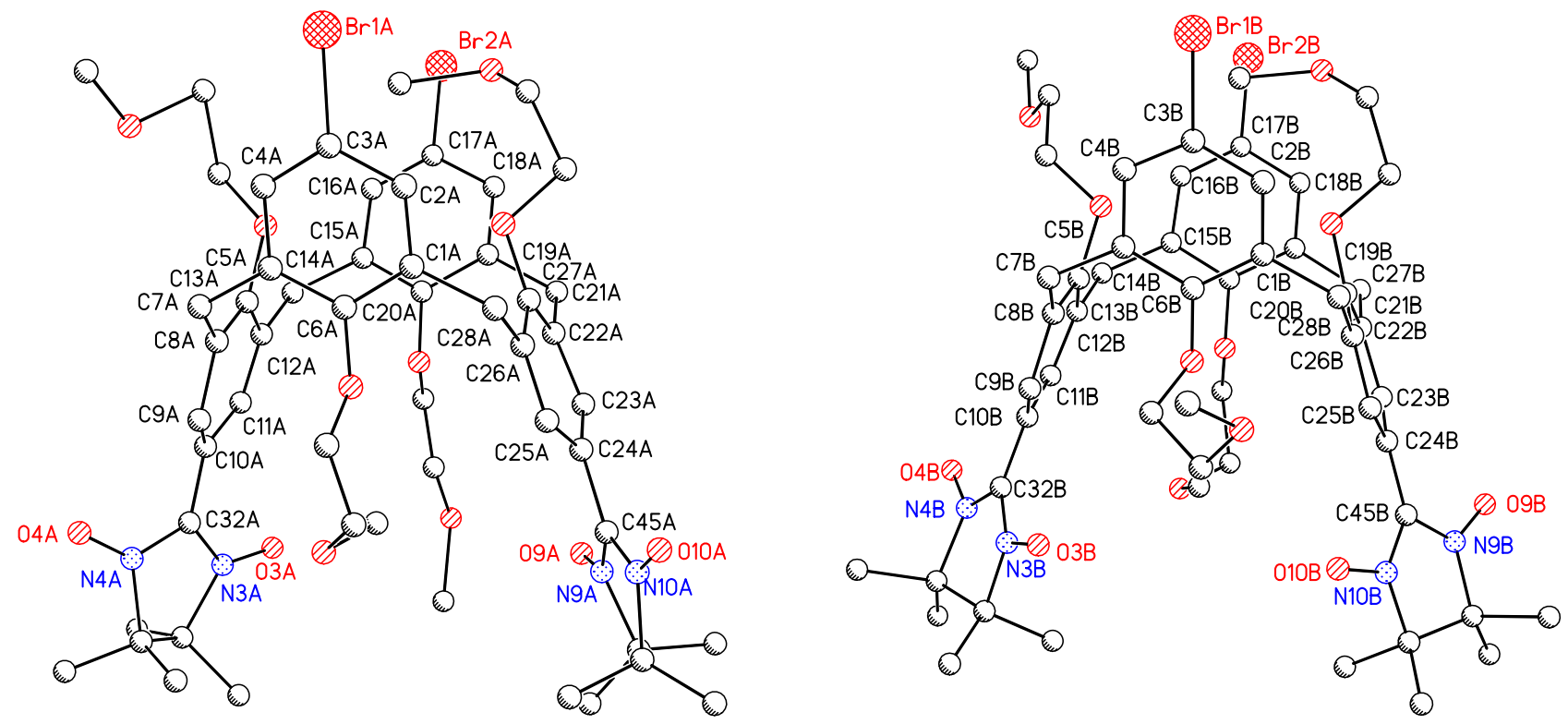

Figure S2. Ball-and-stick model of the independent molecules A and B of diradical 2, side by side. Hydrogen atoms are omitted for clarity; no disorder or solvent were included in this preliminary model.

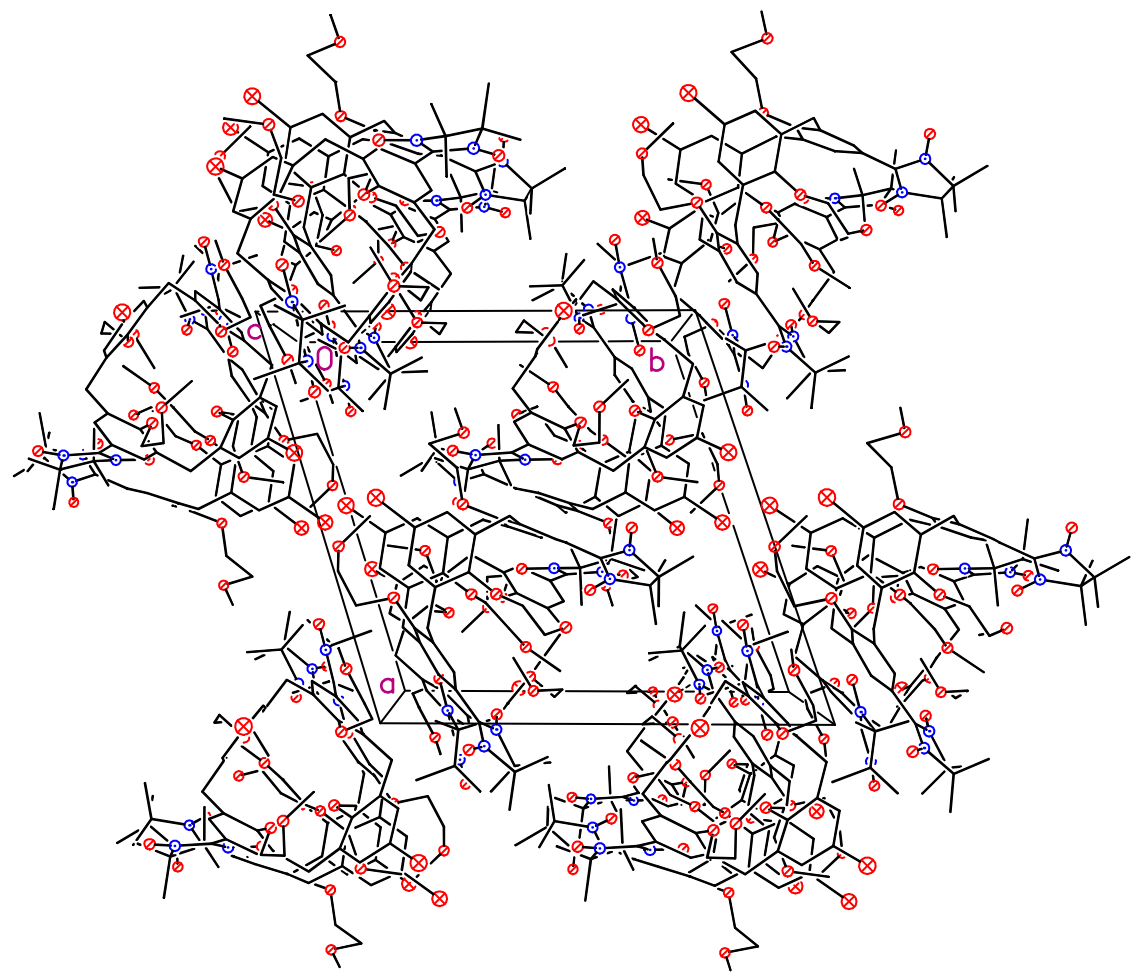

Figure S3a. Crystal packing of diradical 2 with layers along the $\left(\begin{array}{lll}1 & 1 & 0\end{array}\right)$. 


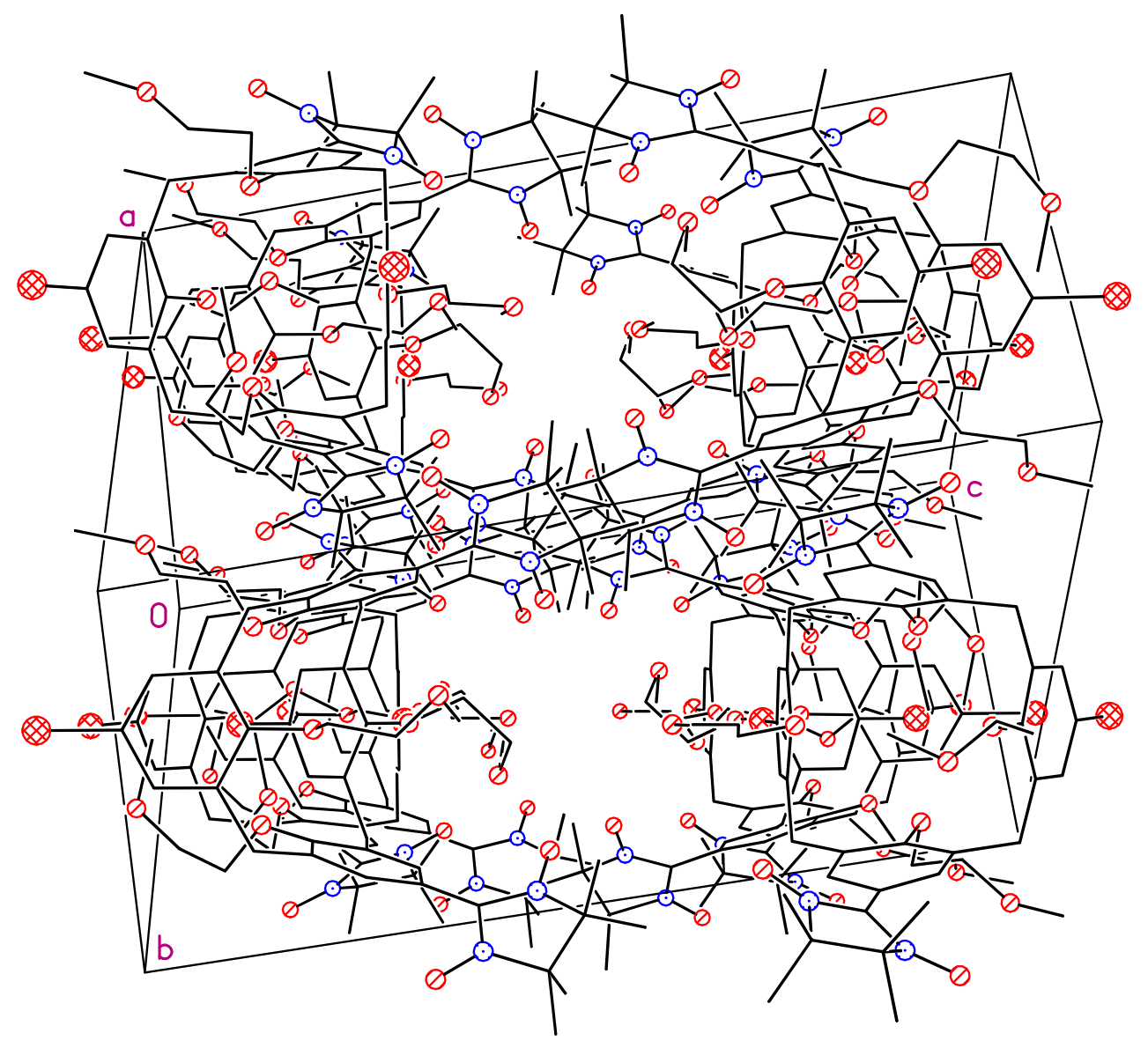

Figure S3b. Crystal packing of diradical 2; top view of ( $\left(\begin{array}{lll}1 & 1 & 0\end{array}\right)$ layer showing solvent accessible channels. 


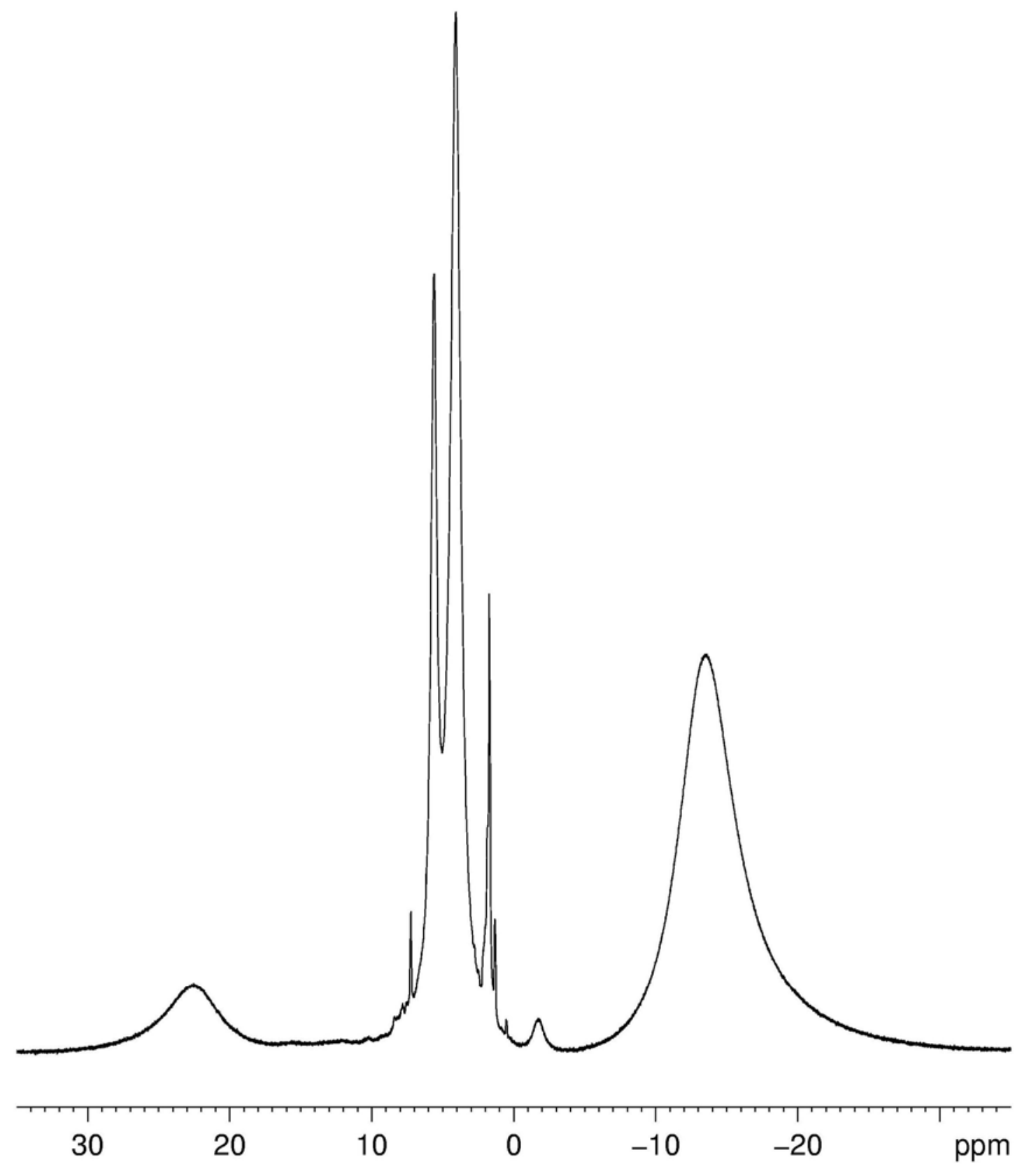

Figure S4. ${ }^{1} \mathrm{H}$ NMR $(600 \mathrm{MHz}, \mathrm{LB}=1 \mathrm{~Hz})$ spectrum of $\sim 70 \mathrm{mM}$ tetraradical 1 in chloroform-d (label: SM-11-19-R1). This is an expanded plot of the spectrum in Figure 5 (main text). 


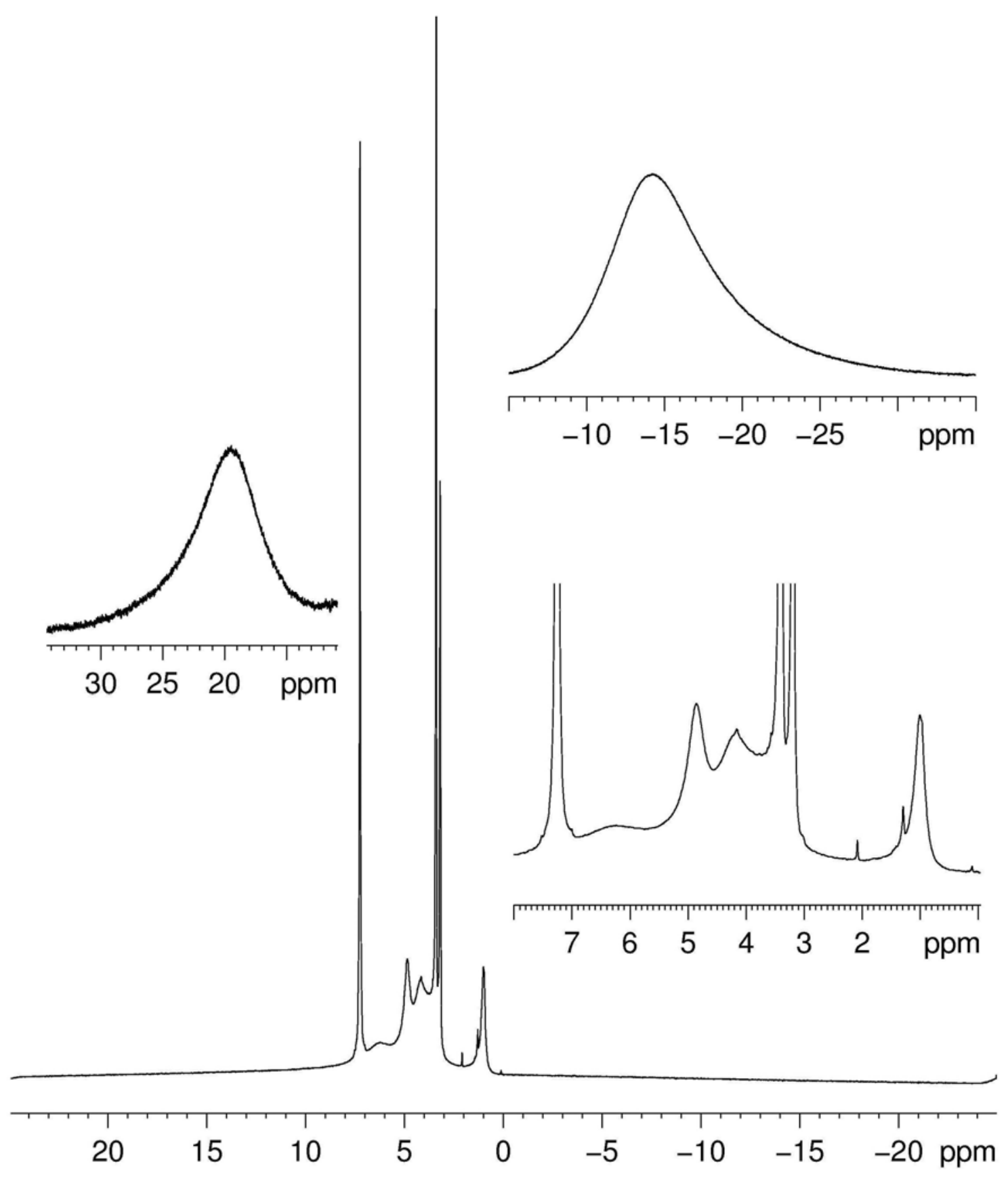

Figure S5. The main plot and the bottom inset: ${ }^{1} \mathrm{H}$ NMR $(400 \mathrm{MHz}, \mathrm{LB}=5 \mathrm{~Hz})$ spectrum of dilute solution of diradical 2 in chloroform-d (label: SM-10-50col1). The two top insets: ${ }^{1} \mathrm{H}$ NMR (600 MHz, $\mathrm{LB}=5 \mathrm{~Hz}$ ) spectrum of concentrated, $\sim 98 \mathrm{mM}$, diradical 2 in chloroform-d (label: SM-11-20-R2). 

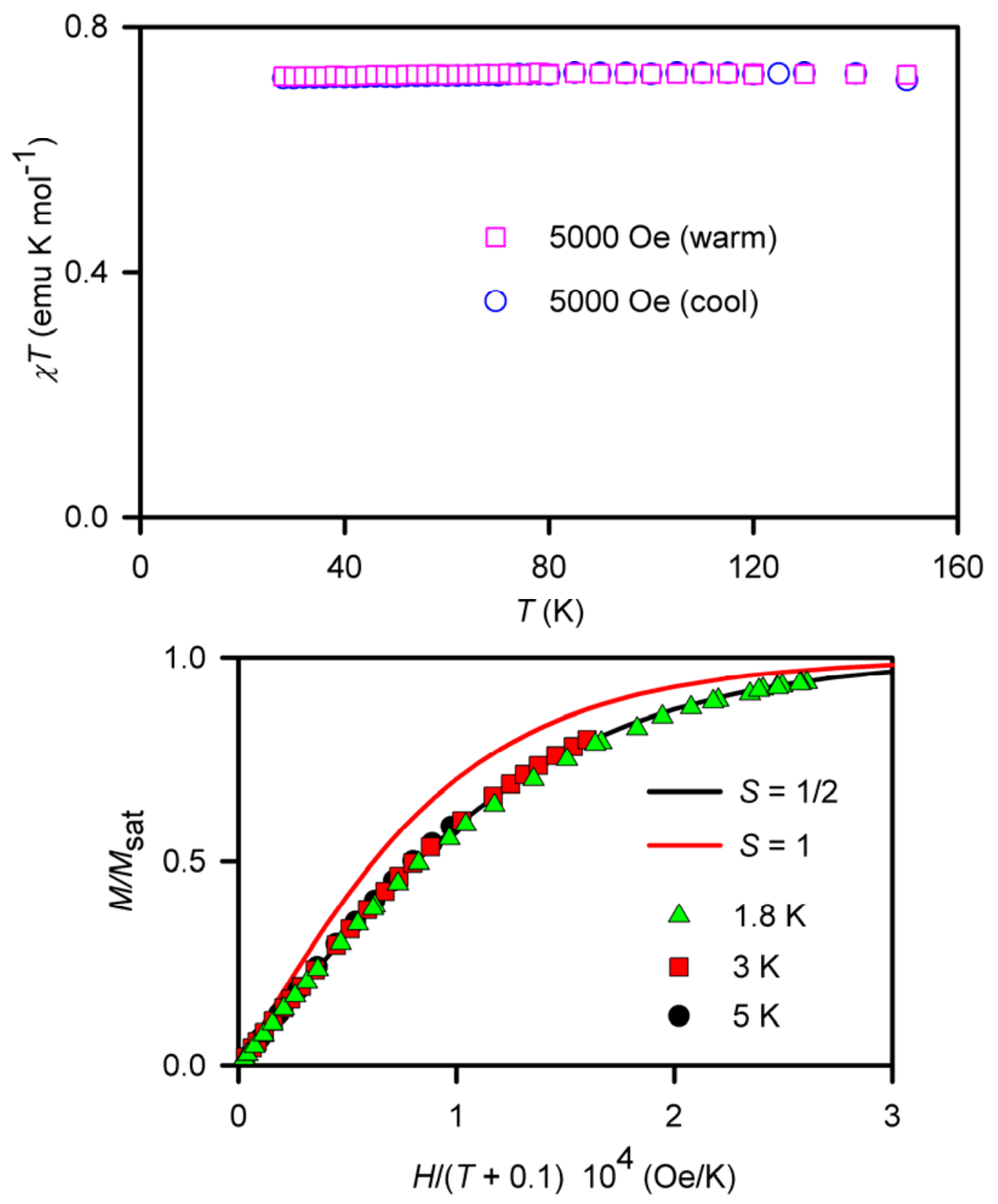

Figure S6. SQUID magnetometry for $\sim 20 \mathrm{mM}$ nitronyl nitroxide diradical 2 in chloroform (label: $\mathrm{SM} 10100 \mathrm{H})$. Top plot corresponds to $\chi T$ vs. $T$ in both cooling and warming modes, with $\chi T=0.72$ emu $\mathrm{K} \mathrm{mol}^{-1}$ in the high temperature range. Bottom plot corresponds to $M / M_{\text {sat }} \mathrm{vs} . H /(T-\theta)$, with solid lines showing plots of Brillouin functions with $S=1 / 2$ and $S=1$; numerical fits to the Brillouin functions with $\theta \approx-0.1 \mathrm{~K}$ give $S \approx 0.5$ and $M_{\text {sat }}=0.94 \mu_{\mathrm{B}}$ at $1.8,3$, and $5 \mathrm{~K}$. 


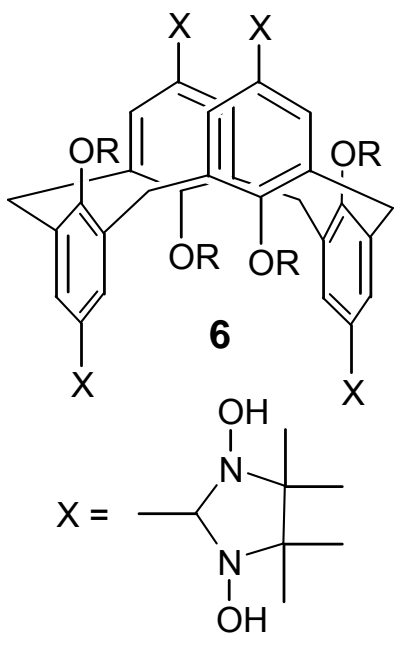

$$
\mathrm{R}=\mathrm{CH}_{2} \mathrm{CH}_{2} \mathrm{OMe}
$$

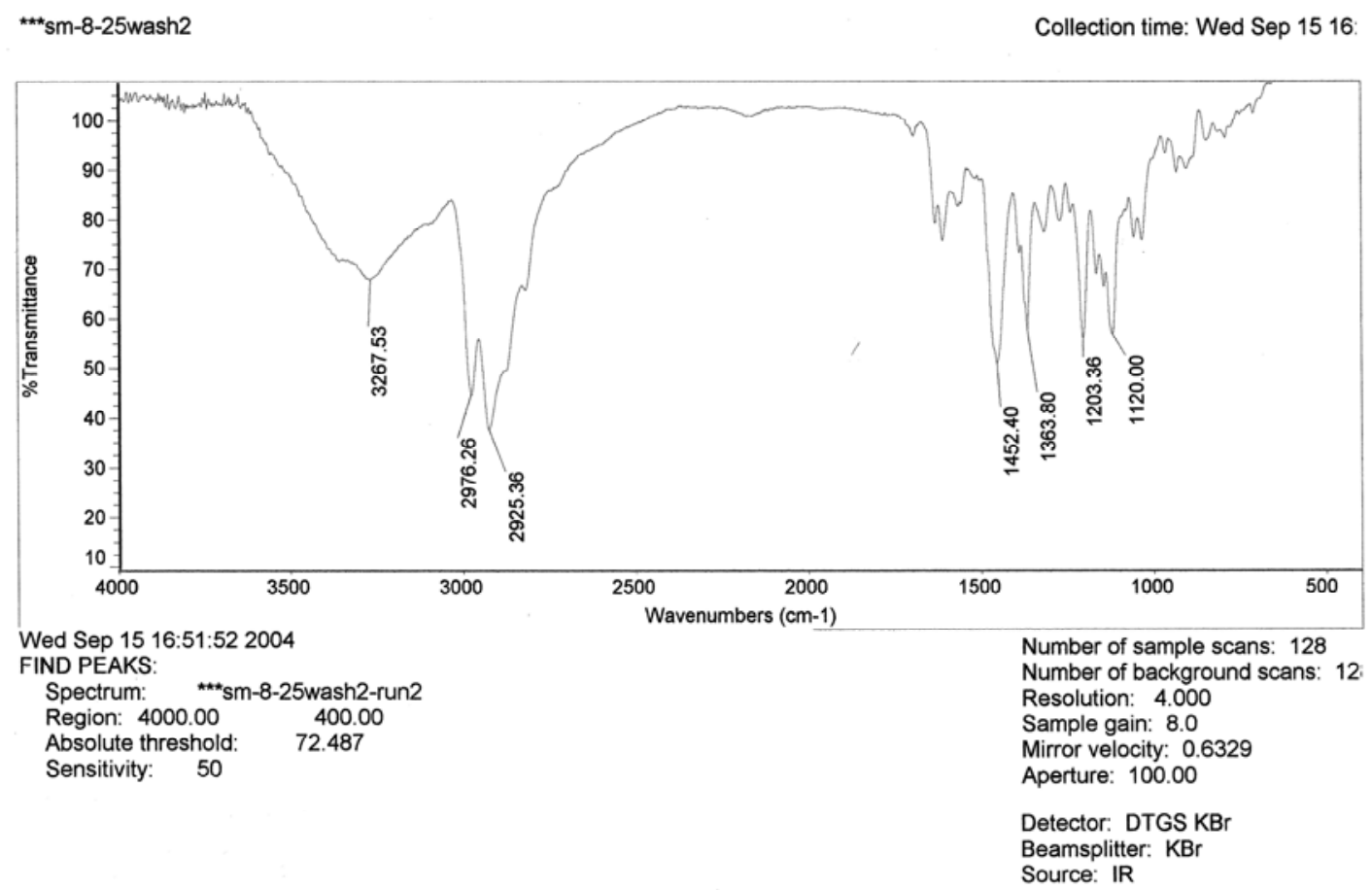

Figure S7. IR (ATR, ZnSe) spectrum of 5,11,17,23-tetrakis(1,3-dihydroxy-4,4,5,5tetramethylimidazolin-2-yl)-25,26,27,28-tetrakis(methoxyethoxy)calix[4]arene 6 (sm-8-25wash2). 

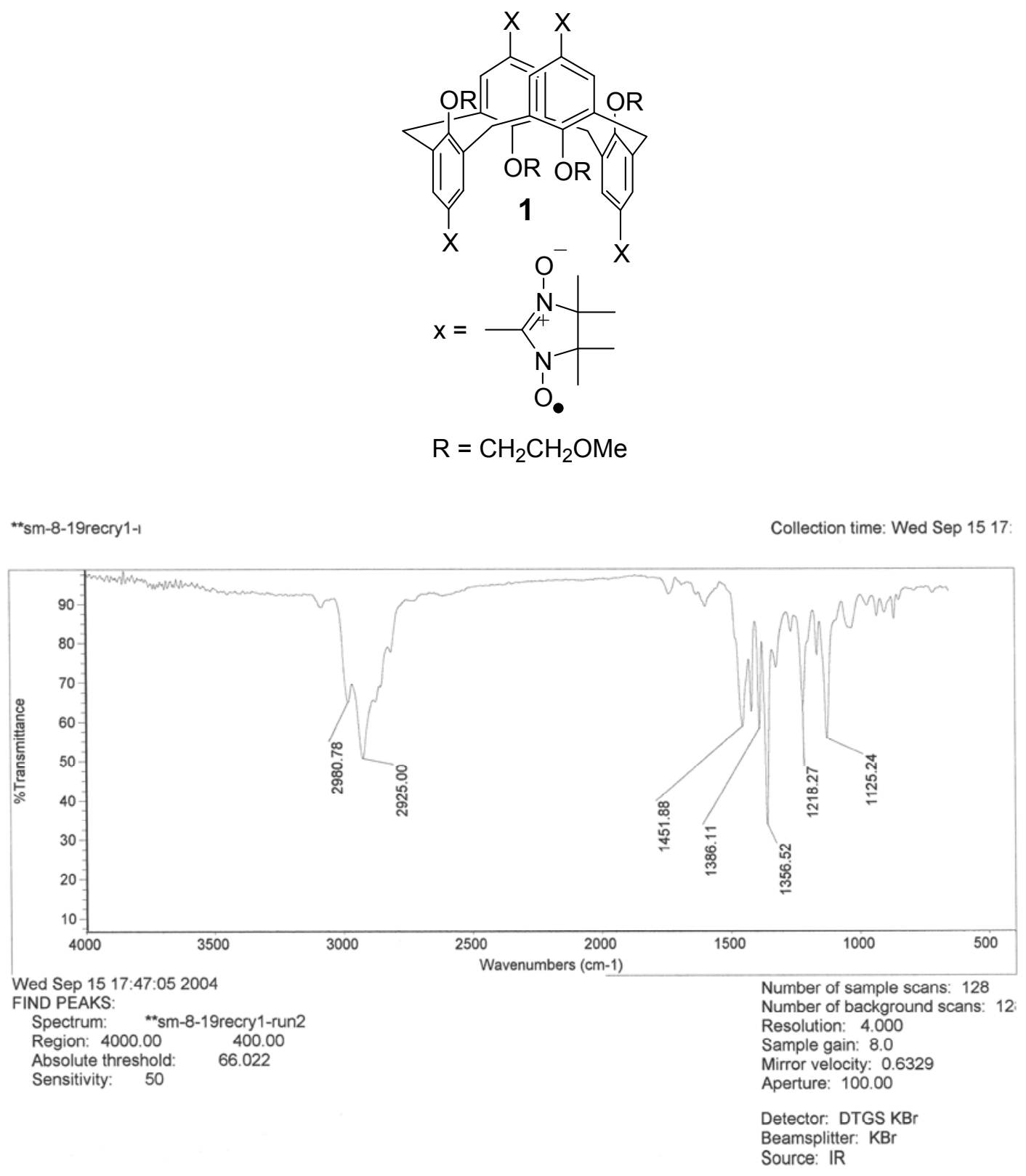

Figure S8. IR (ATR, ZnSe) spectrum of 5,11,17,23-tetrakis(1-oxyl-3-oxo-4,4,5,5tetramethylimidazolin-2-yl)-25,26,27,28-tetrakis(methoxyethoxy)calix[4]arene 1 (sm-8-19recry1-run2). 

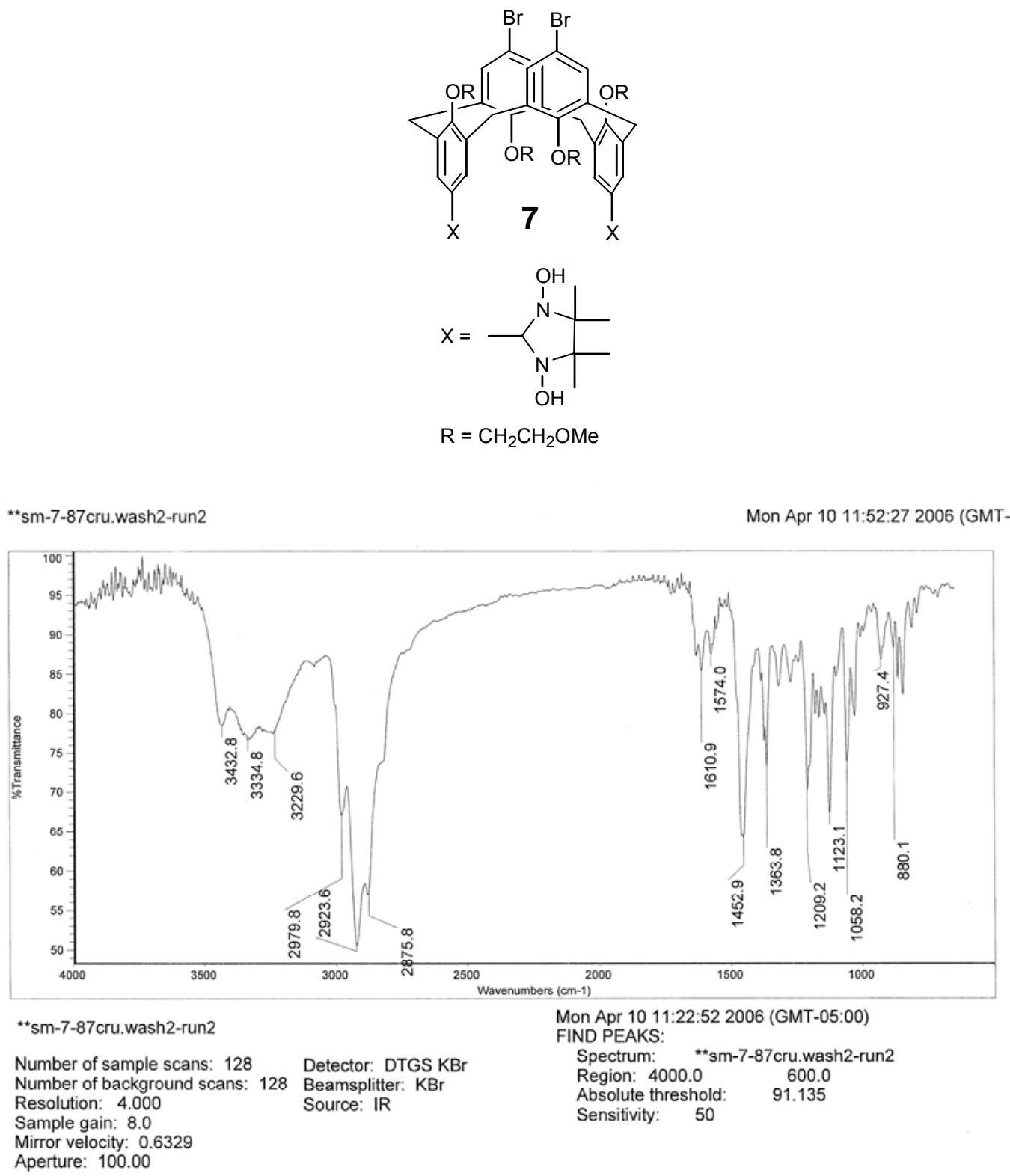

Figure S9. IR (ATR, ZnSe) spectrum of 5,17-bis(1,3-dihydroxy-4,4,5,5-tetramethylimidazolin-2-yl)11,23-dibromo-25,26,27,28-tetrakis(methoxyethoxy)-calix[4]arene 7 (sm-7-87wash1). 


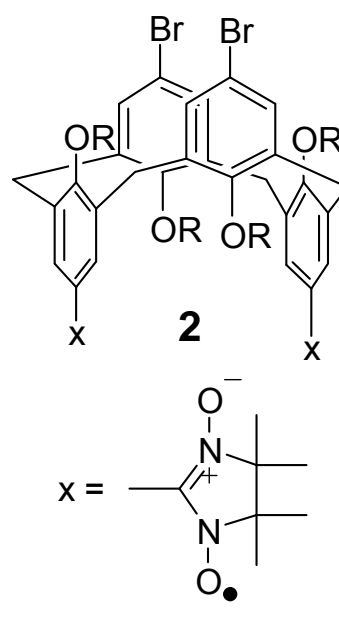

$\mathrm{R}=\mathrm{CH}_{2} \mathrm{CH}_{2} \mathrm{OMe}$

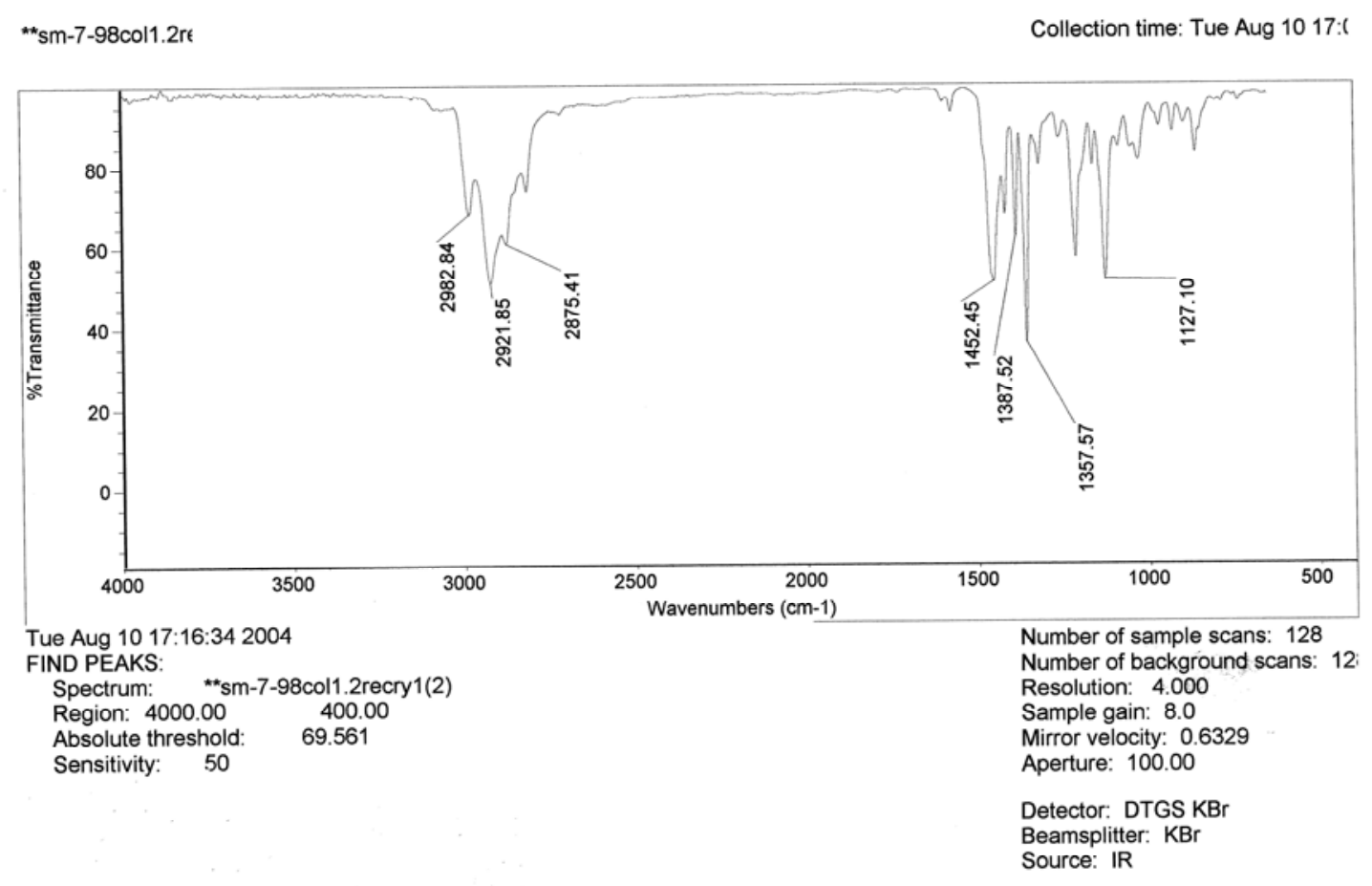

Figure S10. IR (ATR, ZnSe) spectrum of 5,17-bis(1-oxyl-3-oxo-4,4,5,5-tetramethylimidazolin-2-yl)11,23-dibromo-25,26,27,28-tetrakis(methoxyethoxy)-calix[4]arene 2 (sm-7-98col1.2recry1(2)). 


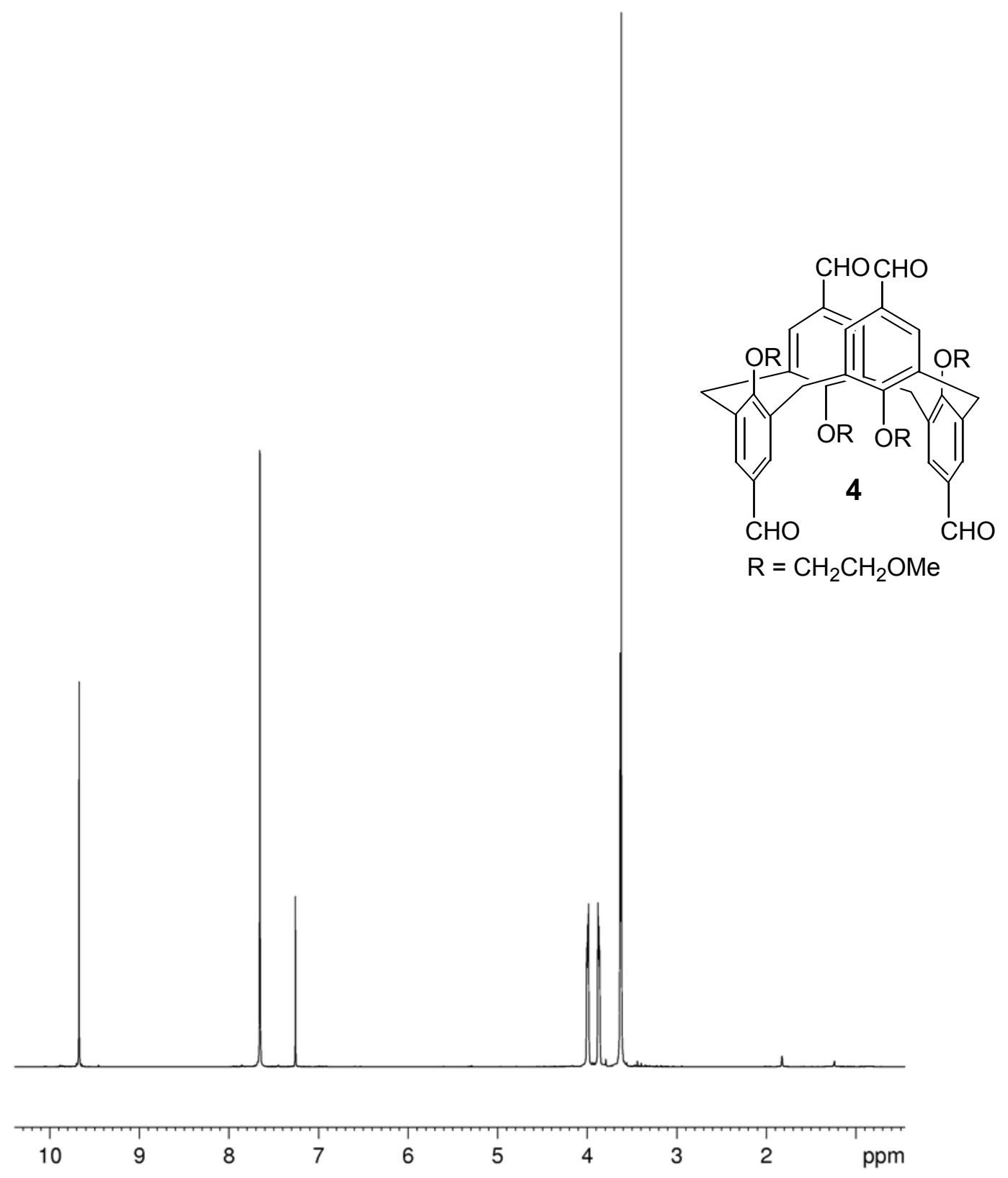

Figure S11. ${ }^{1} \mathrm{H} \quad \mathrm{NMR}\left(400 \mathrm{MHz}, \mathrm{CDCl}_{3}\right)$ spectrum of 5,11,17,23-tetraformyl-25,26,27,28tetrakis(methoxyethoxy)calix[4]arene 4 (sm-10-wash1). 

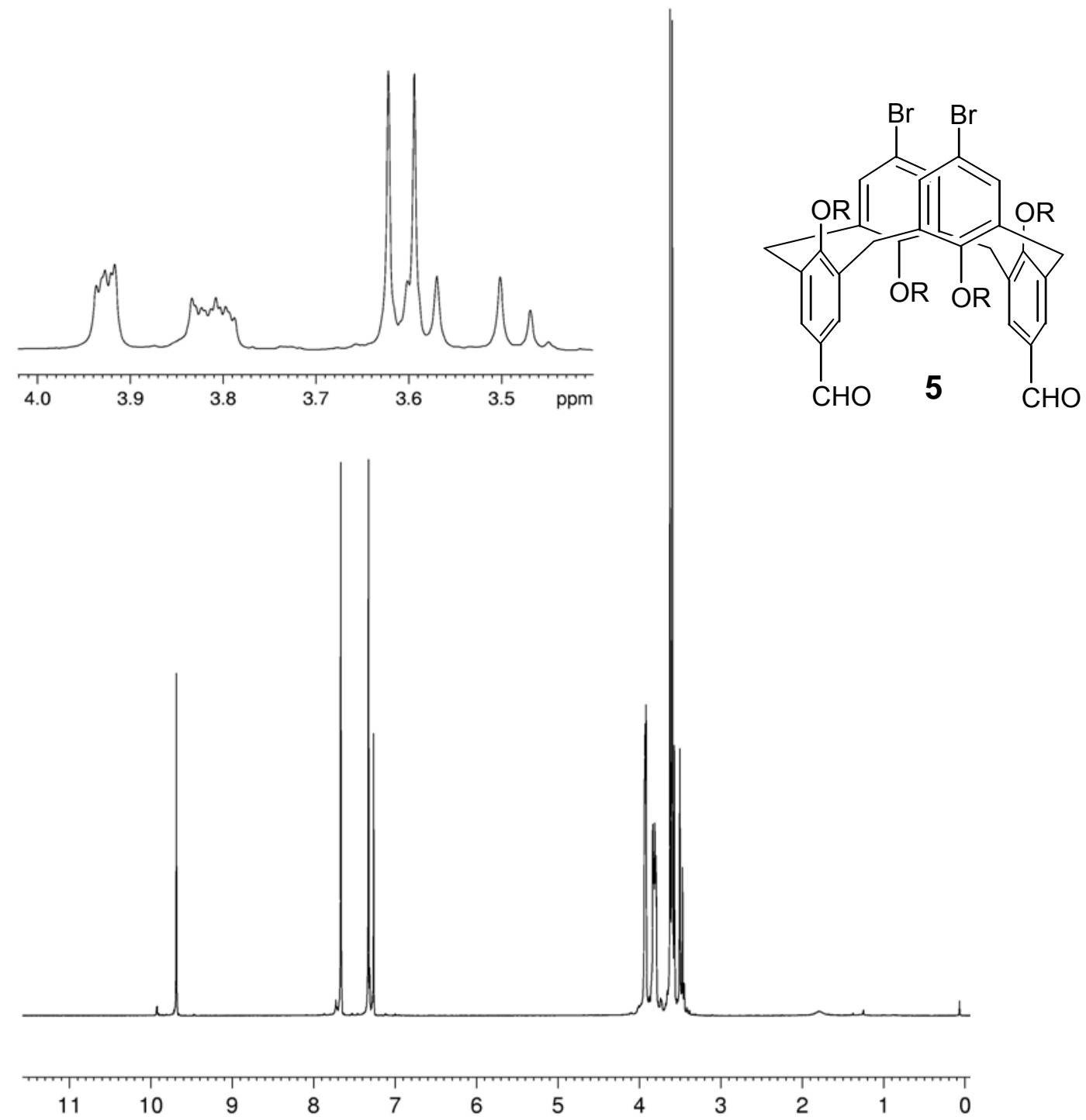

Figure S12. ${ }^{1} \mathrm{H}$ NMR (400 $\mathrm{MHz}, \mathrm{CDCl}_{3}$ ) spectrum of 5,17-diformyl-11,23-dibromo-25,26,27,28tetrakis(methoxyethoxy)calix[4]arene 5 (sm-6-79recry2_run2). 


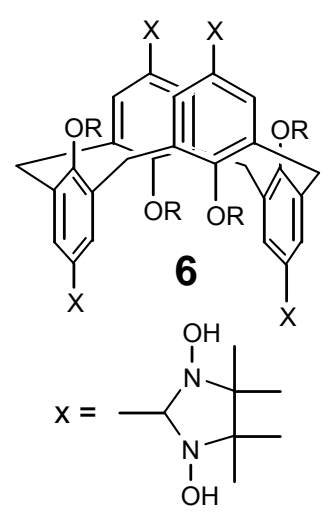

$\mathrm{R}=\mathrm{CH}_{2} \mathrm{CH}_{2} \mathrm{OMe}$
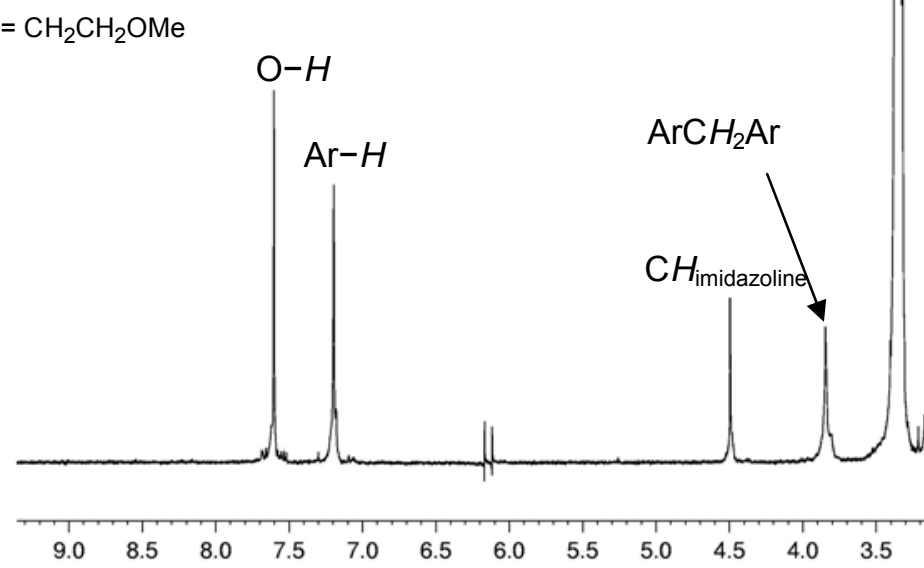
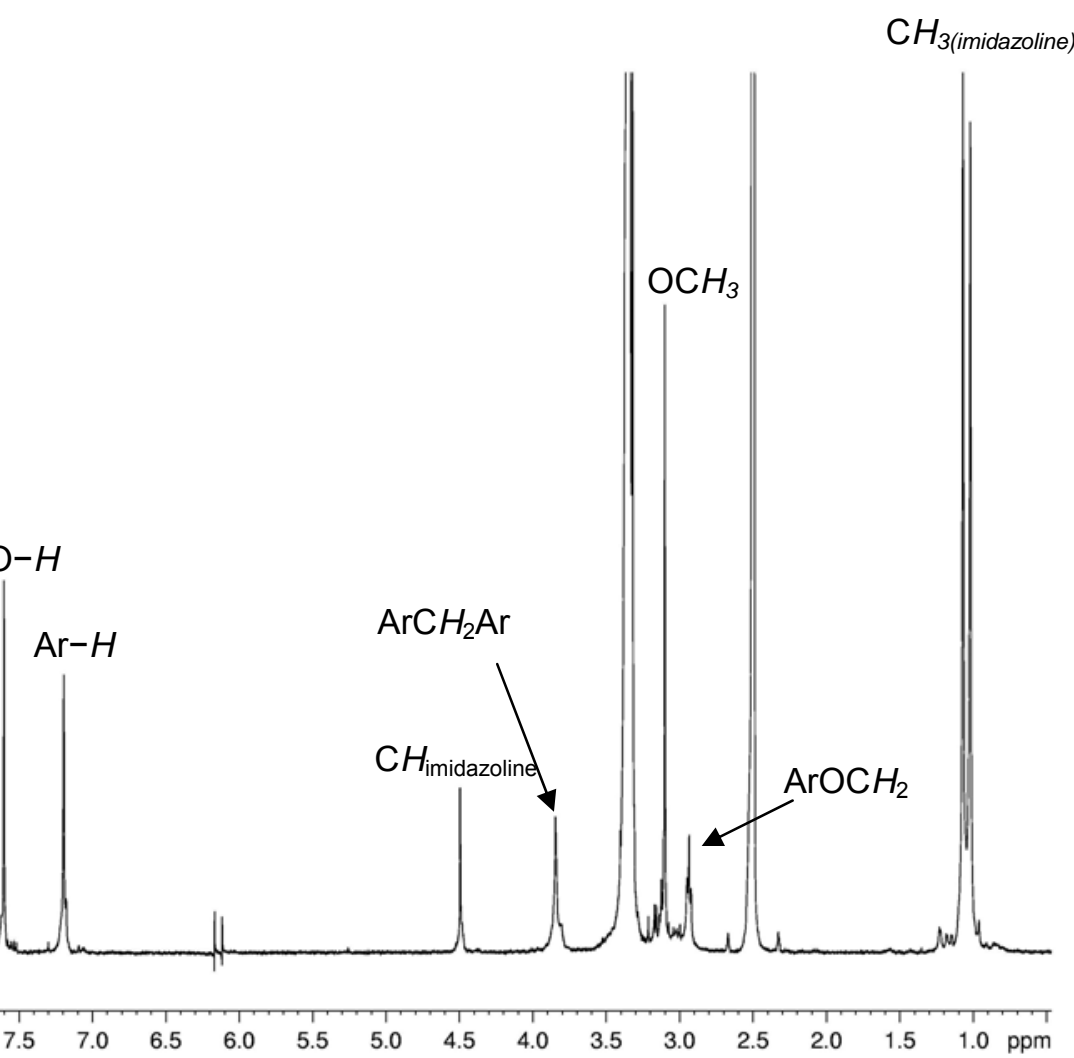

Figure S13. ${ }^{1} \mathrm{H}$ NMR (400 MHz, DMSO- $d_{6}$ ) spectrum for the 5,11,17,23-tetrakis(1,3-dihydroxy4,4,5,5-tetramethylimidazolin-2-yl)-25,26,27,28-tetrakis(methoxyethoxy)-calix[4]arene 6 (sm-8-28cru). 


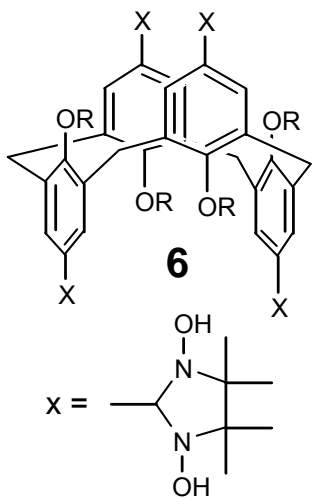

$\mathrm{R}=\mathrm{CH}_{2} \mathrm{CH}_{2} \mathrm{OMe}$

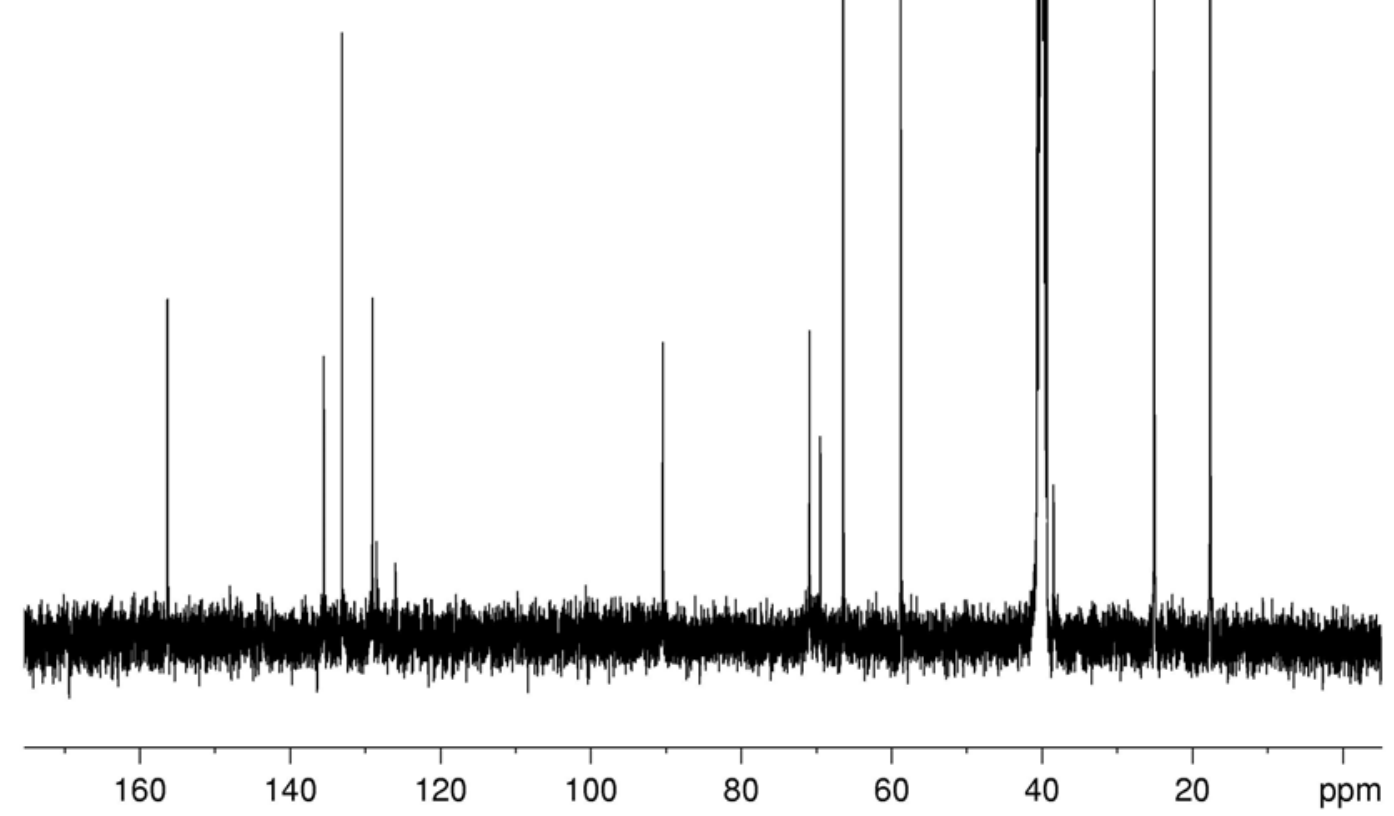

Figure S14. ${ }^{13} \mathrm{C}\left\{{ }^{1} \mathrm{H}\right\}$ NMR (100 MHz, DMSO- $\left.d_{6}\right)$ spectrum of 5,11,17,23-tetrakis(1,3-dihydroxy4,4,5,5-tetramethylimidazolin-2-yl)-25,26,27,28-tetrakis(methoxyethoxy)-calix[4]arene $6 \quad$ (sm-1085wash1_C13). 

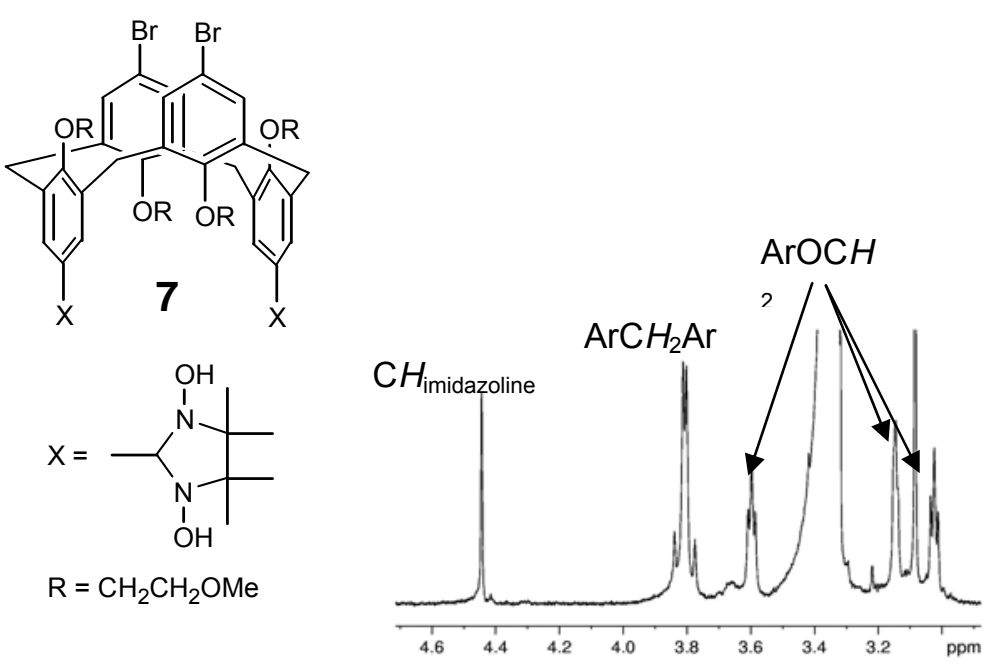

$\mathrm{O}-\mathrm{H}$

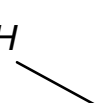

\section{$\mathrm{ArH}$}<smiles></smiles>
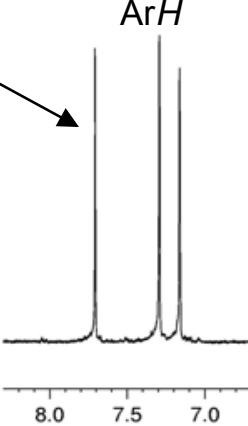

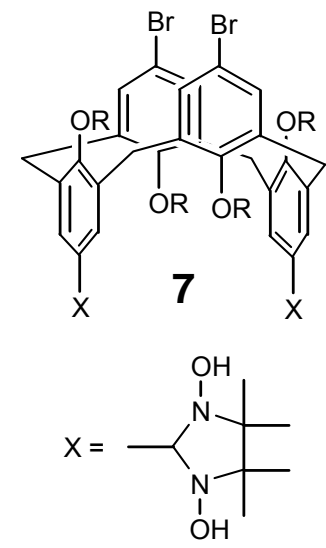

$\mathrm{R}=\mathrm{CH}_{2} \mathrm{CH}_{2} \mathrm{OMe}$
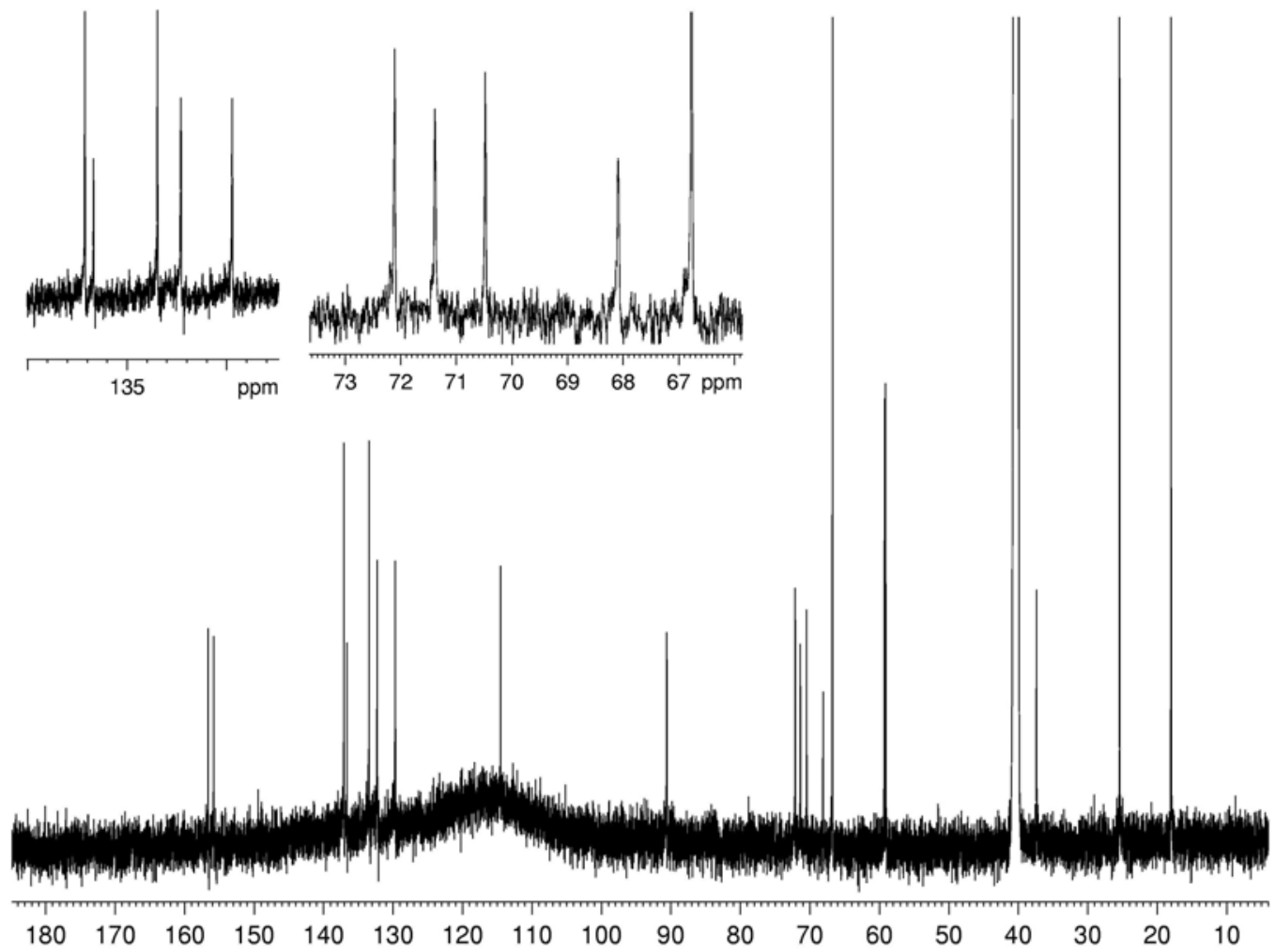

Figure S16. ${ }^{13} \mathrm{C}\left\{{ }^{1} \mathrm{H}\right\}$ NMR (150 MHz, DMSO-d $\left.d_{6}\right)$ spectrum of 5,17-bis(1,3-dihydroxy-4,4,5,5tetramethylimidazolin-2-yl)-11,23-dibromo-25,26,27,28-tetrakis(methoxyethoxy) calix[4]arene 7 (sm7-87wash1.C13). 


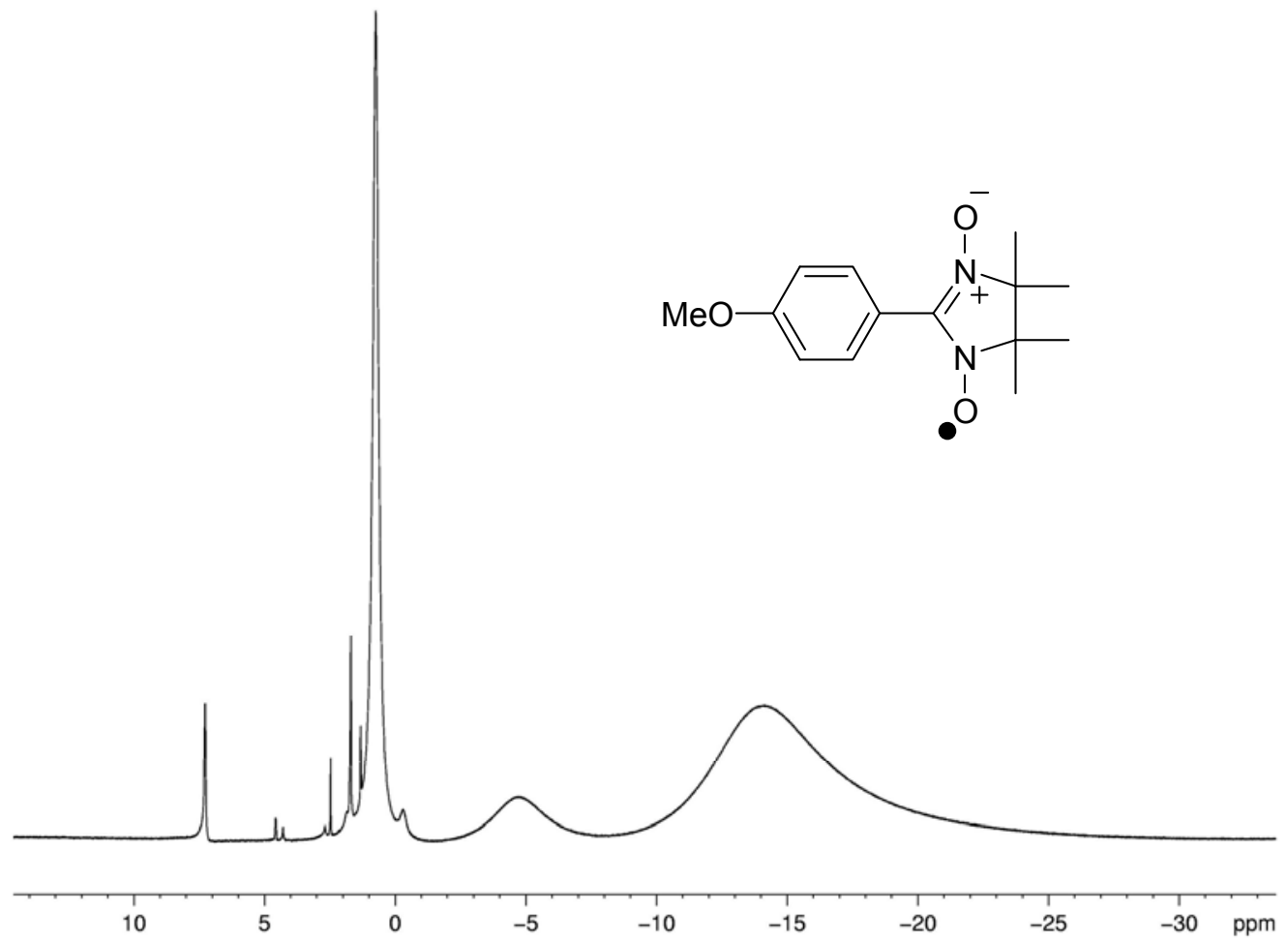

Figure S17. ${ }^{1} \mathrm{H}$ NMR (400 MHz, DMSO-d $)$ spectrum of 2-(4-methoxyphenyl-4,4,5,5tetramethylimidazoline-1-oxyl-3-oxide (sm-7-58anisrecry1). 


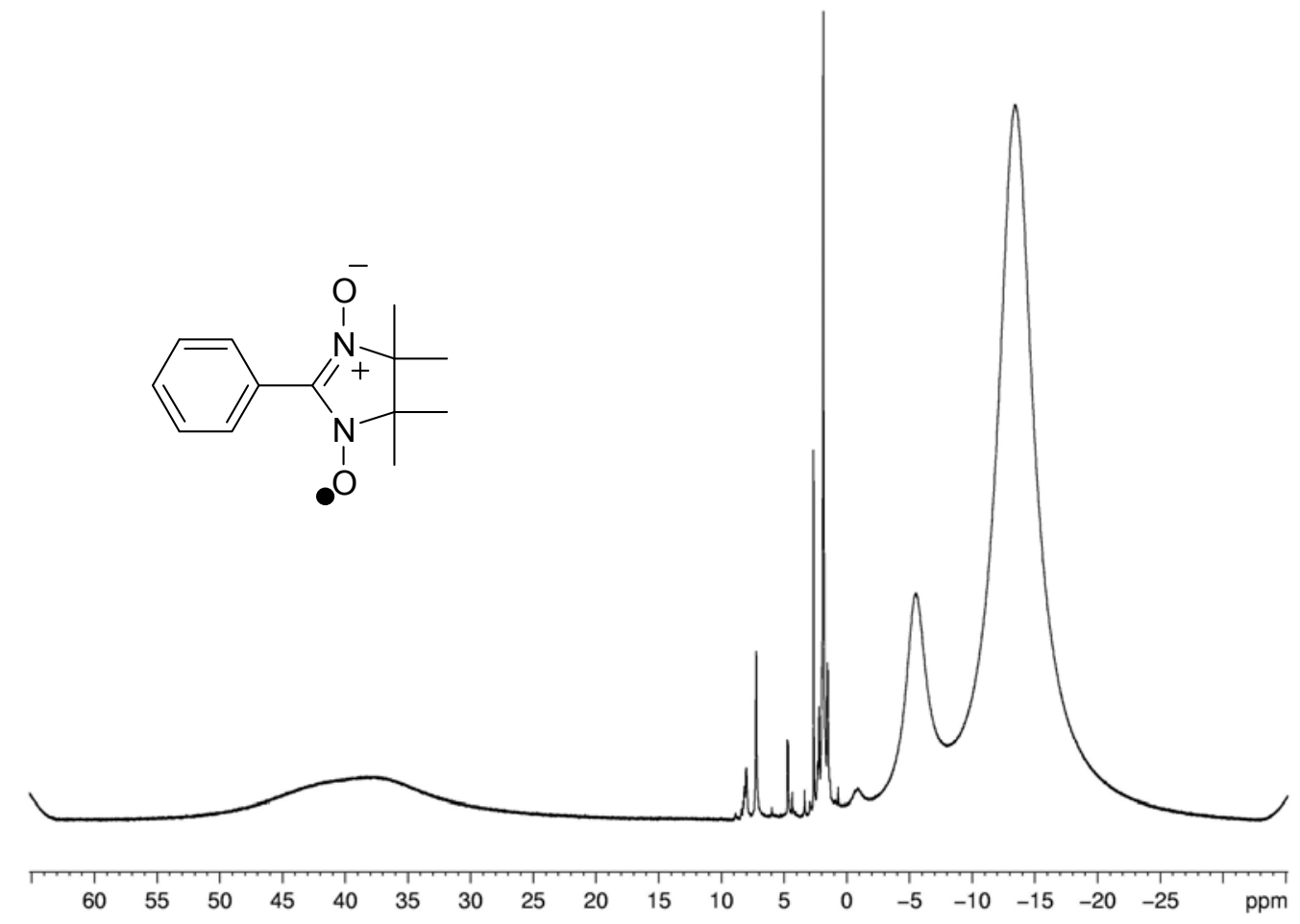

Figure S18. ${ }^{1} \mathrm{H}$ NMR (400 MHz, DMSO- $\left.d_{6}\right)$ spectrum of 2-phenyl-4,4,5,5-tetramethylimidazoline-1oxyl-3-oxide (sm-7-58benzal-N,Ncol1). 